BULLETIN (New Series) OF THE

AMERICAN MATHEMATICAL SOCIETY

Volume 51, Number 1, January 2014, Pages 71-130

S 0273-0979(2013)01421-6

Article electronically published on July 15, 2013

\title{
LINEAR SUBSETS OF NONLINEAR SETS IN TOPOLOGICAL VECTOR SPACES
}

\author{
LUIS BERNAL-GONZÁLEZ, DANIEL PELLEGRINO, AND JUAN B. SEOANE-SEPÚLVEDA
}

\begin{abstract}
For the last decade there has been a generalized trend in mathematics on the search for large algebraic structures (linear spaces, closed subspaces, or infinitely generated algebras) composed of mathematical objects enjoying certain special properties. This trend has caught the eye of many researchers and has also had a remarkable influence in real and complex analysis, operator theory, summability theory, polynomials in Banach spaces, hypercyclicity and chaos, and general functional analysis. This expository paper is devoted to providing an account on the advances and on the state of the art of this trend, nowadays known as lineability and spaceability.
\end{abstract}

\section{Contents}

1. Introduction. "Strange" mathematical objects throughout history

2. Special subsets of real and complex functions

2.1. Continuous nowhere differentiable functions (Weierstrass' Monsters)

2.2. Differentiable functions and related properties

2.3. Continuous (and nowhere continuous) functions

2.4. Measurability and integration

2.5. Series and summability

2.6. Nonextendable holomorphic functions

2.7. Miscellaneous

3. Hypercyclic manifolds

3.1. Hypercyclicity and universality: examples and genericity

3.2. Hypercyclicity and lineability

3.3. Other kinds of linear chaos

4. Zeros of polynomials in Banach spaces

5. Some remarks and conclusions. General techniques

About the authors

Acknowledgments

References

Received by the editors November 26, 2012, and, in revised form, April 2, 2013.

2010 Mathematics Subject Classification. Primary 15A03, 46E10, 46E15; Secondary 26B05, 28A20, 47A16, 47L05.

Key words and phrases. Lineability, spaceability, algebrability, real and complex analysis, special functions, operator theory, Baire category theorem, hypercyclic manifolds, zeros of polynomials.

The first author was partially supported by Ministerio de Economía y Competitividad Grant MTM2012-34847-C02-01.

The second author was supported by INCT-Matemática, CAPES-NF, CNPq Grants 301237/2009-3 and 477124/2012-7.

The third author was supported by MTM2012-34341. 


\section{INTRODUCTION. \\ "Strange" MATHEMATiCAL OBJECTS THROUGHOUt History}

Throughout history there have always been mathematical objects that have contradicted the intuition of the working mathematician. To cite some of these objects, let us recall the famous Weierstrass' Monster, Sierpiński's carpet, discontinuous additive functions (or Jones' functions), Peano curves, Cantor functions, or even the more modern differentiable nowhere monotone functions.

One may think that, once such an object is found, not many more like it can possibly exist. History has proven this last statement wrong. It is actually so wrong that, at the present time, the appearance of these exotic mathematical objects no longer comes as a surprise to mathematicians. (For a quite complete account of some of these so-called strange objects we refer the interested reader to the monographs 161, 163, 198, 263, 273.)

A classical, famous, and very powerful technique that one can use to obtain some kinds of unexpected objects is the very famous Baire category theorem. One version of this theorem states that, in any complete metric space $E$, the complement of a first category subset of $E$ is everywhere dense in $E$. It usually happens that this complement consists exactly of strange (in certain sense) elements; for instance, the Baire category theorem can be applied to show that "most" real valued $C^{\infty}$. functions are nowhere analytic, as seen in Section 2.2.2. Unfortunately, the Baire category theorem does not apply to many cases, and sometimes one needs to employ a constructive approach when tackling certain problems.

For the last decade there has been a generalized trend in mathematics toward the search for large algebraic structures of special objects (and sometimes called "pathological" in the literature [156, 253]). Let us introduce some terminology before carrying on. A subset $M$ of a topological vector space $X$ is called lineable (resp. spaceable) in $X$ if there exists an infinite dimensional linear space (resp. an infinite dimensional closed linear space) $Y \subset M \cup\{0\}$. These notions of lineable and spaceable were originally coined by Vladimir I. Gurariy, and they first appeared in 22, 181,253. Prior to the publication of these previous works, some authors, when working with infinite dimensional spaces, already found large linear structures enjoying these types of special properties, even though they did not explicitly use terms like lineability or spaceability; see, e.g., 19. One of the first results illustrating this was due to Levine and Milman.

Theorem 1.1 (Levine and Milman, 1940 [211]). The subset of $\mathcal{C}[0,1]$ of all continuous functions on $[0,1]$ of bounded variation is not spaceable.

As usual, by $\mathcal{C}[0,1]$ we have denoted the Banach space of continuous functions $[0,1] \rightarrow \mathbb{R}$ endowed with the supremum norm. Later, the following famous result on the set of continuous nowhere differentiable functions was proved by Gurariy.

Theorem 1.2 (Gurariy, 1966 [178]). The set of continuous nowhere differentiable functions on $[0,1]$ is lineable.

Let us also recall that, although the set of everywhere differentiable functions in $\mathbb{R}$ is, in itself, an infinite dimensional vector space, in 1966 Gurariy obtained the following analogue of Theorem 1.1 . 
Theorem 1.3 (Gurariy, 1966 [178]). The set of everywhere differentiable functions on $[0,1]$ is not spaceable in $\mathcal{C}[0,1]$. Also, there exist closed infinite dimensional subspaces of $\mathcal{C}[0,1]$ all of whose members are differentiable on $(0,1)$.

Somehow, what we are seeing is that what one could expect to be an isolated phenomenon can actually even have a nice algebraic structure (in the form of infinite dimensional subspaces). Unfortunately, and as we mentioned above, the Baire category theorem cannot be employed in the search for large subspaces such as the ones mentioned in the previous results. Let us now provide a more formal and complete definition for the above concepts and some other ones.

Definition 1.4 (Lineability and spaceability [22,181,253]). Let $X$ be a topological vector space and $M$ a subset of $X$. Let $\mu$ be a cardinal number.

(1) $M$ is said to be $\mu$-lineable ( $\mu$-spaceable) if $M \cup\{0\}$ contains a vector space (resp. a closed vector space) of dimension $\mu$. At times, we shall refer to the set $M$ as simply lineable or spaceable if the existing subspace is infinite dimensional.

(2) We also let $\lambda(M)$ be the maximum cardinality (if it exists) of such a vector space 1

(3) When the above linear space can be chosen to be dense in $X$, we shall say that $M$ is $\mu$-dense-lineable.

Moreover, Bernal introduced in 69 the notion of maximal-lineable (and those of maximal-dense-lineable and maximal-spaceable), meaning that, when keeping the above notation, the dimension of the existing linear space equals $\operatorname{dim}(X)$. Besides asking for linear spaces, one could also study other structures, such as algebrability and some related ones, which were presented in [25, 27, 253.

Definition 1.5. Given a Banach algebra $\mathcal{A}$, a subset $\mathcal{B} \subset \mathcal{A}$, and two cardinal numbers $\alpha$ and $\beta$, we say that:

(1) $\mathcal{B}$ is algebrable if there is a subalgebra $\mathcal{C}$ of $\mathcal{A}$ such that $\mathcal{C} \subset \mathcal{B} \cup\{0\}$ and the cardinality of any system of generators of $\mathcal{C}$ is infinite.

(2) $\mathcal{B}$ is dense-algebrable if, in addition, $\mathcal{C}$ can be taken dense in $\mathcal{A}$.

(3) $\mathcal{B}$ is $(\alpha, \beta)$-algebrable if there is an algebra $\mathcal{B}_{1}$ such that $\mathcal{B}_{1} \subset \mathcal{B} \cup\{0\}$, $\operatorname{dim}\left(\mathcal{B}_{1}\right)=\alpha, \operatorname{card}(S)=\beta$, and $S$ is a minimal system of generators of $\mathcal{B}_{1} 2$

(4) At times we shall say that $\mathcal{B}$ is, simply, $\kappa$-algebrable if there exists a $\kappa$ generated subalgebra $\mathcal{C}$ of $\mathcal{A}$ with $\mathcal{C} \subset \mathcal{B} \cup\{0\}$.

\footnotetext{
${ }^{1}$ Indeed, this $\lambda(M)$ might not exist. It is not difficult to provide natural examples of sets which are $n$-lineable for every $n \in \mathbb{N}$ but which are not infinitely lineable. For instance, let $j_{1} \leq k_{1}<j_{2} \leq \cdots \leq k_{m}<j_{m+1} \leq \cdots$ be integers, and let $M=\bigcup_{m}\left\{\sum_{i=j_{m}}^{k_{m}} a_{i} x^{i}: a_{i} \in \mathbb{R}\right\}$. Since the sets $\left\{\sum_{i=j_{m}}^{k_{m}} a_{i} x^{i}: a_{i} \in \mathbb{R}\right\}(m \in \mathbb{N})$ are pairwise disjoint, $M$ is finitely, but not infinitely, lineable in $\mathcal{C}[0,1]$. Depending on the choice of the sequence $\left(j_{n}\right), M$ may even be closed in $\mathcal{C}[0,1]$. For instance, it is shown in [180] that if $\left(j_{n}\right)$ is a lacunary sequence, then $\left\{x_{n}^{j_{n}}\right\}_{n \geq 1}$ is a basic sequence in $\mathcal{C}[0,1]$. On the other hand, no matter what sequence $j_{1} \leq k_{1}<j_{2} \leq \cdots$ we take, the corresponding set of complex polynomials $M=\bigcup_{n}\left\{\sum_{\ell=j_{n}}^{k_{n}} a_{\ell} z^{\ell}: a_{\ell} \in \mathbb{C}\right\}$ is always closed in $H^{\infty}$, the Banach space of holomorphic functions in the open unit disc of $\mathbb{C}$ endowed with the supremum norm.

${ }^{2}$ Here, by " $S$ is a minimal set of generators of an algebra $\mathcal{D}$ " we mean that $\mathcal{D}=\mathcal{A}(S)$ is the algebra generated by $S$, and for every $x_{0} \in S, x_{0} \notin \mathcal{A}\left(S \backslash\left\{x_{0}\right\}\right)$.
} 
We also say that a subset $M$ of a linear algebra $\mathcal{L}$ is strongly $\kappa$-algebrable if there exists a $\kappa$-generated free algebra $\mathcal{A}$ contained in $M \cup\{0\}$ (see 39]). Other types of structures have also been considered, such as cones 3 or modules 4 The links between the previous concepts are as follows:

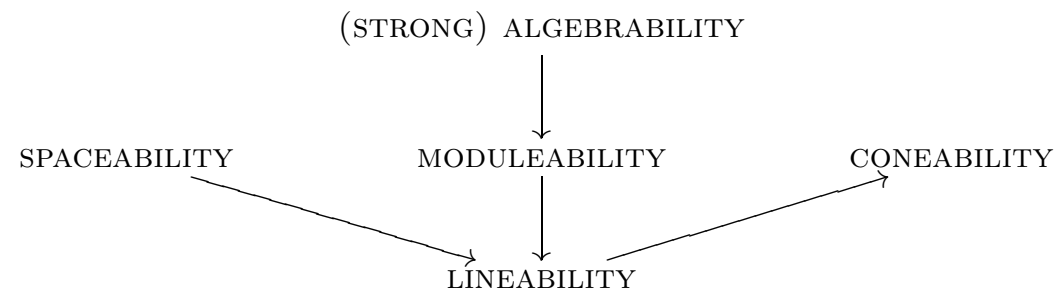

All the implications in the previous diagram are strict. Specifically, examples of sets that are lineable but not spaceable, coneable but not lineable, lineable but not moduleable, moduleable but not algebrable, and algebrable but not strongly algebrable, can be found, respectively, in [178, [2], [157], and [40].

In this survey paper, we will mainly focus on subspaces and subalgebras in topological vector spaces. The paper is arranged in four main sections, dealing with lineability, spaceability, and algebrability within many different frameworks. The text is organized as follows:

Section 2. In this part, we focus on real and complex valued functions. In connection with the existence of strange objects, classical mathematical analysis in real and complex variables is certainly the largest existing source.

Section 3. The theory of hypercyclic operators has experienced an exponential development since its birth at the end of the twentieth century, and it deserves a separate section itself. The construction of infinite algebraic structures of hypercyclic vectors is still an ongoing problem nowadays studied by many authors.

Section 4. The topic of polynomials in Banach spaces and their sets of zeros also deserves a special place in this survey since it has recently been linked to this theory of lineability and spaceability. This topic has just recently started to expand, it has rapidly attracted the interest of many researchers in the area, and there are still several ongoing works on it.

Section 5. In the last part we shall make some remarks on lineability and several open problems in functional analysis. Moreover, we provide an account on all the existing techniques that can be applied to general situations in different frameworks in order to achieve lineability or even dense-lineability, and spaceability.

Throughout this survey, open problems will be given and directions will be also indicated to tackle new problems in this theory. We believe that the questions and open problems found here are of interest in many areas of mathematics, and their answers (either in the positive or in the negative) would certainly help to develop the recently coined theories of lineability and spaceability.

\footnotetext{
${ }^{3}$ A set of functions in $\mathbb{R}^{\mathbb{R}}$ (or $\mathbb{C}^{\mathbb{C}}$ ) is said to be coneable if it possesses a positive (or negative) cone containing an infinite linearly independent set; see 2, Definition 1.1].

${ }^{4}$ Let $L$ be a subset of a Banach algebra (or a topological algebra) $X$. We say that $L$ is moduleable if there exists an infinitely generated subalgebra $M$ of $X$ and an infinitely generated additive subgroup $G$ of $X$ such that $G$ is an $(M, \mathbb{K})$-bimodule, $G$ is $\mathbb{K}$ infinite dimensional, and $L \cup\{0\} \supset G$. For more information on this notion, we refer to [157. Definition 1.2].
} 
Standard symbols and notation that will be used throughout the paper include the following. The symbols, $\mathbb{N}, \mathbb{N}_{0}, \mathbb{Z}, \mathbb{Q}, \mathbb{R}$, and $\mathbb{C}$ denote, respectively, the set of positive integers, the set $\mathbb{N} \cup\{0\}$, the ring of integers, the field of rational numbers, the real line, and the complex plane. The symbols $\aleph_{0}$ and $\mathfrak{c}$ stand for the cardinality of $\mathbb{N}$ and the cardinality of the continuum, respectively. A subset $A$ of a topological space $X$ is called $G_{\delta}$ (resp. $F_{\sigma}$ ) if $A$ is a countable intersection of dense open subsets (resp. a countable union of closed subsets each of them with empty interior). Hence $A$ is a $G_{\delta}$ set if and only if its complement is an $F_{\sigma}$ set. Moreover, a subset $A \subset X$ is said to be residual provided that its complement $X \backslash A$ is of the first category; that is, $X \backslash A$ can be written as a countable union of sets whose closures have empty interiors. Therefore, by Baire's category theorem, residual subsets are topologically large in a complete metric space.

\section{Special Subsets of REAL AND COMPlex FUnCtions}

\subsection{Continuous nowhere differentiable functions (Weierstrass' Mon-}

sters). It came as a general shock when, in 1872 and during a presentation before the Berlin Academy, K. Weierstrass provided a classical example of a function that was continuous everywhere but differentiable nowhere. The particular example was defined as

$$
f(x)=\sum_{k=0}^{\infty} a^{k} \cos \left(b^{k} \pi x\right),
$$

where $0<a<1, b$ is any odd integer, and $a b>1+3 \pi / 2$.

The apparent shock was a consequence of the general thought that most mathematicians shared: that a continuous function must have derivatives at a significant set of points; even A. M. Ampère attempted to give a theoretical justification for this. Although the first published example is certainly due to Weierstrass, already in 1830 the Czech mathematician B. Bolzano exhibited a continuous nowhere differentiable function. Let us give a brief overview of the appearance throughout history of "Weierstrass' Monsters" (see, e.g., 264 for a thorough study of the citations below):

\begin{tabular}{l|r}
\hline Discoverer & Year \\
\hline B. Bolzano & $\approx 1830$ \\
M. Ch. Cellérier & $\approx 1830$ \\
B. Riemann & 1861 \\
H. Hankel & 1870 \\
K. Weierstrass & 1872
\end{tabular}

After 1872 many other mathematicians also constructed similar functions. Just to cite a partial list of these, we have: H. A. Schwarz (1873), M. G. Darboux (1874), U. Dini (1877), K. Hertz (1879), G. Peano (1890), D. Hilbert (1891), T. Takagi (1903), H. von Koch (1904), W. Sierpiński (1912), G. H. Hardy (1916), A. S. Besicovitch (1924), B. van der Waerden (1930), S. Mazurkiewicz (1931), S. Banach (1931), S. Saks (1932), and W. Orlicz (1947). We would also like to refer the interested reader to [7,191] for some recent results on this class of continuous nowhere differentiable functions. 
As a nice application of the Baire category theorem, Banach obtained in 1931 that most continuous functions are nowhere differentiable; see, e.g., 235. Specifically, the set of all continuous but nowhere differentiable functions on $\mathbb{R}$ is residual in $\mathcal{C}(\mathbb{R})$, when endowed with the topology of uniform convergence in compacta.

Later, at the end of the twentieth century and nowadays, there are also authors who have, as well, constructed Weierstrass' Monsters with even additional "pathologies". The lineability of this type of functions has been thoroughly studied in recent years. The very first result in this direction was due to V. I. Gurariy in 1966 (178, 179] and Theorem 1.2 in Section 1), who showed that the set of continuous nowhere differentiable functions on $[0,1]$ is lineable. The lineability of this class of functions has been studied in depth, as we summarize next. V. Fonf, V. Gurariy, and V. Kadeč in 1999 [144] showed that the set of nowhere differentiable functions on $[0,1]$ is spaceable. But much more is true: L. Rodríguez-Piazza showed that the $X$ in [144] can be chosen to be isometrically isomorphic to any separable Banach space [245]. Several authors have invested plenty of time on the study of this special set of functions since the ending of the twentieth century. For instance, S. Hencl [184. showed in 2000 that any separable Banach space is isometrically isomorphic to a subspace of $\mathcal{C}[0,1]$ whose nonzero elements are nowhere approximately differentiable (recall that if $I \subset \mathbb{R}$ is an interval and $x_{0} \in \mathbb{R}$, then a function $f: I \rightarrow \mathbb{R}$ is called approximately differentiable at $x_{0}$ provided that there is $\alpha \in \mathbb{R}$ such that, for each $\varepsilon>0$, the set $\left\{x \in E:\left|\frac{f(x)-f\left(x_{0}\right)}{x-x_{0}}-\alpha\right|<\varepsilon\right\}$ has $x_{0}$ as a density point) and nowhere Hölder. Bayart and Quarta produced the following result, which is related to the algebraic structure of this special set.

Theorem 2.1 (Bayart and Quarta, 2007 [54]). The set of continuous nowhere Hölder functions on $[0,1]$ contains (except for the null function) an infinitely generated algebra. Moreover, this algebra can be chosen to be dense in $\mathcal{C}[0,1]$. In other words, the set of continuous nowhere Hölder functions on $[0,1]$ is $\aleph_{0}$-densealgebrable.

From this last assertion one can infer that the set of continuous nowhere differentiable functions on $[0,1]$ is dense-lineable and, in particular, $\aleph_{0}$-lineable. Just recently, the authors of [191] provided the first constructive proof of the c-lineability of this set.

Finally, and in the vein of Theorem 1.3 by Gurariy, we mention that, if $\mathcal{C}^{\infty}(I)$ denotes the space of infinitely differentiable real functions on an interval $I \subset \mathbb{R}$, then obviously, $\mathcal{C}^{\infty}([0,1])$ is not spaceable in $\mathcal{C}[0,1]$. In spite of this fact, the class $\mathcal{C}^{\infty}((0,1))$ is spaceable in $\mathcal{C}((0,1))$. For this, see [68], where, in addition, the use of Müntz sequences allow us to prove that the family of continuous functions on $[0,1]$ which are analytic in $(0,1)$ is spaceable in $\mathcal{C}[0,1]$. Hence $\mathcal{C}[0,1] \cap \mathcal{C}^{\infty}((0,1))$ is spaceable as well.

2.2. Differentiable functions and related properties. Clearly, the set of everywhere differentiable functions on $\mathbb{R}$ is linear and, thus, $\mathfrak{c}$-lineable since it is itself a vector space. Gurariy showed in [178 that this cannot be improved: the set of everywhere differentiable functions on $[0,1]$ is not spaceable. In this section we shall discuss some special subsets of differentiable functions.

2.2.1. Differentiable nowhere monotone functions. The existence of differentiable functions on $\mathbb{R}$ that are nowhere monotone (denoted $\mathcal{D N} \mathcal{M}(\mathbb{R})$ from now on) is a 
well-known fact since the appearance of the example by Katznelson and Stromberg in 1974 196. Several more examples and constructions have followed since. One of the most recent constructions, if not the most recent, of such a function can be found in 22, where the authors make use of several technical lemmas in order to achieve two main goals. First, the construction of one such function and, second, the following result.

Theorem 2.2 (Aron, Gurariy, and Seoane-Sepúlveda, 2005 22]). The $\operatorname{set} \mathcal{D N M}(\mathbb{R})$ of differentiable functions on $\mathbb{R}$ which are nowhere monotone is $\aleph_{0}$-lineable in $\mathcal{C}(\mathbb{R})$.

The previous result was improved in 20, where the authors proved that the set of differentiable nowhere monotone function on any compact interval of $\mathbb{R}$ is actually dense-lineable, so showing that the vector space in 22 can be chosen to be dense.

Recall that $\operatorname{dim}(\mathcal{C}[0,1])$ and $\operatorname{dim}(\mathcal{C}(\mathbb{R}))$ are both equal to $\mathfrak{c}$. Thus we could also wonder whether the set $\mathcal{D N} \mathcal{M}(\mathbb{R})$ is $\mathfrak{c}$-lineable. The answer is yes, and it was obtained in [149, where the authors use approximately continuous functions and the properties of density topology 5 to obtain the above statement by means of the following statement. Recall that if $I \subset \mathbb{R}$ is an interval, then a function $f: I \rightarrow \mathbb{R}$ is said to be approximately continuous whenever, for every open set $U \subset \mathbb{R}$, the set $f^{-1}(U)$ is Lebesgue measurable and has Lebesgue density one at each of its points.

Theorem 2.3 (Gámez-Merino, Muñoz-Fernández, Sánchez, and Seoane-Sepúlveda, 2010 [149]). The set of bounded approximately continuous functions on $\mathbb{R}$ that are positive in a dense subset of $\mathbb{R}$ and negative in another dense subset of $\mathbb{R}$ is $\mathfrak{c}$ lineable.

Next, since every bounded approximately continuous mapping is the derivative of a differentiable function (see [117, Theorem 5.5(a), p. 21]), the authors obtain as an easy consequence of Theorem 2.3 the following assertion.

Theorem 2.4 (Gámez-Merino, Muñoz-Fernández, Sánchez, Seoane-Sepúlveda, $2010149)$. The set $\mathcal{D} \mathcal{N} \mathcal{M}(\mathbb{R})$ of differentiable functions on $\mathbb{R}$ that are nowhere monotone is c-lineable.

Due to the previously discussed result by Gurariy ([178] and Theorem 1.3 in Section 1), the set $\mathcal{D} \mathcal{N} \mathcal{M}[0,1]$ cannot be spaceable.

2.2.2. Nowhere analytic functions. As is standard, a real function is said to be real analytic if it possesses derivatives of all orders and agrees with its Taylor series in a neighborhood of every point. There exist $\mathcal{C}^{\infty}$ functions that are not analytic, as the following well-known function shows:

$$
f(x)=\left\{\begin{array}{cl}
e^{-1 / x^{2}} & \text { if } x \neq 0 \\
0 & \text { if } x=0
\end{array}\right.
$$

Since $f^{(n)}=0(n \geq 0)$, the above function only agrees with its Taylor series expansion at $x=0$. Hence $f$ belongs to $\mathcal{C}^{\infty}(\mathbb{R})$ but is not analytic at 0 . But more is true: in 1876 [95], du Bois-Reymond constructed a function belonging to $\mathcal{S}(\mathbb{R})$, the family of all everywhere singular functions, that is, the class of $C^{\infty}$-functions

\footnotetext{
${ }^{5}$ Density topology can be defined as the initial topology for approximately continuous functions.
} 
on $\mathbb{R}$ that are analytic at no point of $\mathbb{R}$. A nice example is the following one due to Lerch 210:

$$
f(x)=\sum_{n=1}^{\infty} \frac{\cos \left(a^{n} x\right)}{n !},
$$

where $a$ is an odd positive integer. Other explicit examples can be seen in [188, 200].

Again, this phenomenon is topologically generic: in 1955, Salzmann and Zeller 252 established the residuality of the family $\mathcal{S}(I)$ of everywhere singular functions on $I$ in the space $\mathcal{C}^{\infty}(I)$, endowed with the topology of uniform converge in compacta of functions and the derivatives of all orders, where $I \subset \mathbb{R}$ is an interval. We would also like to refer the reader to the nice work of Bastin, Esser, and Nikolay [42], where the authors study the genericity of functions which are nowhere analytic in a measure-theoretic sense.

Concerning lineability, García-Pacheco, Palmberg, and Seoane-Sepúlveda [156] demonstrated that there actually exists an uncountably infinitely generated algebra, every nonzero element of which is in $\mathcal{C}^{\infty}(\mathbb{R})$ and nonanalytic at $x=0$. Cater [121] showed in 1984 that, although the set of nowhere analytic functions on $[0,1]$ is clearly not a linear space, there exists a vector space in $\mathcal{S}([0,1]) \cup\{0\}$ of dimension c. Recently, Bernal 68 proved that there is a dense linear subspace in $\mathcal{C}^{\infty}[0,1]$, every nonzero element of which is nowhere analytic. Moreover, he showed 68, Theorem 3.2] that if $\mathcal{P} \mathcal{S}$ stands for the set of all smooth functions with a Pringsheim singularity at every point of $[0,1]$ (which is nonempty due to a classical result of Zahorski [274]), then $\mathcal{P S}$ is dense-lineable. Recall that a function $f \in \mathcal{C}^{\infty}(I)$ is said to have a Pringsheim singularity at a point $x_{0} \in I$ whenever the radius of convergence of the Taylor series of $f$ at $x_{0}$ is zero. Obviously, $\mathcal{P S} \subset \mathcal{S}([0,1])$, the inclusion being strict. In fact, Salzmann and Zeller had shown in 252 that $\mathcal{P} \mathcal{S}$ is residual. In [68 it is also proved that $\mathcal{S}([0,1])$ is maximal-dense-lineable and that if the arrival space is $\mathbb{C}$, then $\mathcal{P} \mathcal{S}$ is maximal-lineable.

If an entire function has infinitely many zeros with an accumulation point, then by the Identity Theorem it must be the zero function (see, e.g., [118, for a very accessible work on uniqueness theorems for analytic functions). For real differentiable functions this no longer holds. For instance, the differentiable function $g: \mathbb{R} \rightarrow \mathbb{R}$ given by

$$
g(x)=\left\{\begin{array}{cl}
x^{2} \sin (\pi / x) & \text { if } x \neq 0 \\
0 & \text { if } x=0
\end{array}\right.
$$

has the infinite set $Z=\left\{\frac{1}{n}: n \in \mathbb{N}\right\} \cup\{0\}$ as its set of zeros, $Z$ has an accumulation point (0) but, obviously, $g \neq 0$. This raises the following natural questions:

Are there real valued $C^{\infty}$-functions with infinitely many zeros, and which in addition are nowhere analytic? And, how big is this set of functions? What algebraic/linear structure does this set possess?

In [128, Theorem 2.3] the authors provide answers to the above questions by constructing an algebra $\mathcal{A}$ of real valued functions enjoying, simultaneously, each of the following properties:

(i) $\mathcal{A}$ is uncountably infinitely generated (that is, the cardinality of a minimal system of generators of $\mathcal{A}$ is uncountable).

(ii) Every nonzero element of $\mathcal{A}$ is nowhere analytic.

(iii) $\mathcal{A} \subset \mathcal{C}^{\infty}(\mathbb{R})$.

(iv) Every element of $\mathcal{A}$ has infinitely many zeros in $\mathbb{R}$. 
(v) For every $f \in \mathcal{A}$ and $n \in \mathbb{N}$, the $n$th derivative $f^{(n)}$ also possesses infinitely many zeros in $\mathbb{R}$.

Functions with infinitely many zeros in a closed finite interval are known as annulling functions; see 137, Definition 2.1]. Very recently, Enflo, Gurariy, and Seoane-Supúlveda [137, Corollary 3.8] proved that for every infinite dimensional subspace $X$ of $\mathcal{C}[0,1]$ the subset of its annulling functions contains an infinite dimensional closed subspace; see also Section 2.7.1. The question of the existence of an algebra of such functions inside of $\mathcal{C}[0,1]$ is also solved in [128. The following problems come naturally.

\section{Problems 2.5.}

1. Is the class $\mathcal{P} \mathcal{S}$-algebrable in $\mathcal{C}^{\infty}([0,1])$ ?

2. Can we replace the words "is nowhere analytic" in the property (ii) above by the condition "belongs to $\mathcal{P S}$ "?

Remark 2.6. Within the framework of vector-valued analytic functions, we refer the interested reader to the paper [213] by López-Salazar, where large vector spaces of entire functions of unbounded type are constructed.

\subsection{Continuous (and nowhere continuous) functions.}

2.3.1. Maxima and minima of functions in $\mathbb{R}$. In 240] it was proved that there exists a continuous function on $\mathbb{R}$ with a proper local maximum at each point of a dense subset of $\mathbb{R}$. One could ask whether the set of all functions enjoying this property, denoted $C M(\mathbb{R})$, is lineable. Apparently this is not true, the problem being that the proper local maxima become proper local minima for any negative multiple of $f$, with $f \in C M(\mathbb{R})$. If $f$ also had a dense set of proper local minima, then this problem would not arise. Let us denote by $C M m(\mathbb{R})$ the nonempty (see 135) set of continuous functions such that both of their sets of proper local minima and maxima are dense in $\mathbb{R}$.

Theorem 2.7 (García, Grecu, Maestre, and Seoane-Sepúlveda, 2010, 154]). There exists an infinite dimensional Banach space of continuous functions on $\mathbb{R}$ all of whose nonzero members have the property that their sets of proper local minima and maxima, respectively, are dense subsets of $\mathbb{R}$. In other words, CMm $(\mathbb{R})$ is spaceable. Moreover, $\lambda(C M m(\mathbb{R}))=\mathfrak{c}$ and $C M m(\mathbb{R})$ is $(\mathfrak{c}, \mathfrak{c})$-algebrable.

One of the first results in the topic of lineability was due to Gurariy and Quarta 181. They considered subsets of continuous functions attaining their maximum at exactly one point. To our surprise, sometimes one cannot achieve lineability for certain nontrivial sets, as the following result shows.

Theorem 2.8 (Gurariy and Quarta, 2004 [181]). Let $\mathcal{M}=\{f \in \mathcal{C}[0,1]: f$ attains its maximum at exactly one point of $[0,1]\}$.

(1) $\mathcal{M}$ is a dense $G_{\delta}$ set in $\mathcal{C}[0,1]$. In particular, $M$ is residual.

(2) If $V \subset \mathcal{M} \cup\{0\}$ is a vector space, then $\operatorname{dim}(V) \leq 1$.

In [181, the authors provided a number of partial extensions of Theorem 2.8. For instance, for both $\mathcal{C}(\mathbb{R})$ and $\mathcal{C}_{0}(\mathbb{R})$ (continuous functions $\mathbb{R} \rightarrow \mathbb{R}$ vanishing at $\pm \infty$ ), there is a 2-dimensional subspace, every nonzero element of which attains its maximum at exactly one point of $\mathbb{R}$. In the case of $\mathcal{C}_{0}(\mathbb{R})$, there is no 3-dimensional 
subspace having this property (181]). After all the effort invested in this class of functions, the following problem still remains open (see also [13]).

Problem 2.9. Is there an $n$-dimensional vector subspace of $\mathcal{C}(\mathbb{R})$, with $n>2$, every nonzero element of which attains its maximum at exactly one point of $\mathbb{R}$ ?

2.3.2. Sierpiński-Zygmund functions. As a consequence of the classic Luzin's Theorem, we have that for every measurable function $f: \mathbb{R} \rightarrow \mathbb{R}$, there is a measurable set $S \subset \mathbb{R}$, of infinite measure, such that $\left.f\right|_{S}$ is continuous. A natural question would be whether similar results could be obtained for arbitrary functions (not necessarily measurable). In other words, given any arbitrary function $f: \mathbb{R} \rightarrow \mathbb{R}$, can we find a "large" subset $S \subset \mathbb{R}$ for which $\left.f\right|_{S}$ is continuous? In 1922, Blumberg 94] provided an affirmative answer to this question.

Theorem 2.10 (Blumberg, 1922 94]). Let $f: \mathbb{R} \rightarrow \mathbb{R}$ be an arbitrary function. There exists a dense subset $S \subset \mathbb{R}$ such that the function $\left.f\right|_{S}$ is continuous.

Blumberg's proof of his theorem (see, e.g., [198, p. 154]) shows that the set $S$ above is countable. We could wonder whether we can choose the subset $S$ in Blumberg's theorem to be uncountable. A (partial) negative answer was given in 261] by Sierpiński and Zygmund.

Theorem 2.11 (Sierpiński and Zygmund, 1923 [261]). There exists a function $f: \mathbb{R} \rightarrow \mathbb{R}$ such that, for any set $Z \subset \mathbb{R}$ of cardinality $\mathfrak{c}$, the restriction $\left.f\right|_{Z}$ is not a Borel map (and, in particular, not continuous).

From now on, we shall say that a function $f: \mathbb{R} \rightarrow \mathbb{R}$ is a Sierpinski-Zygmund function if it satisfies the condition in Sierpiński-Zygmund's Theorem, and we denote

$$
\mathcal{S Z}=\{f: \mathbb{R} \rightarrow \mathbb{R}: f \text { is a Sierpiński-Zygmund function }\} .
$$

Let us recall some known results about the class $\mathcal{S Z}$. It is known that if the Continuum Hypothesis $(\mathrm{CH})$ holds, then the restriction of a function in $\mathcal{S Z}$ to any uncountable set cannot be continuous (see, e.g., [198, pp. 165, 166]). Also, CH is necessary in this frame. Shinoda proved in 1973 [257] that if Martin's Axiom and the negation of $\mathrm{CH}$ hold, then, for every $f: \mathbb{R} \rightarrow \mathbb{R}$ there exists an uncountable set $Z \subset \mathbb{R}$ such that $\left.f\right|_{Z}$ is continuous. The functions in $\mathcal{S Z}$ are never measurable and, although it is possible to construct them being injective, they are nowhere monotone in a very strong way: their restriction to any set of cardinality $\mathfrak{c}$ is not monotone. In 1997, Balcerzak, Ciesielski, and Natkaniec showed in 32 that, assuming the set-theoretical condition $\operatorname{cov}(\mathcal{M})=\mathfrak{c}$ (which is true under Martin's Axiom or $\mathrm{CH}$ ), there exists a Darboux function that is in $\mathcal{S Z}$ as well. They prove also that there exists a model of the ZFC (Zermelo-Fraenkel Axiom of Choice) in which there are no such functions (see also [125, 126, 239]). Later, Gámez-Merino, Muñoz-Fernández, Sánchez, and Seoane-Sepúlveda (2010) proved in [149. Theorems 5.6 and 5.10] that the set $\mathcal{S Z}$ is $\mathfrak{c}^{+}$-lineable and also $\mathfrak{c}$-algebrable. As a consequence, assuming that $\mathfrak{c}^{+}=2^{\mathfrak{c}}$ (which follows, for instance, from the Generalized Continuum Hypothesis or $\mathrm{GCH}), \mathcal{S Z}$ would be $2^{\mathfrak{c}}$-lineable. Also, in 2010 [150, Corollary 2.11], Gámez-Merino, Muñoz, and Seoane-Sepúlveda proved that $\mathcal{S Z}$ is actually $d_{\mathfrak{c}}$-lineable, where $d_{\mathfrak{c}}$ is a cardinal invariant defined as

$$
d_{\mathfrak{c}}=\min \left\{\operatorname{card} F: F \subset \mathbb{R}^{\mathbb{R}},\left(\forall \varphi \in \mathbb{R}^{\mathbb{R}}\right)(\exists f \in F)(\operatorname{card}(f \cap \varphi)=\mathfrak{c})\right\} .
$$


This cardinal can take as value any regular cardinal between $\mathfrak{c}^{+}$and $2^{\mathfrak{c}}$, depending of the set-theoretical axioms assumed. Later, in [40, Theorem 2.6], Bartoszewicz, Głąb, Pellegrino, and Seoane-Sepúlveda showed that $\mathcal{S Z}$ is actually $\kappa$-strongly algebrable for some $\mathfrak{c}^{+} \leq \kappa \leq 2^{\mathfrak{c}}$ if there is in $\mathfrak{c}$ an almost disjoint family of cardinality $\kappa$ (see Definition 2.12 below). Assuming either Martin's Axiom, $\mathrm{CH}$, or $2^{<\mathfrak{c}}=\mathfrak{c}$, this $\kappa$ can be chosen to be $2^{\mathfrak{c}}$, so we would have that $\mathcal{S Z}$ is $2^{\mathfrak{c}}$-strongly algebrable.

Definition 2.12. Let $S$ a be set of cardinality $\kappa$. We say that a family $\mathcal{F} \subset \mathcal{P}(S)$ is an almost disjoint family in $S$ if the following conditions hold:

(1) If $A \in \mathcal{F}$, then card $A=\kappa$.

(2) If $A, B \in \mathcal{F}, A \neq B$, then $\operatorname{card}(A \cap B)<\kappa$.

Until very recently, it was not known whether any additional set-theoretical assumptions were needed or not in order to show the $2^{\mathfrak{c}}$-strong algebrability (and the $2^{\mathfrak{c}}$-lineability) of $\mathcal{S Z}$. Nevertheless, in [152] the authors showed the following:

Theorem 2.13 (Gámez-Merino and Seoane-Sepúlveda 2012 [152]). Let $\kappa$ be a cardinal number such that $\mathfrak{c}^{+} \leq \kappa \leq 2^{\mathfrak{c}}$. The following are equivalent:

(a) $\mathcal{S Z}$ is $\kappa$-strongly algebrable.

(b) $\mathcal{S Z}$ is $\kappa$-algebrable.

(c) $\mathcal{S Z}$ is $\kappa$-lineable.

(d) There exists an additive group $\mathcal{G} \subset \mathcal{S Z} \cup\{0\}$ of size (i.e., cardinality) $\kappa$.

(e) There exists in $\mathfrak{c}$ an almost disjoint family of cardinality $\kappa$.

Now, we review a series of results on almost disjoint families, all of which can be found in 205]. On the one hand, recall that under ZFC there is an almost disjoint family of cardinality $\mathfrak{c}=2^{\aleph_{0}}$ in $\aleph_{0}$. On the other hand, the existence of an almost disjoint family of cardinality $2^{\aleph_{1}}$ in $\aleph_{1}$ is undecidable. Also, and under the set-theoretical assumption $2^{<\mathfrak{c}}=\mathfrak{c}$, there exists an almost disjoint family of cardinality $2^{\mathfrak{c}}$ in $\mathfrak{c}$.

Let us point out that, from these previous results, we infer that it is consistent with $\mathrm{ZFC}$ that $\mathcal{S Z} \cup\{0\}$ contains a vector space of dimension $2^{\mathfrak{c}}$. By means of the forcing technique, in [152] the authors proved that the contrary is also consistent; that is:

Theorem 2.14 (Gámez-Merino and Seoane-Sepúlveda 2012, [152]). The $2^{\mathfrak{c}}$-lineability (maximal lineability) of the set of Sierpinski-Zygmund functions is undecidable.

This would be the first time in which one encounters a (highly nontrivial!) undecidable proposition in this theory of lineability and spaceability.

2.3.3. Surjections, Darboux functions, and related properties. The following concepts, although well known in real analysis, can be found in [145, 193.

Definition 2.15. Let $f \in \mathbb{R}^{\mathbb{R}}$. We say that:

(1) $f \in \operatorname{ES}(\mathbb{R})$ ( $f$ is everywhere surjective) if $f(I)=\mathbb{R}$ for every nontrivial interval $I$.

(2) $f \in \operatorname{SES}(\mathbb{R})$ ( $f$ is strongly everywhere surjective) if $f$ takes all values $\mathfrak{c}$ times on any interval.

(3) $f \in \operatorname{PES}(\mathbb{R})$ ( $f$ is perfectly everywhere surjective) if for every perfect set $P, f(P)=\mathbb{R}$. 
(4) $f \in \mathrm{AC}(\mathbb{R})(f$ is almost continuous, in the sense of J. Stallings [262]) if every open set containing the graph of $f$ contains also the graph of some continuous function.

(5) If $h: X \rightarrow \mathbb{R}$, where $X$ is a topological space, $h \in \operatorname{Conn}(X)$ ( $h$ is a connectivity function) if the graph of $\left.h\right|_{C}$ is connected for every connected set $C \subset X$. (If $h \in \mathbb{R}^{\mathbb{R}}$, it is equivalent to say that its graph is connected.)

(6) $f \in \operatorname{Ext}(\mathbb{R})\left(f\right.$ is extendable) if there is a connectivity function $g: \mathbb{R}^{2} \rightarrow$ $[0,1]$ such that $f(x)=g(x, 0)$ for every $x \in \mathbb{R}$.

(7) $f \in \operatorname{PR}(\mathbb{R})$ ( $f$ is a perfect road function) if for every $x \in \mathbb{R}$ there is a perfect set $P \subset R$ such that $x$ is a bilateral limit point of $P$ and $\left.f\right|_{P}$ is continuous at $x$.

(8) $f \in \mathrm{PC}(\mathbb{R})$ ( $f$ is peripherally continuous) if for every $x \in \mathbb{R}$ and pair of open sets $U, V \subset \mathbb{R}$ such that $x \in U$ and $f(x) \in V$ there is an open neighborhood $W$ of $x$ with $\bar{W} \subset U$ and $f(\partial W) \subset V$.

(9) $f \in \mathrm{J}(\mathbb{R})$ ( $f$ is a Jones function) if its graph intersects every closed subset of $\mathbb{R}^{2}$ with uncountable projection on the $x$-axis (see [192]).

(10) $f \in \mathcal{Q}(\mathbb{R})$ if $f$ is a $\mathbb{Q}$-linear function on $\mathbb{R}$.

(11) $f \in \operatorname{Gr}(\mathbb{R})(f$ is a dense-graph function) if its graph $\{(x, f(x)): x \in \mathbb{R}\}$ is dense in $\mathbb{R}^{2}$.

(12) $f \in \mathcal{D}(\mathbb{R})$ ( $f$ is a Darboux function) if it has the "intermediate value property"; that is, for any two values $a$ and $b$ in the domain of $f$, and any $y$ between $f(a)$ and $f(b)$, there is some $c$ between $a$ and $b$ with $f(c)=y$.

In order to make all the above definitions clearer to the reader, we can picture some of them in the following diagram, which links most of the above classes (the proofs of the implications below are either trivial, or can be found in [145, 149, 150, 193]). In what follows, $A \rightarrow B$ means that the class $A$ is a subset of $B 6$

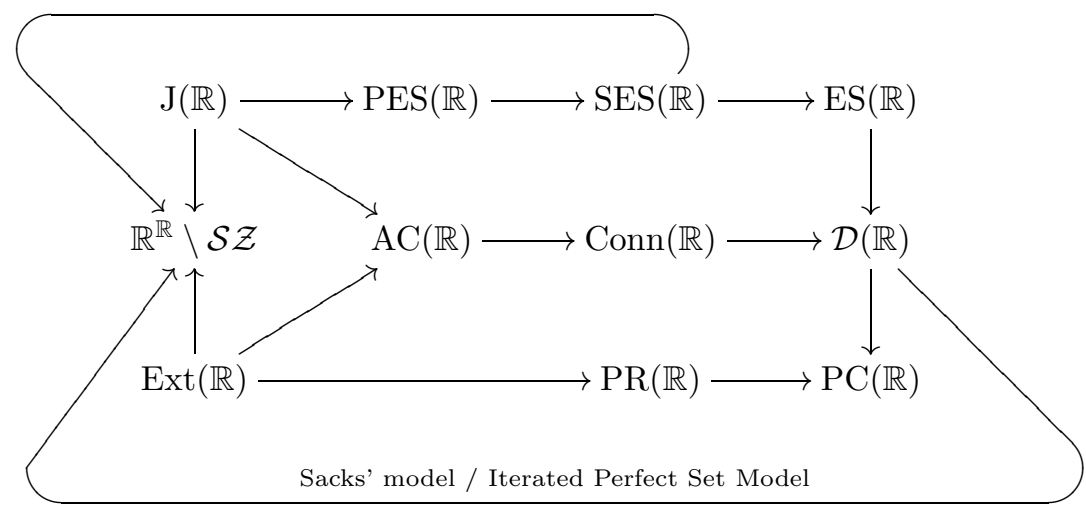

Lebesgue [161, 163, 206 was probably the first to show a somewhat surprising example of a function in $\operatorname{ES}(\mathbb{R})$. In 22 the authors proved that the set of such everywhere surjective functions is $2^{\mathfrak{c}}$-lineable, which is the best possible result in terms of dimension. One could think that, in terms of surjectivity, these everywhere

\footnotetext{
${ }^{6}$ Due to the highly technical set-theoretical background that it would require, we refer the interested reader to [127. for a complete and modern study of the so-called Iterated Perfect Set Model.
} 
TABLE 1

\begin{tabular}{|c|c|c|c|c|c|}
\hline CLASS & $\lambda$ & Ref. & CLASS & $\lambda$ & Ref. \\
\hline $\mathrm{AC}(\mathbb{R})$ & $2^{\mathfrak{c}}$ & 150 & $\mathrm{PC}(\mathbb{R})$ & $2^{\mathfrak{c}}$ & 22 \\
\hline $\operatorname{Conn}(\mathbb{R})$ & $2^{\mathfrak{c}}$ & 150 & $\mathcal{D N} \mathcal{M}(\mathbb{R})$ & $\mathfrak{c}$ & 22,149 \\
\hline $\operatorname{Ext}(\mathbb{R})$ & $\geq \mathfrak{c}^{+}$ & 150 & $\operatorname{PES}(\mathbb{R}) \backslash J(\mathbb{R})$ & $2^{\mathfrak{c}}$ & 149 \\
\hline $\mathrm{AC}(\mathbb{R}) \backslash \operatorname{Ext}(\mathbb{R})$ & $2^{\mathfrak{c}}$ & 150 & $\mathrm{SES}(\mathbb{R}) \backslash \mathrm{PES}(\mathbb{R})$ & $2^{\mathfrak{c}}$ & 149 \\
\hline $\operatorname{PR}(\mathbb{R})$ & $\geq \mathfrak{c}^{+}$ & 150 & $\operatorname{ES}(\mathbb{R}) \backslash \operatorname{SES}(\mathbb{R})$ & $2^{\mathfrak{c}}$ & 149 \\
\hline $\mathcal{S Z}$ & $d_{\mathfrak{c}}\left(" 2^{\mathfrak{c} "}\right)$ & 150,152 & $\mathcal{D}(\mathbb{R}) \backslash \mathrm{ES}(\mathbb{R})$ & $2^{\mathfrak{c}}$ & 149 \\
\hline $\mathrm{J}(\mathbb{R})$ & $2^{\mathfrak{c}}$ & 148 & $\mathrm{PC}(\mathbb{R}) \backslash \mathcal{D}(\mathbb{R})$ & $2^{\mathfrak{c}}$ & 149 \\
\hline $\operatorname{PES}(\mathbb{R})$ & $2^{\mathfrak{c}}$ & 149 & $\operatorname{ES}(\mathbb{R}) \cap \mathcal{Q}(\mathbb{R})$ & $2^{\mathfrak{c}}$ & 159 \\
\hline $\operatorname{SES}(\mathbb{R})$ & $2^{\mathfrak{c}}$ & 149 & $\operatorname{Gr}(\mathbb{R}) \cap \mathcal{Q}(\mathbb{R}) \backslash \mathrm{ES}(\mathbb{R})$ & $2^{\mathfrak{c}}$ & 159 \\
\hline $\mathrm{ES}(\mathbb{R})$ & $2^{\mathfrak{c}}$ & 22 & $\operatorname{ES}(\mathbb{R}) \backslash \mathcal{Q}(\mathbb{R})$ & $2^{\mathfrak{c}}$ & 159 \\
\hline $\mathcal{D}(\mathbb{R})$ & $2^{\mathfrak{c}}$ & 22 & $\operatorname{Gr}(\mathbb{R}) \backslash(\operatorname{ES}(\mathbb{R}) \cup \mathcal{Q}(\mathbb{R}))$ & $2^{\mathfrak{c}}$ & 159 \\
\hline
\end{tabular}

surjective functions are in some sense the most exotic. As the previous diagram shows, this is very far from being true.

For the sake of completeness and for the beauty of the construction, we provide here a proof of the existence of a function $f \in \operatorname{ES}(\mathbb{R})$.

Example 2.16 (An everywhere surjective function, [149, Example 2.2]). Let $\left(I_{n}\right)_{n \in \mathbb{N}}$ be the collection of all open intervals with rational endpoints. The interval $I_{1}$ contains a Cantor type set, call it $C_{1}$. Now, $I_{2} \backslash C_{1}$ also contains a Cantor type set, call it $C_{2}$. Next, $I_{3} \backslash\left(C_{1} \cup C_{2}\right)$ contains, as well, a Cantor type set, $C_{3}$. Inductively, we construct a family of pairwise disjoint Cantor type sets, $\left(C_{n}\right)_{n \in \mathbb{N}}$, such that for every $n \in \mathbb{N}, I_{n} \backslash\left(\bigcup_{k=1}^{n-1} C_{k}\right) \supset C_{n}$. Now, for every $n \in \mathbb{N}$, take any bijection $\phi_{n}: C_{n} \rightarrow \mathbb{R}$, and define $f: \mathbb{R} \rightarrow \mathbb{R}$ as

$$
f(x)= \begin{cases}\phi_{n}(x) & \text { if } x \in C_{n}, \\ 0 & \text { otherwise }\end{cases}
$$

Then $f$ is clearly everywhere surjective (and also zero almost everywhere!) Indeed, let $I$ be any interval in $\mathbb{R}$. There exists $k \in \mathbb{N}$ such that $I_{k} \subset I$. Thus $f(I) \supset$ $f\left(I_{k}\right) \supset f\left(C_{k}\right)=\phi_{k}\left(C_{k}\right)=\mathbb{R}$.

Let us now provide an account of the known lineability and algebrability results of some of the above classes (under ZFC). In Table 1, the letter $\lambda$ stands for the maximal (known) dimension of lineability of the given class.

The quotation marks above (" $2^{\mathfrak{c}}$ ", next to the class $\mathcal{S Z}$ ) refer to Theorem 2.14. Some of the results above follow from a result involving the concept of additivity [150, 193, which will be presented in detail in Part 5] of this survey.

Notice that some of the above classes are themselves subsets of the set of surjective functions from $\mathbb{R}$ to $\mathbb{R}$, and all of these subsets are $2^{\mathfrak{c}}$-lineable. One could think that a similar result would hold for one-to-one functions. In [253] a negative answer was given to this question.

Theorem 2.17 (Seoane-Sepúlveda, 2006 [149,253]). The set of injective functions is not lineable. Moreover, if $V$ is a vector space, every nonzero element of which is an injective function on $\mathbb{R}$, then $\operatorname{dim}(V)=1$.

The algebrability of the above classes (or modifications of them) have also been considered by many authors. As we mentioned already, to obtain algebrability is 
TABLE 2

\begin{tabular}{|c|c|c|}
\hline CLASS & ALGEBRABILITY DIMENSION & Ref. \\
\hline $\operatorname{ES}(\mathbb{C}), \operatorname{PES}(\mathbb{C})$ & $2^{\mathfrak{c}}$ & {$[18,27,[40]$} \\
\hline $\mathrm{SES}(\mathbb{C}) \backslash \operatorname{PES}(\mathbb{C})$ & $2^{\mathfrak{c}}$ & {$[37]$} \\
\hline $\mathcal{E D} \mathcal{D}(\mathbb{R})$ & $2^{\mathfrak{c}}$ & {$[37,[40]$} \\
\hline $\mathcal{E} \mathcal{D} \mathcal{F}(\mathbb{R})$ & $2^{\mathfrak{c}}$ & {$[37]$} \\
\hline $\mathcal{E D C}(\mathbb{R})$ & $2^{\mathfrak{c}}$ & {$[37]$} \\
\hline
\end{tabular}

highly more complex than to obtain lineability. For instance, it is out of place to consider algebras of functions in $\operatorname{ES}(\mathbb{R})$, since given any $f \in \mathbb{R}^{\mathbb{R}}$ we have that $f^{2} \notin \operatorname{ES}(\mathbb{R})$. The same happens for the classes $\operatorname{PES}(\mathbb{R}), \operatorname{SES}(\mathbb{R}), \mathrm{J}(\mathbb{R})$, and many others. But sometimes a dual of the previous classes can be certainly constructed in $\mathbb{C}^{\mathbb{C}}$. For instance, and just to cite very recent results on algebrability, let us consider Table 2 .

In Table 2 the classes $\mathcal{E D D}(\mathbb{R}), \mathcal{E D} \mathcal{F}(\mathbb{R})$, and $\mathcal{E D C}(\mathbb{R})$ denote, respectively, the set of everywhere discontinuous Darboux functions, the set of nowhere continuous functions having finitely many values, and the set of nowhere continuous functions mapping compact sets to compact sets. These last two classes were also thoroughly studied in 151] (see Theorem 2.19 from Section 2.3.4 in this survey).

Remark 2.18. Turning to surjectivity, but this time in the setting of families of functions defined on complex domains, a simple Baire-category argument shows that if $X$ is an infinite dimensional Banach space and $f: \mathbb{D} \rightarrow X$ is continuous $(\mathbb{D}:=\{z \in \mathbb{C}:|z|<1\}$, the open unit disc), then $f$ cannot be surjective. Let $H(\mathbb{D}, X):=\{f: \mathbb{D} \rightarrow X: f$ is holomorphic on $\mathbb{D}\}$. In 1976, Glovebnik [166] and independently Rudin [248] proved that the set $\mathcal{D}:=\{f \in H(\mathbb{D}, X): f(\mathbb{D})$ is dense in $X\}$ is not empty. Very recently, López-Salazar 214 has been able to demonstrate the lineability of $\mathcal{D}$.

2.3.4. Other properties related to the lack of continuity. It is standard that continuous functions transform compact or connected sets into compact or connected sets, respectively. It is interesting to ask whether this characterizes continuity or not. In other words, if a function $f: \mathbb{R} \rightarrow \mathbb{R}$ transforms compact sets into compact sets and connected sets into connected sets, can we expect $f$ to be continuous? This question was positively answered in the late 1960s by Hamlett [182] and White [271] (see also Velleman [268, Theorem 2]). Thus, the fact that $f$ satisfies only one of the following two conditions,

(1) $f$ transforms compact sets into compact sets, or

(2) $f$ transforms connected sets into connected sets,

might be very far away from making $f$ continuous, which is what the authors proved in [151]:

Theorem 2.19 (Gámez-Merino, Muñoz-Fernández and Seoane-Sepúlveda, 2011 [151]). There exist $2^{\mathfrak{c}}$-dimensional linear spaces $U$ and $V$ of $\mathbb{R}^{\mathbb{R}}$ such that:

(a) Every nonzero element of $U$ is nowhere continuous and transforms connected sets into connected sets.

(b) Every nonzero element of $V$ is nowhere continuous and transforms any set into a compact set. 
Next, one could also study noncontinuous functions focusing on the structure of their sets of discontinuities. To start with, the set of points at which a function is continuous is always a $G_{\delta}$ set, so the set of discontinuities is an $F_{\sigma}$ set. Moreover, the set of discontinuities of a monotonic function is at most countable (Froda's theorem). In this direction, several results have been obtained in recent years. Recall that a point $x_{0} \in \mathbb{R}$ is called a removable discontinuity of a function $f: \mathbb{R} \rightarrow$ $\mathbb{R}$ whenever there exists $\lim _{x \rightarrow x_{0}} f(x)$ and is finite.

Theorem 2.20 (García-Pacheco, Palmberg, and Seoane-Sepúlveda, 2007 [156]). The set $M$ of functions on $\mathbb{R}$ with a dense set of points of removable discontinuity is $(\mathfrak{c}, \mathfrak{c})$-algebrable and $\lambda(M) \geq \mathfrak{c}$.

In this same direction, the following battery of results is also related to the set of discontinuities of functions in $\mathbb{R}$ (see [2,156]).

Theorem 2.21. (1) Given a closed set $F \subset \mathbb{R}$, the set $H$ of all functions $\mathbb{R} \rightarrow \mathbb{R}$, whose set of points of discontinuity is $F$, is lineable with $\lambda(H) \geq \mathfrak{c}$. Moreover, if the interior $F^{\circ} \neq \varnothing$, then $\lambda(H)=2^{\mathfrak{c}}$.

(2) Given any nonclosed $F_{\sigma}$ set $F$, the set of functions whose set of points of discontinuity is $F$, is coneable.

(3) Given a closed set $F$ of measure zero contained in an interval $[a, b]$, the set of all Riemann integrable functions whose set of points of discontinuity is $F$, is lineable.

(4) Given any nonclosed $F_{\sigma}$ set $F$ of measure zero contained in an interval $[a, b]$, the set of all Riemann-integrable functions whose set of points of discontinuity is exactly $F$, is coneable.

(5) Let $I$ be any nontrivial interval, and consider a point $a \in I$. Let $K$ denote the set of all functions from $I$ to $\mathbb{R}$ having a removable discontinuity at a. Then, $\lambda(K)=1$. If $L$ denotes the set of all functions from $I$ to $\mathbb{R}$ having a jump discontinuity at a, then $\lambda(L)=1$. Also, if $H$ denotes the set of all functions from $I$ to $\mathbb{R}$ having either a removable or jump discontinuity at $a$, then $\lambda(H)=2$.

\subsection{Measurability and integration.}

2.4.1. Nonmeasurable functions. The existence of nonmeasurable functions is a direct consequence of the famous Vitali's set $V$, for whose construction the Axiom of Choice is used. Recall that $V$ is defined by choosing one point in each class corresponding to the equivalence relation in $[0,1]$ given by $x \sim y$ if and only if $x-y \in \mathbb{Q}$. Then $\chi_{V}$ is "our" example of a nonmeasurable function. Let us now move to the search for Banach spaces of nonmeasurable functions. If $\mathbb{K}$ stands for either $\mathbb{R}$ or $\mathbb{C}$, then for a given a measurable space $(\Omega, \Sigma), N(\Omega, \mathbb{K})$ will denote the set of nonmeasurable functions from $\Omega$ to $\mathbb{K}$. By the Axiom of Choice, we know that $N(\Omega, \mathbb{K})$ is not empty.

In 2006, the authors of [160] obtained the best possible result - in terms of dimension and topological structure - when they provided maximal spaceability of the above set. In particular, we have the following theorem. Recall that if $X$ is a topological space and $\gamma$ is a cardinal, then $X$ is said to have density character $\gamma$ if $\gamma$ is the minimum cardinality of a dense subset of $X$.

Theorem 2.22 (García-Pacheco and Seoane-Sepúlveda, 2006 [160]). For any cardinal $\gamma$ there is a Hausdorff topological space $\Omega$ with Borel $\sigma$-algebra $\mathcal{B}$ such that 
$N(\Omega, \mathbb{K}) \cup\{0\}$ contains a subspace isometric to $\ell_{\infty}(\gamma)$. In particular, $N(\Omega, \mathbb{K})$ is spaceable, and any Banach space with density character $\gamma$ is isometric to a space consisting, except for zero, of nonmeasurable functions.

2.4.2. $L_{p}$ and $\ell_{p}$ spaces. The search of algebraic structures inside certain subsets of spaces of either $L_{p}$ or $\ell_{p}$ probably started when H. Rosenthal [247] showed in 1978 that $c_{0}$ is quasi-complemented in $\ell_{\infty}$ (a closed subspace $Y$ of a Banach space $X$ is said to be quasi-complemented if there is a closed subspace $Z$ of $X$ such that $Y \cap Z=\{0\}$ and $Y+Z$ is dense in $X)$. This clearly implies that $\ell_{\infty} \backslash c_{0}$ is spaceable. Later, García-Pacheco, Martín and Seoane-Sepúlveda proved (2009 [155]) that $\ell_{\infty}(\Gamma) \backslash c_{0}(\Gamma)$ is spaceable for every infinite set $\Gamma$, although it is interesting to recall that J. Lindenstrauss (1968 [212]) proved that if $\Gamma$ is uncountable, then $c_{0}(\Gamma)$ is not quasi-complemented in $\ell_{\infty}(\Gamma)$.

In 2008, Muñoz-Fernández, Palmberg, Puglisi, and Seoane-Sepúlveda [231] proved that if $I$ is a bounded interval and $q>p \geq 1$, then $L_{p}(I) \backslash L_{q}(I)$ is $\mathfrak{c}$ lineable. In this same paper it is proved that both, $\ell_{p} \backslash \ell_{q}$ and $L_{p}(J) \backslash L_{q}(J)$, are $\mathfrak{c}$-lineable for any unbounded interval $J$ and for $p>q \geq 1$. One year later, Aron, García-Pacheco, Pérez-García, and Seoane-Sepúlveda [20] showed that the linear subspaces constructed in [231] can be chosen to be dense. In 2010, Bernal [69] provided a series of conditions from which one can obtain (maximal) lineability (and dense-lineability) of the set of functions in $L_{p}(X, \mu)$ that are not in $L_{q}(X, \mu)$, where $1 \leq q \neq p<\infty$ and $\mu$ denotes a regular Borel measure on a topological space $X$. And García-Pacheco, Pérez-Eslava, and Seoane-Sepúlveda [157, Theorem 2.6] proved that if $(\Omega, \Sigma, \mu)$ is a measure space such that there exists $\varepsilon>0$ and an infinite family $\left(A_{n}\right)_{n \in \mathbb{N}} \subset \Sigma$ of pairwise disjoint measurable sets with $\mu\left(A_{n}\right) \geq \varepsilon$ for all $n \in \mathbb{N}$, then $\bigcap_{p=1}^{\infty}\left(L_{\infty}(\Omega) \backslash L_{p}(\Omega)\right)$ is spaceable in $L_{\infty}(\Omega)$.

These previous results kept evolving and, in 2011, Botelho, Diniz, Fávaro, and Pellegrino [109 proved that if $X$ is a fixed Banach space, then for large classes of Banach (and even quasi-Banach) spaces $E$ of $X$-valued sequences, the sets $E \backslash \bigcup_{q \in \Gamma} \ell_{q}(X)$ (where $\Gamma \subset[0, \infty)$ ) and $E \backslash c_{0}(X)$ are both spaceable in $E$. Next, and as a consequence of a lecture delivered by V. Fávaro at an international conference held in Valencia, Spain, in 2010, R. Aron asked whether the result above [109, Corollary 1.7] would hold for $L_{p}$-spaces. This question was answered in the positive (and independently) in [84,111]. More precisely, in 84. Bernal-González and Ordóñez-Cabrera provided a series of conditions on a measure space $(\Omega, \Sigma, \mu)$ to ensure the spaceability of the sets $L_{p}(\Omega) \backslash \bigcup_{q \in[1, p)} L_{q}(\Omega), L_{p}(\Omega) \backslash \bigcup_{q \in[p, \infty)} L_{q}(\Omega)$, and $L_{p}(\Omega) \backslash \bigcup_{q \in[1, \infty) \backslash\{p\}} L_{q}(\mu, X)$ (for $p \geq 1$ ); whereas in [111] Botelho, Fávaro, Pellegrino, and Seoane-Sepúlveda obtained a quasi-Banach version of this result by proving that $L_{p}[0,1] \backslash \bigcup_{q>p} L_{q}[0,1]$ is spaceable for every $p>0$. In this direction it is also crucial to mention a recent paper 201, where Kitson and Timoney provided a general result from which some of the above results (for the normed case) can be inferred. On the opposite side, a celebrated old theorem due to Grothendieck (see [250, Chap. 6]) asserts that if $0<p<+\infty$ and $\mu$ is finite, then $L_{\infty}(\Omega)$ is not spaceable in $L_{p}(\Omega)$.

At this point, and after all the invested effort for the past years in looking for the "optimal" results on the spaceability of the sets of the form $L_{p}(\Omega) \backslash L_{q}(\Omega)$ with $p>q$ and $L_{p}(\Omega) \backslash \bigcup_{1 \leq q<p} L_{q}(\Omega)$, this ongoing work continued, and rather conclusive contributions in the form of maximal-spaceability have been obtained. 
Let again $(\Omega, \Sigma, \mu)$ denote a measure space and $0<p<+\infty$. Let us recall the following definitions, some of which can be found in 108 .

Definition 2.23. $\quad$ (i) $\Sigma_{\mathrm{fin}}:=\{A \in \Sigma: \mu(A)<+\infty\}$.

(ii) Two sets $A, B \in \Sigma_{\text {fin }}$ are equivalent, denoted $A \sim B$, if $\mu((A \backslash B) \cup(B \backslash A))=$ 0.

The elements of the quotient set $\Sigma_{\mathrm{fin}} / \sim$ are denoted by $[B]$, for $B \in \Sigma_{\mathrm{fin}}$.

(iii) The cardinal number $\#^{\Sigma_{\mathrm{fin}}} / \sim$ is called the entropy of the measure space $(\Omega, \Sigma, \mu)$ and is denoted by $\operatorname{ent}(\Omega)$.

(iv) Given a cardinal number $\zeta$, the measure space $(\Omega, \Sigma, \mu)$ is said to be $\zeta$ bounded if, for every $A \in \Sigma_{\text {fin }}$ with positive measure, there are at most $\zeta$ subsets of $A$ with positive measure belonging to different classes of $\Sigma_{\text {fin }} / \sim$.

Theorem 2.24 (Botelho, Cariello, Fávaro, Pellegrino, and Seoane-Sepúlveda, 2012 $\left[108\right.$ ). Let $p>1$. The set $L_{p}(\Omega) \backslash \bigcup_{1 \leq q<p} L_{q}(\Omega)$ is maximal-spaceable if at least one of the following conditions holds:

(a) either $L_{p}(\Omega) \backslash L_{r}(\Omega) \neq \varnothing$ for some $1 \leq r<p$ and $\aleph_{0} \leq \operatorname{ent}(\Omega) \leq \mathfrak{c}$,

(b) or the measure space $(\Omega, \Sigma, \mu)$ is $\zeta$-bounded for some cardinal number $\zeta$ with $\mathfrak{c} \leq \zeta<\operatorname{ent}(\Omega)$.

However, the authors also showed that $L_{p}(\Omega) \backslash L_{q}(\Omega)$ may fail to be maximalspaceable for $p>q$; see [108, Theorem 4.4]. They were able to show that that there exist (quite exotic) infinite measure spaces $(\Omega, \Sigma, \mu)$ such that $L_{p}(\Omega) \backslash L_{q}(\Omega)$, $q<p$, fails to be maximal-spaceable. They did this by developing a hybridization technique allowing them to prove much more: given $1 \leq q<p$ and cardinal numbers $\kappa>\zeta \geq \mathfrak{c}$, the authors constructed an infinite measure space $(\Omega, \Sigma, \mu)$ such that:

(i) $\operatorname{dim}\left(L_{p}(\Omega)\right)=\kappa$;

(ii) $\zeta$ is the maximal dimension of a closed subspace of $L_{p}(\Omega)$ contained (except for the null vector) in $L_{p}(\Omega) \backslash L_{q}(\Omega)$.

Before finishing this section, let us point out that (very recently) some results related to the problems treated in this section have been obtained by Barroso, Botelho, Fávaro, and Pellegrino in [36, and by Głąb, Kaufmann, and Pellegrini 164, 165. Within the framework of Orlicz spaces, we also refer the reader to the very recent work by Akbarbaglu and Maghsoudi [4.

2.4.3. Riemann versus Lebesgue. Given an interval $I$ (bounded or not), we shall denote by $\mathcal{R}(I)$ to the set of Riemann-integrable functions on $I$ and by $\mathcal{L}(I)$ the set of Lebesgue-integrable functions on $I$. The well known theorem by Lebesgue about Riemann-integrability states that if $I$ is a bounded interval and $f: I \longrightarrow \mathbb{R}$ is a bounded function, then $f$ is Riemann-integrable if and only if $f$ is almost everywhere continuous. The proof can be easily adapted to show that a Riemannintegrable function on any arbitrary interval (bounded or not) is always almost everywhere continuous.

One of the key points that led Lebesgue to his theory of integration was the existence of two examples, one given by Volterra in 1881 and another by Brodén in 1896 which showed that, at least from the point of view of Riemann integration, the process of obtaining antiderivatives of a function and integration theory were not equivalent. In 1881 Volterra gave an example of a differentiable function on $\mathbb{R}$ whose derivative is bounded but not Riemann-integrable (see [154] for a detailed 
construction of this function). Also, in 1896, Brodén gave an example of a real valued function $f$ being continuous, nonconstant, differentiable, and with $f^{\prime}$ bounded and zero in a dense subset of $\mathbb{R}$.

Theorem 2.25 (García, Grecu, Maestre, and Seoane-Sepúlveda, 2010 [154]). There exists an infinite dimensional Banach algebra of (except for 0) Brodén-type functions. In particular, the set of Brodén-type functions is spaceable and algebrable.

Notice that the derivative of any Brodén-type function is bounded but not Riemann-integrable. We say that a bounded function $f$ has property $(\star)$ if there exists a function $F$ such that $F^{\prime}(x)=f(x)$ for all $x \in \mathbb{R}$ but $f$ is not Riemannintegrable on any compact interval of $\mathbb{R}$. In particular, any function enjoying $(\star)$ does not satisfy the Fundamental Theorem of Calculus. In [154] the authors showed the following.

Theorem 2.26 (García, Grecu, Maestre, and Seoane-Sepúlveda, 2010 [154]). Given an interval $[a, b], a<b$, there exists an infinite dimensional Banach space of bounded functions which are Lebesgue-integrable, have antiderivatives at every point of $[a, b]$ but (except for 0 ) are not Riemann-integrable on $[a, b]$.

Following this direction of results on Lebesgue and Riemann integration, the following results were also recently obtained [155].

Theorem 2.27 (García-Pacheco, Martín, and Seoane-Sepúlveda, 2009 [155]). Let $I$ be any arbitrary unbounded interval. Then:

(a) The set of all almost everywhere continuous bounded functions on I which are not Riemann-integrable contains an infinitely generated closed subalgebra (in particular this set is spaceable and algebrable) in the Banach space $\mathcal{B}(I)$ of bounded functions $I \longrightarrow \mathbb{R}$, endowed with the supremum norm.

(b) The set of all continuous bounded functions on I which are not Riemannintegrable is spaceable in $\mathcal{B}(I)$.

Recall that if $I$ is an unbounded interval, then $\mathcal{R}(I) \nsubseteq \mathcal{L}(I)$; a representative and classical example is given by the function $f(x)=\frac{\sin x}{x}$ for every $x \in \mathbb{R}$. This function satisfies that

$$
\int_{\mathbb{R}} f(x) d x=\pi \quad \text { and } \quad \int_{\mathbb{R}}|f(x)| d x=+\infty .
$$

Conversely, on any interval $I$ (bounded or unbounded), there is a bounded Lebesgue-integrable function which is not equivalent (in the sense of the Lebesgue measure) to any Riemann-integrable function. An easy example of this type can be found in [163, Example 8.31]. Indeed, in any interval $I$, take a Cantor set $A \subset I$ with positive and finite measure (see, e.g., [163, Example 8.4]); then, the function $f=\chi_{A}$ is bounded, Lebesgue-integrable, but it is not equivalent to any Riemannintegrable function, since we can observe that $f=0$ in $I \backslash A$, which is dense in $I$ and, moreover, if we change $f$ in a null-set $B$ of $I$, then $f=0$ in the still dense subset $I \backslash(A \cup B)$, and $f=1$ in the set of positive measure $A \backslash B$.

Within the framework of Lebesgue integration in $\mathbb{R}$, we have the following.

Theorem 2.28 (García-Pacheco, Martín, and Seoane-Sepúlveda, 2009 [155]). Given any unbounded interval $I$, the set of Riemann-integrable functions on $I$ that are not Lebesgue-integrable is lineable. Also, given any interval I, the set of Lebesgueintegrable functions that are not Riemann-integrable is spaceable. 
Observe that the second part of the previous theorem - that is, the spaceability of $\mathcal{L}(I) \backslash \mathcal{R}(I)$ - is a consequence of a stronger result ([154, §4], Theorem 2.26 above). Also, notice that it is not possible to obtain any kind of algebrability of $\mathcal{R}(I) \backslash \mathcal{L}(I)$. Indeed, for every $f \in \mathcal{R}(I)$, either $f^{2} \notin \mathcal{R}(I)$ or $f^{2}=\left|f^{2}\right| \in \mathcal{R}(I)$ and, therefore, $f^{2} \in \mathcal{L}(I)$.

2.4.4. The composite function. Most of the examples existing (and presented in this survey) provide positive cases of high dimension in lineability but, sometimes, nice properties (such as continuity or Riemann-integrability) can be lost in a linear fashion via composition of functions. It is well known that, if $f$ is a continuous function on the interval $[a, b], g$ is Riemann-integrable (resp. Lebesgue-measurable) on the interval $[\alpha, \beta]$, and $g([\alpha, \beta]) \subset[a, b]$, then $f \circ g$ is Riemann-integrable (resp. measurable) on $[\alpha, \beta]$. A well-known fact, on the other hand, states that $f \circ g$ might be not Riemann-integrable (resp. measurable) when $f$ is Riemann-integrable (resp. measurable) and $g$ is continuous.

In [29] the authors proved that there exists a $2^{\mathfrak{c}}$-dimensional space $V$ and a $\mathfrak{c}$ dimensional space $W$ of, respectively, Riemann-integrable functions and continuous functions such that, for every $f \in V \backslash\{0\}$ and $g \in W \backslash\{0\}, f \circ g$ is not Riemannintegrable. They also proved that there exists a c-dimensional space $W$ of continuous functions such that for each $g \in W \backslash\{0\}$ there exists a $\mathfrak{c}$-dimensional space $V$ of measurable functions such that $f \circ g$ is not measurable for all $f \in V \backslash\{0\}$. It would be interesting to prove an analogue of their first result for measurable functions, that is:

Problem 2.29. Can one construct vector spaces (or even algebras!) $V$ and $W$ of measurable and continuous functions, respectively, such that $\operatorname{dim}(V)=2^{\mathfrak{c}}$, $\operatorname{dim}(W)=\mathfrak{c}$ and $f \circ g$ is nonmeasurable for every $f \in V \backslash\{0\}$ and every $g \in W \backslash\{0\} ?$

\subsection{Series and summability.}

2.5.1. Fourier series. The convergence of Fourier series has been deeply studied in the past. It came as a considerable surprise when du Bois-Reymond produced an example of a continuous function $f: \mathbb{T} \rightarrow \mathbb{C}$ whose Fourier series is divergent at one point of the unit circle $\mathbb{T}:=\left\{e^{i \theta}: \theta \in[0,2 \pi]\right\}$ (see [203, pp. 67-73] for a modern reference). This statement can be improved by means of an example of a continuous function whose Fourier series expansion diverges on a set of measure zero ([195, p. 58]). This last result is the best possible since, by a remarkable result due to Carleson (see, e.g., [203, p. 75]), the Fourier expansion of every continuous function converges almost everywhere. Moreover, by means of Baire's theorem, this exotic behavior can be shown to be generic: there exists a $G_{\delta}$ dense subset $E \subset \mathbb{T}$ such that the set of continuous functions whose Fourier expansion diverges on this set is a $G_{\delta}$ dense subset of $\mathcal{C}(\mathbb{T})$; see [249, p. 102]. F. Bayart 44] showed in 2005 that, if $\mathcal{F}_{E} \subset \mathcal{C}(\mathbb{T})$ is the set of continuous functions whose Fourier series expansion diverges on a prescribed set $E$ of measure zero, then $\mathcal{F}_{E}$ is dense-lineable. Later, in [25], the authors showed that this set $\mathcal{F}_{E}$ is actually dense-algebrable. There are functions in $L_{1}(\mathbb{T})$ with nowhere convergent Fourier series. In 2005, Bayart [43, Theorem 3] proved that $\mathcal{F}_{E}$ is, in addition, spaceable. Lineability properties in $\mathcal{C}(\mathbb{T})$ for Fourier series exhibiting universality phenomena have been recently shown; see 70 and Section 3.2. 
2.5.2. Dirichlet series. Another classical family of series is that of Dirichlet series, $\mathcal{H}^{\infty}$. These series are defined as

$$
f(s)=\sum_{n \in \mathbb{N}} a_{n} n^{-s},
$$

with convergence and boundedness of $f$ in the half-plane $\mathbb{C}_{+}=\{s \in \mathbb{C}: \Re(s)>0\}$; $\mathcal{H}^{\infty}$ is a Banach space with the sup norm over $\mathbb{C}_{+}$. It is known (see [50]) that there exists Dirichlet series $f(s)$ such that

$$
\sum_{n \in \mathbb{N}} a_{n} n^{-i t}
$$

diverges for every $t \in \mathbb{R}$. In 43 it was proved that the set of these Dirichlet series $f(s)$ such that $f(i t)$ diverges for every $t \in \mathbb{R}$ is spaceable (for further studies on this type of series we refer the interested reader to [116, 138]).

2.5.3. General summability. Sequence spaces. For some of the results in summability in sequence spaces of the $\ell_{p}$-type, we refer to Section 2.4.2 Let us now review some recent results surrounding subsets of scalar series. Let $\mathbb{K}=\mathbb{R}$ or $\mathbb{C}$. Also, let $C C(\mathbb{K})$ denote the set of conditionally convergent series (clearly, $C C(\mathbb{K}) \cup\{0\}$ is not a vector space in $C S(\mathbb{K})$, the set of convergent series). Aizpuru, PérezEslava, Seoane-Sepúlveda $(2006[3])$ showed that $C S(\mathbb{K})$ contains a vector space $E$ satisfying the following properties:

(i) every $x \in E \backslash\{0\}$ is a conditionally convergent series,

(ii) $\operatorname{dim}(E)=\mathfrak{c}$, and

(iii) $\operatorname{span}\left\{E \cup c_{00}\right\}$ is an algebra, and its elements are either elements of $c_{00}$ or conditionally convergent series.

Here, the symbol $c_{00}$ denotes the set of sequences, each of which has only finitely many nonzero terms. In the same work, the authors also showed that there exists a vector space $E \subset B S(\mathbb{K})$ (the set of all series with bounded partial sums) such that

(a) every $x \in E \backslash\{0\}$ is a divergent series,

(b) $\operatorname{dim}(E)=\mathfrak{c}$ and $E$ is nonseparable, and

(c) $\operatorname{span}\left\{E \cup c_{00}\right\}$ is an algebra and every element of it is either a divergent series or is an element of $c_{00}$.

In the same line of results, it is also proved in 3 that there exists a vector space $E \subset \ell_{\infty}$ such that

(1) $\operatorname{dim}(E)=\mathfrak{c}$,

(2) every $x \in E \backslash\{0\}$ is a divergent sequence,

(3) $E \oplus c_{0}$ is an algebra,

(4) every element in $\bar{E}+c_{0}$ is either a divergent sequence or a $c_{0}$-sequence, where $\bar{E}$ is the closure of $E$ in $\ell_{\infty}$.

Recall that if $X$ is a Banach space and $\sum_{i} x_{i}$ is a series in $X$, then it is said that $\sum_{i} x_{i}$ is unconditionally convergent (UC) provided that, for every permutation $\pi$ of $\mathbb{N}$, the series $\sum_{i=1}^{\infty} x_{\pi(i)}$ converges. And we say that $\sum_{i} x_{i}$ is weakly unconditionally Cauchy (WUC) if $\sum_{i=1}^{\infty}\left|f\left(x_{i}\right)\right|<\infty$ for every $f \in X^{*}$, the dual space of $X$. It is known [91,133] that if $X$ is a Banach space, then there exists a WUC series in $X$ which is convergent but is unconditionally convergent if and only if $X$ contains a copy of $c_{0}$. It is a also a well-known fact that every infinite dimensional Banach 
space has a series $\sum_{i} x_{i}$ which is unconditionally convergent and so that $\sum_{i}\left\|x_{i}\right\|=$ $\infty$; see [136. By $l_{1}^{\omega}\left(c_{0}\right)$ we will denote the space of all weakly unconditionally Cauchy series in $c_{0}$.

Theorem 2.30 (Aizpuru, Pérez-Eslava, Seoane-Sepúlveda, 2006 [3]). There exists a vector space $E \subset l_{1}^{\omega}\left(c_{0}\right)$ enjoying the following properties:

(a) $\operatorname{dim}(E)=\mathfrak{c}$, and

(b) if $x \in E \backslash\{0\}$, then $\sum_{i} x_{i}$ is not weakly convergent.

The authors also showed in 3 that, given any Banach space $X$, there exists a vector subspace $E$ of the space $U C(X)$ of unconditionally convergent series in $X$ such that $\operatorname{dim}(E)=c$, and if $x \in E \backslash\{0\}$, then $\sum_{i}\left\|x_{i}\right\|=\infty$. Notice that, from Theorem 2.30, it follows that $X$ has a copy of $c_{0}$ if and only if there exists a vector subspace $E$ of $\ell_{1}^{\omega}(X)$ with $\operatorname{dim}(E)=\mathfrak{c}$, so that every nonzero element of $E$ is a nonweakly convergent series.

Recently, in [38] the authors showed that the set of conditionally convergent real series considered with the Cauchy product is actually $\left(\aleph_{0}, 1\right)$-algebrable by means of a classical result due to Pringsheim (1883 241]). We also refer the interested reader to the recent work by Bartoszewicz, Głąb, and Poreda 41, in which they study the algebrability of some of the above mentioned classes.

2.6. Nonextendable holomorphic functions. In 1884 Mittag-Leffler proved that each domain in $\mathbb{C}$ supports a holomorphic function that is not holomorphically continuable to any larger domain. This surprising phenomenon can be studied from the generic point of view, in both topological and algebraic aspects. We start with the precise definitions.

2.6.1. Definitions and topological genericity. Let $N \in \mathbb{N}$ and consider the space $\mathbb{C}^{N}=\mathbb{C} \times \cdots \times \mathbb{C}(N$-fold $)$, which is a metric space under the distance $d(z, w)=$ $\left(\sum_{k=1}^{N}\left|z_{k}-w_{k}\right|^{2}\right)^{1 / 2}$, where $z=\left(z_{1}, \ldots, z_{N}\right)$ and $w=\left(w_{1}, \ldots, w_{N}\right)$. With respect to $d, B(z, r)$ will denote the open unit ball with center $z \in \mathbb{C}$ and radius $r$. Assume that $G$ is a domain in $\mathbb{C}^{N}$; that is, $G$ is a nonempty connected open subset of $\mathbb{C}^{N}$. By $H(G)$ it is denoted, as usual, the family of all holomorphic, or analytic, functions on $G$. The space $H(G)$ is a Fréchet space under the topology of uniform convergence on compact subsets of $G$. If $f \in H(G)$ and $\xi_{0} \in \partial G$, then we say that $f$ is holomorphically extendable through $\xi_{0}$ whenever there are $r>0$, $g \in H\left(B\left(\xi_{0}, r\right)\right)$ and a connected component $A$ of $G \cap B\left(\xi_{0}, r\right)$ such that $f=g$ in $A$. Throughout the pertinent literature, the fact that an $f \in H(G)$ is not holomorphically extendable through any $\xi \in \partial G$ is phrased with several synonymous sentences: $f$ is holomorphically nonextendable beyond $\partial G ; f$ is holomorphic exactly on $G$; $G$ is the domain of holomorphy of $f$; $\partial G$ is the natural boundary of $f$. The set of these functions will be denoted by $H_{e}(G)$.

If $N=1$ and $f \in H(G)$, one has that $f \in H_{e}(G)$ if and only if $R(f, a)=$ dist $(a, \partial G)$ for all $z \in G$, where $R(f, z)$ stands for the radius of convergence of the Taylor series of $f$ at $z$. If $f \in H_{e}(G)$, then $f$ has no holomorphic extension to any domain containing $G$ strictly, but the converse is not true (consider, for instance, $G:=\mathbb{C} \backslash(-\infty, 0]$ and $f:=$ the principal branch of $\log z)$. For $N=1$, both properties are equivalent if $G$ is a Jordan domain, in particular if $G=\mathbb{D}$. If $N>1$, we may have $H_{e}(G)=\varnothing$. In fact, the Cartan-Thullen theorem asserts that $H_{e}(G) \neq \varnothing$ if and only if $G$ is holomorphically convex (see for instance [197]). 
In 1933, Kierst and Szpilrajn [199] showed that $H_{e}(G)$ is residual in $H(G)$ for every domain $G \subset \mathbb{C}$. In 2000, Kahane [194 was able to extend this result to subspaces of $H(G)$. In fact, the same proof given in [194] allows us to weaken the hypotheses on the subspace $X$, so as to obtain (see 64]) the following assertion.

Theorem 2.31 (Kahane, 2000 [194]). Let $G \subset \mathbb{C}$ be a domain, and let $X$ be a Baire topological vector space with $X \subset H(G)$ such that the next conditions hold:

(a) All evaluation functionals $f \in X \mapsto f^{(k)}(a) \in \mathbb{C}\left(a \in G ; k \in \mathbb{N}_{0}\right)$ are continuous.

(b) For every $a \in G$ and every $r>\operatorname{dist}(a, \partial G)$ there exists $f \in X$ such that $R(f, a)<r$.

Then $X \cap H_{e}(G)$ is residual in $X$.

For instance, let $G=\mathbb{D}=\{z \in \mathbb{C}:|z|<1\}$, the open unit disc. For $0<p<\infty$ the Hardy space $H^{p}$ and the Bergman space $B^{p}$ are defined as the set $\{f \in H(\mathbb{D})$ : $\left.\|f\|_{p}<\infty\right\}$, where $\|f\|_{p}:=\sup _{0<r<1}\left(\int_{0}^{2 \pi}\left|f\left(r e^{i \theta}\right)\right|^{p} \frac{d \theta}{2 \pi}\right)^{1 / p}$ for $f \in H^{p},\|f\|_{p}:=$ $\left(\iint_{\mathbb{D}}|f(z)|^{p} \frac{d A(z)}{\pi}\right)^{1 / p}$ for $f \in B^{p}$, and $d A(z)$ denotes the normalized area measure on $\mathbb{D}$. They become F-spaces (i.e., completely metrizable topological vector spaces) with the distance $d(f, g)=\|f-g\|_{p}^{\alpha(p)}$, where $\alpha(p)=1$ if $p \geq 1(=p$ if $p<1)$. In fact, they are Banach spaces if $p \geq 1$. If $G \subset \mathbb{C}$ is a domain, consider the space $A^{\infty}(G)$ of holomorphic functions on $G$ having highly boundary-regular behavior; that is, $A^{\infty}(G):=\left\{f \in H(G): f^{(k)}\right.$ extends continuously to $\bar{G}$ for all $\left.k \geq 0\right\}$. It is also an F-space when it is endowed with the topology of uniform convergence of functions and all their derivatives on each compact set $K \subset \bar{G}$. Then $X \cap H_{e}(\mathbb{D})$ is residual in $X$ if $X=H^{p}, B^{p}$ or $A^{\infty}(\mathbb{D})$ (see 64, where further subspaces of $H(\mathbb{D})$ are studied in this respect). An explicit example of a nonextendable, very well-behaved function in the boundary is given in [249, Chap. 16]: the function

$$
f(z):=\sum_{n=0}^{\infty} a_{n} \exp (-\sqrt{n}) z^{n}, \text { where } a_{n}= \begin{cases}1 & \text { if } n \text { is a power of } 2 \\ 0 & \text { otherwise, }\end{cases}
$$

belongs to $A^{\infty}(\mathbb{D}) \cap H_{e}(\mathbb{D})$. More generally, let $G \subset \mathbb{C}$ be a regular domain, that is, it satisfies $G=\bar{G}^{0}$. In 1980, Chmielowski [123] discovered-as a consequence of an $N$-dimensional result (see also [260]) - that $A^{\infty}(G) \cap H_{e}(\mathbb{D}) \neq \varnothing$ for every such domain. By applying Theorem 2.31, one obtains the residuality of $A^{\infty}(G) \cap H_{e}(G)$ in $A^{\infty}(G)$ in this case; see $[75$.

2.6.2. Vector spaces of nonextendable functions. Plainly, the set $H_{e}(G)$ is not a linear space, so the study of its lineability makes sense. By using a clever, surprisingly easy approach, Aron, García and Maestre settled the problem for any space $H(G)$.

Theorem 2.32 (Aron, García, and Maestre, $2000[19]$ ). Let $G \subset \mathbb{C}^{N}$ be a domain of holomorphy, that is, $H_{e}(G) \neq \varnothing$. Then $H_{e}(G)$ is dense-lineable, spaceable, and algebrable in $H(G)$.

In fact, the closed infinite dimensional subspace $Y \subset H_{e}(G) \cup\{0\}$ obtained in [19] is $Y=\left\{f \in H(G): f\left(z_{n}\right)=0\right.$ for all $\left.n \in \mathbb{N}\right\}$, where $\left(z_{n}\right) \subset G$ is an adequate sequence. Hence $Y$ is also a closed infinitely generated algebra. Valdivia 266. shows that the dense subspace contained in $H_{e}(G)$ can be chosen to be nearly Baire. Recall that a locally convex space $E$ is called nearly Baire if, given a sequence $\left(A_{j}\right)$ of sum-absorbing balanced closed subsets covering $E$, there is $j_{0}$ such that $A_{j_{0}}$ 
is a neighborhood of 0 ; and a subset $A \subset E$ is said to be sum-absorbing whenever there is $\lambda>0$ such that $\lambda(A+A) \subset A$.

In [19] Aron et al. also considered the nonseparable Banach space $H^{\infty}:=\{f \in$ $H(\mathbb{D}): f$ is bounded on $\mathbb{D}\}$, endowed with the supremum norm. Then, with a similar idea, the authors of [19] proved that $\left(H^{\infty} \cap H_{e}(\mathbb{D})\right) \cup\{0\}$ contains an infinitely generated algebra that is nonseparable and closed in $H^{\infty}$. Hence $H^{\infty} \cap H_{e}(\mathbb{D})$ is spaceable and algebrable. In this special domain $\mathbb{D}$, a number of additional results have recently been obtained. For this, we consider properties (a)-(b) of Theorem 2.31 (for $G=\mathbb{D}$ ), as well as the following ones, where $X$ is a (topological) vector space:

(c) For every $f(z)=\sum_{n=0}^{\infty} a_{n} z^{n} \in X$, the function $\sum_{n \in Q} a_{n} z^{n} \in X$ for every $Q \subset \mathbb{N}_{0}$.

(d) Some denumerable subset of $H(\overline{\mathbb{D}})$ is a dense subset of $X$.

(e) $X \not \subset H(\overline{\mathbb{D}})$.

Here $H(\overline{\mathbb{D}})$ stands for the space of functions $f \in H(\mathbb{D})$ having holomorphic extension to some open set $\Omega_{f} \supset \overline{\mathbb{D}}$. Observe that properties (c) and (e) do not require any topological or algebraic structure on $X$.

Theorem 2.33 (Bernal, 2005 [64]). Assume that $X$ is a topological vector space with $X \subset H(\mathbb{D})$. We have:

(1) If $X$ is Baire metrizable and satisfies (a), (b), (c), and (d), then $X \cap H_{e}(\mathbb{D})$ is dense-lineable in $X$.

(2) If $X$ is metrizable, $X$ satisfies (d), and there is a subset of $X$ for which (c) and $(\mathrm{e})$ hold, then $X \cap H_{e}(\mathbb{D})$ is dense-lineable in $X$.

(3) If $X$ is Baire and satisfies (a), (b), and (c), then $X \cap H_{e}(\mathbb{D})$ is spaceable in $X$.

(4) If $X$ satisfies (a) and there is a subset of $X$ for which (c) and (e) hold, then $X \cap H_{e}(\mathbb{D})$ is spaceable in $X$.

Theorem 2.33, in whose proof Hadamard's lacunary theorem (see, e.g., [249, Chap. 16]) happens to be a main ingredient, applies successfully to the spaces $H^{p}, B^{p}$, and $A^{\infty}(\mathbb{D})$, among others. Turning to general domains in $\mathbb{C}$, the use of the Arakelian approximation theorem (see, e.g., 146]) leads us to an extension of Theorem 2.32 in which the growth of $f$ near each boundary point is as fast as prescribed.

Theorem 2.34 (Bernal, 2006 [66]). Let $G \subset \mathbb{C}$ be a domain and $\varphi: G \rightarrow(0,+\infty)$ a function. Then the set

$$
\left\{f \in H_{e}(G): \limsup _{z \rightarrow \xi} \frac{|f(z)|}{\varphi(z)}=+\infty \text { for all } \xi \in \partial G\right\}
$$

is spaceable and maximal-dense-lineable in $H(G)$.

Note that, in particular, $H_{e}(G)$ is always maximal-dense-lineable. Concerning subspaces of $H(G)$, it is easy to get lineability if not much more than mere nonvacuousness is assumed; in addition, the use of the Faber transform allowed the authors of 75 to obtain large closed manifolds of nonextendable boundary-regular function if $\partial G$ enjoys a soft structure.

Theorem 2.35 (Bernal, Calderón, and Luh, 2008 [75]). (a) Let $G \subset \mathbb{C}$ be a domain whose boundary does not contain isolated points, and let $X$ be a 
vector space over $\mathbb{C}$ with $X \subset H(G)$ satisfying $X \cap H_{e}(G) \neq \varnothing$ and $\{\varphi f$ : $f \in X\} \subset X$ for some nonconstant function $\varphi \in H(\bar{G})$. Then $X \cap H_{e}(G)$ is lineable.

(b) Assume that $G \subset \mathbb{C}$ is a Jordan domain with analytic boundary. Then $A^{\infty}(G) \cap H_{e}(G)$ is spaceable in $A^{\infty}(G)$.

In particular, $A^{\infty}(G) \cap H_{e}(G)$ is lineable if $G$ is regular. In 75 it is shown that if, in addition, $\mathbb{C} \backslash \bar{G}$ is connected and there is $M \in(0,+\infty)$ such that for any $a, b \in G$ there exists a curve $\gamma \subset G$ joining $a$ to $b$ for which length $(\gamma) \leq M$, then $A^{\infty}(G) \cap H_{e}(G)$ is dense-lineable. But, as shown in [267, the latter conditions are not at all necessary.

Theorem 2.36 (Valdivia, 2009 [267]). If $G \subset \mathbb{C}$ is a regular domain, then $A^{\infty}(G) \cap$ $H_{e}(G)$ is dense-lineable. In fact, there is a dense vector subspace $E$ in $A^{\infty}(G)$ such that $E \backslash\{0\} \subset H_{e}(G)$, and $E$ is nearly Baire.

In 267] the problem is posed as to whether "nearly Baire" can be replaced by "Baire".

2.6.3. A special case: strongly annular functions. Here we will deal with the special domain $G=\mathbb{D}$. One way of being nonextendable is to grow fast near the boundary. In this vein, an interesting family in $H(\mathbb{D})$ is $\mathcal{S} \mathcal{A}$, formed by the so-called strongly annular functions. By definition, a function $f \in H(\mathbb{D})$ belongs to $\mathcal{S} \mathcal{A}$ provided that

$$
\limsup _{r \rightarrow 1} \min \{|f(z)|:|z|=r\}=+\infty \text {. }
$$

Note that $\mathcal{S} \mathcal{A} \subset H_{e}(\mathbb{D})$. In 1975, Bonar and Carroll [96] established the residuality of $\mathcal{S} \mathcal{A}$. A lineability result is available.

Theorem 2.37 (Bernal and Bonilla, 2012 [71). $\mathcal{S A}$ is algebrable and maximal dense-lineable in $H(\mathbb{D})$.

In fact, weights can be imposed on the growth of functions; see [71. Turning to subspaces $X$ of $H(\mathbb{D})$, recall that some criteria for lineability properties of $X \cap$ $H_{e}(\mathbb{D})$ have been shown in the previous subsection. This raises the question of the lineability of the smaller set $X \cap \mathcal{S} \mathcal{A}$. But note that $\mathcal{S} \mathcal{A} \cap X=\varnothing$ for important spaces $X \subset H(\mathbb{D})$. For instance, it is evident that no $f \in \mathcal{S A}$ has a continuous extension to $\partial \mathbb{D}$; and $\mathcal{S} \mathcal{A} \cap H^{p}=\varnothing(p>0)$, thanks to the Fatou theorem asserting the existence of finite radial limit almost everywhere on $\partial \mathbb{D}$ for every $f \in H^{p}$. Nevertheless, the following positive result was provided by Redett [244]: $B_{\alpha}^{p} \cap \mathcal{S A} \neq \varnothing$ for every $p \in(0,+\infty)$ and every $\alpha \in(-1,+\infty)$, where $B_{\alpha}^{p}$ denotes the $\alpha$-weighted Bergman space, that is, the class of functions $f \in H(\mathbb{D})$ for which

$$
\|f\|_{p, \alpha}:=\left(\iint_{\mathbb{D}}|f(z)|^{p}(1-|z|)^{\alpha} \frac{d A(z)}{\pi}\right)^{\min \{1,1 / p\}}<+\infty .
$$

It becomes a separable F-space under the F-norm $\|\cdot\|_{p, \alpha}$. Note that $B_{0}^{p}=B^{p}$, the classical Bergman space. By exploiting Redett's approach in [244], a lineability assertion can be obtained.

Theorem 2.38 (Bernal and Bonilla, 2012 [71]). The set $\mathcal{S} \mathcal{A} \cap B_{\alpha}^{p}$ is dense-lineable in $B_{\alpha}^{p}$.

Problem 2.39. Is $X \cap \mathcal{S} \mathcal{A}$ spaceable/maximal dense-lineable for $X=B_{\alpha}^{p}$ (or even for other spaces $X \subset H(\mathbb{D}))$ ? 


\subsection{Miscellaneous.}

2.7.1. Annulling functions and sequences with finitely many zeros. A function $f \in$ $\mathcal{C}[0,1]$ is said to be an "annulling function" on $[a, b] \subset[0,1]$ if $f$ has infinitely many zeros in $[a, b]$ (see [137, Definition 2.1]). As we mentioned in Section 2.2.2, this set of annulling functions is spaceable (Enflo, Gurariy, and Seoane-Sepúlveda, [137. Corollary 3.8]). On a totally different framework, but related to the study of the amount of zeros of functions on a given interval, let us recall a question originally posed by Aron and Gurariy in 2003, where they asked whether there exists an infinite dimensional subspace of $\ell_{\infty}$, every nonzero element of which has only a finite number of zero coordinates. If we denote by $P$ the set of odd prime numbers and we call $x_{p}=\left(\frac{1}{p}, \frac{1}{p^{2}}, \frac{1}{p^{3}}, \frac{1}{p^{4}}, \ldots\right) \in \ell_{\infty}(p \in P)$, then it is easy to see that any nontrivial finite linear combination of $\left\{x_{p}: p \in P\right\}$ satisfies the desired property. Some partial answers to the original problem were recently given (for other sequence spaces) in [157] where the authors proved, among other results in this direction, the following:

Theorem 2.40 (García-Pacheco, Pérez-Eslava, and Seoane-Sepúlveda, 2010 [157]). Let $X$ be an infinite dimensional Banach space with a normalized Schauder basis $\left(e_{n}\right)_{n \in \mathbb{N}}$. There exists a linear space $V \subset X$ such that:

(a) If $a=\sum_{n=1}^{\infty} a[n] e_{n} \in V \backslash\{0\}$, then card $\{n \in \mathbb{N}: a[n]=0\}<\infty$.

(b) If $a, b \in V$, then $\sum_{n=1}^{\infty} a[n] b[n] e_{n} \in V$.

(c) $V$ is dense and not barrelled.

However, the question originally posed by Aron and Gurariy concerning spaceability for the $\ell_{\infty}$-case remains open.

2.7.2. The Denjoy-Clarkson property. It is well known that derivatives of functions of one real variable satisfy the Denjoy-Clarkson property: if $u: \mathbb{R} \rightarrow \mathbb{R}$ is everywhere differentiable, then the counterimage through $u^{\prime}$ of any open subset of $\mathbb{R}$ is either empty or has positive Lebesgue measure. Extending this result to several real variables is known as the Weil Gradient Problem [269] and, after being an open problem for almost 40 years, was finally solved (in the negative) for $\mathbb{R}^{2}$ by Buczolich in 2002 119. His example was later simplified by Deville and Matheron [131. They constructed an everywhere differentiable function on $Q=[0,1]^{n}$ and extended it through $\mathbb{Z}^{n}$-periodicity to the whole of $\mathbb{R}^{n}$, obtaining a bounded, everywhere differentiable function $f: \mathbb{R}^{n} \rightarrow \mathbb{R}$ such that

(1) $f$ and $\nabla f$ vanish on the boundary of $Q$,

(2) $\|\nabla f\|=1$ almost everywhere in $\mathbb{R}^{n}$ and $\|\nabla f(x)\| \leq 1$ for all $x \in \mathbb{R}^{n}$.

Thus it is clear that $f$ fails the Denjoy-Clarkson property, since $(\nabla f)^{-1}(B(0,1))$ is a nonempty set of zero Lebesgue measure. In [154] the authors proved that for every $n \geq 2$ there exists an infinite dimensional Banach space of differentiable functions on $\mathbb{R}^{n}$ which (except for 0 ) fail the Denjoy-Clarkson property.

2.7.3. Non-Lipschitz functions with bounded gradient. A standard result from real analysis states that, for any interval $I$, a differentiable function $f: I \longrightarrow \mathbb{R}$ is Lipschitz if and only if it has bounded derivative. One could think if the result still holds under weaker conditions. In [273] an example is provided of a continuous non-Lipschitz function, which is differentiable almost everywhere and has bounded 
derivative almost everywhere. Recently, Jímenez-Rodríguez, Muñoz, and SeoaneSepúlveda [190, Theorem 2.1, 3.1] proved the following results.

(a) The set of continuous functions on $[0,1]$ which are almost everywhere differentiable, with almost everywhere bounded derivative and not Lipschitz is $\mathfrak{c}$-lineable.

(b) The set of differentiable functions $f: \mathbb{R} \longrightarrow \mathbb{R}^{2}$ that do not enjoy the classical Mean Value Theorem is $\mathfrak{c}$-lineable.

(c) The set of differentiable functions $f: D \rightarrow \mathbb{R}$ with bounded gradient, nonLipschitz, and therefore not satisfying the classical Mean Value Theorem is c-lineable ([190, Theorem 3.1]), where

$D=\left\{(x, y) \in \mathbb{R}^{2}: x^{2}+y^{2}<1\right\} \backslash\left\{(x, y) \in \mathbb{R}^{2}: x=0\right.$ and $\left.y>0\right\}$,

which is a path-connected, nonconvex set.

We remark that the above results can be improved to dense-lineability by means of [20, Theorem 2.2 and Remark 2.5]. Also, just recently [189], item (a) above has been improved by showing that $c_{0}$ is isometrically isomorphic to a subspace of Cantor-Lebesgue functions, that is, continuous non-Lipschitz functions $f:[0,1] \rightarrow$ $\mathbb{R}$ with $f^{\prime}=0$ almost everywhere. This, in particular, gives spaceability in $\mathcal{C}[0,1]$ of the set defined in (a). Furthermore, Balcerzak et al. 31] have shown the dense strongly c-algebrability in $\mathcal{C}[0,1]$ of the smaller set of strongly singular functions. Recall that a function $f:[0,1] \rightarrow \mathbb{R}$ is called strongly singular if $f \in C B V[0,1]$ (the space of bounded variation continuous functions on $[0,1]), f^{\prime}=0$ almost everywhere and $f$ is not constant on any subinterval of $[0,1]$. The spaceability of the last set (with nonseparable subspace) in the space $C B V[0,1]$ (endowed with the norm $\|f\|=\sup _{[0,1]}+$ Variation $\left._{[0,1]} f\right)$ is also shown in [31].

Problem 2.41. Are any of the above sets from (b) or (c) algebrable?

2.7.4. Tamed entire functions and wild behavior near the boundary. In 1924, K. Grandjot [170] modified Mittag-Leffler's function to get an entire function $\mathbb{C} \rightarrow \mathbb{C}$ receding to 0 along any algebraic curve, so along any (straight) line. This surprising result has been improved in several ways, for instance, adding boundedness to all derivatives or imposing integrability on every line; see, e.g., [9, 275]. The question naturally arises as to whether this kind of "tamed" entire functions enjoys some sort of lineability. This task was started in the papers [58, 59], whose statements were improved by Armitage (2000 [1] ) and Bonilla (2002 [101]). The main result can be summarized as follows.

Theorem 2.42. Let $\alpha \in(0,+\infty)$, and let $\varphi:[0,+\infty) \rightarrow(0,+\infty)$ be an increasing function. There exists a vector subspace $M$ which is dense in $H(\mathbb{C})$ such that $\lim _{z \rightarrow \infty, z \in S} \exp \left(|z|^{\alpha}\right) f(z)=0$ for every strip or unbounded algebraic curve $S$ and every $f \in M$, and

$$
\lim _{r \rightarrow+\infty} \frac{\max \{|f(z)|:|z|=r\}}{\varphi(r)}=+\infty
$$

for every $f \in M \backslash\{0\}$.

Notice that one obtains entire functions being small and big simultaneously. Additional properties enjoying lineability as well can be found in the cited works [11,58, 59, 101]. A harmonic version is discussed in [100, where, in addition, they prove the dense-lineability in $\mathcal{H}(\mathbb{B})$ of the family of harmonic functions $f$ on the Euclidean unit ball $\mathbb{B}$ of $\mathbb{R}^{N}$ having zero nontangential limit at every point of $\partial \mathbb{B}$. 
Concerning domains different from $\mathbb{C}$, Bernal, Calderón, and Prado-Bassas 77 ] gave in 2004 a linear version of an old theorem due to Kierst and Szpilrajn [199. asserting the residuality of a family of functions holomorphic in $G$ having wild behavior near the boundary. Here $G$ is a Jordan domain in $\mathbb{C}$. Specifically, it is shown in 77 the dense-lineability in $H(G)$ of the set of holomorphic functions $f$ in $G$ satisfying that the cluster set $C(f, \gamma, \xi)$ of $f$ along $\gamma$ at each $\xi \in \partial G$ equals $\mathbb{C}_{\infty}$ for every $f \in M \backslash\{0\}$, every $\xi \in \partial G$ and every curve $\gamma \subset G$ tending to $\partial G$ whose closure does not contain $\partial G$. Recall that if $A \subset G$, then $C(f, A, \xi)$ is defined as the set $\left\{w \in \mathbb{C}_{\infty}: \exists\left(z_{n}\right) \subset A\right.$ such that $z_{n} \rightarrow \partial G$ and $\left.f\left(z_{n}\right) \rightarrow w\right\}$. An extension of this assertion to $L$-analytic functions on domains in $\mathbb{R}^{N}$, where $L$ is an elliptic operator can be found in 72 .

Some of the assertions above can be completed so as to include universality properties (see Section 3.2).

\section{HYPERCYCLIC MANIFOLDS}

In this section, we deal with a class of operators presenting chaotic dynamical behavior. The topic has been systematically studied during the last three decades.

3.1. Hypercyclicity and universality: examples and genericity. Traditionally, chaotic processes had been associated to nonlinear settings. Surprisingly, in 1929 Birkhoff 92 showed the existence of an entire function $\mathbb{C} \rightarrow \mathbb{C}$ whose sequence of translates $\{f(\cdot+a n): n \geq 1\}(a \in \mathbb{C} \backslash\{0\})$ approximates uniformly in compacta any prescribed entire function. This entails a rather wild dynamics for such a function $f$ under the action of a continuous linear self-mapping of $H(\mathbb{C})$, namely, the translation map $\tau_{a} g:=g(\cdot+a)$. In 1952, MacLane 218] demonstrated the same denseness property for the orbit $\left\{f^{(n)}: n \geq 1\right\}$ of some entire function $f$ under the action of the derivative operator $D g:=g^{\prime}$. From these prominent examples, and others that do not necessarily come from iterates of one self-mapping, many analysts have invested much effort in studying these kinds of phenomena, mostly during the last thirty years. The adequate abstract framework for these results is given in the next paragraph.

Let $X$ and $Y$ be two (Hausdorff) topological spaces and $T_{n}: X \rightarrow Y(n \in \mathbb{N}:=$ $\{1,2, \ldots\})$ be a sequence of continuous mappings. Then $\left(T_{n}\right)$ is said to be universal provided that there exists an element $x_{0} \in X$, called universal for $\left(T_{n}\right)$, such that the orbit $\left\{T_{n} x_{0}: n \in \mathbb{N}\right\}$ of $x_{0}$ under $\left(T_{n}\right)$ is dense in $Y$. We denote

$$
\mathcal{U}\left(\left(T_{n}\right)\right):=\left\{x \in X: x \text { is universal for }\left(T_{n}\right)\right\} .
$$

It is evident that the universality of some $\left(T_{n}\right)$ implies that $Y$ is separable. If $X$ and $Y$ are topological vector spaces and $\left(T_{n}\right) \subset L(X, Y):=\{$ continuous linear mappings $X \rightarrow Y\}$, then the words "universal" and "hypercyclic" are synonymous, although the term "hypercyclic" (coined by Beauzamy [55]) is mainly used to designate an operator (i.e., a continuous linear self-mapping $T \in L(X):=L(X, X))$ such that the sequence $\left(T^{n}\right)$ of its iterates is universal. We denote $H C(T):=$ \{hypercyclic vectors for $T\}=\mathcal{U}\left(\left(T^{n}\right)\right)$. Excellent surveys for the theory of hypercyclicity and universality are [52, [97, [173, [174, [176], and [202.

Under the last terminology, the previously mentioned theorems by Birkhoff and MacLane can be reformulated as follows: both translation and derivation operators are hypercyclic on the space $H(\mathbb{C})$ endowed with compact-open topology. In 1941 , 
Seidel and Walsh gave a non-Euclidean version of Birkhoff's theorem by showing that, if $H(\mathbb{D})$ is endowed with the compact-open topology, and

$$
C_{\varphi}: f \in H(\mathbb{D}) \mapsto f \circ \varphi \in H(\mathbb{D})
$$

denotes the composition operator generated by a non-Euclidean translation $\varphi(z)=$ $\frac{z+a}{1+\bar{a} z}(a \in \mathbb{D} \backslash\{0\})$, then $C_{\varphi}$ is hypercyclic. And in 1991, Godefroy and Shapiro [167] unified and strengthened the theorems by Birkhoff and MacLane in the following way: any $T \in L(H(\mathbb{C}))$ that is not a scalar multiple of the identity $I$ and that commutes with the derivative operator $D$ is hypercyclic. In particular, $P(D)$ is hypercyclic on $H(\mathbb{C})$ for every nonconstant polynomial $P$ with complex coefficients. In fact (see for instance [167]), an operator $T \in L(H(\mathbb{C}))$ commutes with $D$ if and only if it commutes with translations, and if and only if $T=\Phi(D)$ for some entire function $\Phi$ with exponential type, meaning that there are positive constants $A, B$ such that $|\Phi(z)| \leq A e^{B|z|}$ for all $z \in \mathbb{C}$. Recall that if $\Phi(z)=\sum_{n=0}^{\infty} a_{n} z^{n}$ in $\mathbb{C}$, then $\Phi(D) f:=\sum_{n=0}^{\infty} a_{n} f^{(n)}$ for all $f \in H(\mathbb{C})$.

In 1969, Rolewicz [246] provided the first example of a hypercyclic operator on a Banach space: if $X=c_{0}$ or $\ell_{p}(1 \leq p<\infty)$ and

$$
B:\left(x_{1}, x_{2}, x_{3}, \ldots\right) \mapsto\left(x_{2}, x_{3}, x_{4}, \ldots\right)
$$

is the backward shift on $X$, then any scalar multiple $\lambda B(|\lambda|>1)$ is hypercyclic. In the same paper he proves that in order that a topological vector space $X$ supports a hypercyclic operator, $X$ must be infinite dimensional. Rolewicz posed the problem of whether every separable infinite dimensional Banach space supports a hypercyclic operator. This was answered in the affirmative by Ansari [8], Bernal [60, and Bonet and Peris 99. In 99 the authors even proved that this result was valid for Fréchet spaces. Recall that an F-space is a completely metrizable topological vector space, while a Fréchet space is a locally convex F-space. Recently, Shkarin [259] has shown that every normed space of countable algebraic dimension supports a hypercyclic operator.

Another remarkable example in the setting of Banach spaces is the following. For $p \in[1,+\infty)$ consider the Hardy space $H^{p}$ on the open unit disc $\mathbb{D}$. The automorphisms (i.e., the bijective holomorphic self-mappings) of $\mathbb{D}$ are exactly the fractional linear mappings of the form $\varphi(z)=k \frac{z-a}{1-\bar{a} z}$. In Shapiro's book [255] it is proved that $C_{\varphi}$ is hypercyclic on $H^{p}$ if and only if $\varphi$ lacks fixed points in $\mathbb{D}$; see [115] and [147] for extensions. Turning to sequences of operators, Bernal and Montes 82 showed that the sequence $\left(C_{\varphi_{n}}\right)$ generated by a sequence $\left(\varphi_{n}\right)$ of automorphisms of $\mathbb{D}$ is universal on $H(\mathbb{D})$ if and only if $\sup _{n \geq 1}\left|\varphi_{n}(0)\right|=1$; moreover, if $\left\{\psi_{n}(z)=a_{n} z+b_{n}\right\}_{n \geq 1}$ is a sequence of automorphisms of $\mathbb{C}$, then $\left(C_{\psi_{n}}\right)$ is universal on $H(\mathbb{C})$ if and only if the sequence $\left\{\min \left\{\left|b_{n}\right|,\left|b_{n} / a_{n}\right|\right\}_{n \geq 1}\right.$ is unbounded. The reader can find extensions of these results to other domains and to other kinds of self-mappings in [82, [224], and [175]. Moreover, if $\left(\Phi_{n}(D)\right)$ is a sequence of differential operators, such that each $\Phi_{n}$ is an entire function with exponential type and there exist subsets $A, B \subset \mathbb{C}$ each of them with at least one finite accumulation point, and satisfying $\lim _{n \rightarrow \infty} \Phi_{n}(z)=0(z \in A)$ and $\lim _{n \rightarrow \infty} \Phi_{n}(z)=\infty(z \in B)$, then $\left(\Phi_{n}(D)\right)$ is hypercyclic on $H(\mathbb{C}) 62$.

Let us now consider the topological size of the set of universal vectors. From now on, $X$ and $Y$ will stand for topological vector spaces on the same field $\mathbb{K}(=\mathbb{R}$ or $\mathbb{C}$ ), and $T_{n}, T$ will be linear and continuous. Firstly, for a single hypercyclic operator $T \in L(X)$, since each member $T^{m} x_{0}$ of the orbit of a hypercyclic vector 
$x_{0} \in X$ is also hypercyclic (because $T$ has dense range), one obtains that $H C(T)$ is dense in $X$. Now, a sequence $\left(T_{n}\right) \subset L(X, Y)$ is said to be densely universal if $\mathcal{U}\left(\left(T_{n}\right)\right)$ is dense in $X$. If $Y$ is metrizable and separable and $\left(U_{k}\right)$ is a basis for the topology of $Y$, then

$$
\mathcal{U}\left(\left(T_{n}\right)\right)=\bigcap_{k \geq 1} \bigcup_{n \geq 1} T_{n}^{-1}\left(U_{k}\right),
$$

showing that $\mathcal{U}\left(\left(T_{n}\right)\right)$ is a $G_{\delta}$ subset. Thus, if in addition $X$ is a Baire space, we get that $\left(T_{n}\right)$ is densely universal if and only if $\mathcal{U}\left(\left(T_{n}\right)\right)$ is residual. Hence $\mathcal{U}\left(\left(T_{n}\right)\right)$ has large size in a topological sense. In particular, the property of being a hypercyclic vector is topologically generic as soon as $T$ is a hypercyclic operator on an F-space. The sequences of composition operators $\left(C_{\varphi_{n}}\right),\left(C_{\psi_{n}}\right)$ and of differential operators $\left(\Phi_{n}(D)\right)$ considered in the preceding paragraph (under the conditions specified there) are examples of densely universal sequences.

Several criteria guaranteeing large topological size for the family of universal vectors for $\left(T_{n}\right)$ - and even for subsequences of $\left(T_{n}\right)$-are known; see [176]. By $\left(n_{k}\right)$ we will denote a strictly increasing subsequence of $\mathbb{N}$. A sequence $\left(T_{n}\right) \subset L(X, Y)$ is said to be hereditarily universal if $\left(T_{n_{k}}\right)$ is universal for every $\left(n_{k}\right) \subset \mathbb{N}$, and hereditarily densely universal if $\left(T_{n_{k}}\right)$ is densely universal for every $\left(n_{k}\right) \subset \mathbb{N}$. Assume now that $X$ is an F-space and $Y$ is metrizable and separable. Then a sequence $\left(T_{n}\right) \subset L(X, Y)$ is said to satisfy the universality criterion (UC) provided that there are respective dense sets $X_{0} \subset X, Y_{0} \subset Y$ and a sequence $\left(n_{k}\right) \subset \mathbb{N}$ such that $T_{n_{k}} x \rightarrow 0$ for all $x \in X_{0}$ and, for every $y \in Y_{0}$, there is a sequence $\left(u_{k}\right) \subset X$ with $u_{k} \rightarrow 0$ and $T_{n_{k}} u_{k} \rightarrow y$. It can be proved (see [81] and [90]) that, for separable F-spaces $X$ and $Y$, the sequence $\left(T_{n}\right)$ satisfies the UC if and only if some subsequence of it is hereditarily densely universal. An operator $L \in L(X)$ is said to satisfy the hypercyclicity criterion (HCC) if the sequence of iterates $\left(T^{n}\right)$ satisfies the UC. It is elementary that $T$ is hypercyclic if $T$ is weakly mixing, meaning that $T \oplus T:(x, y) \in X \times X \mapsto(T x, T y) \in X \times X$ is hypercyclic. In 1992, Herrero [186] posed the problem of whether the reciprocal is true, and some years later León and Montes 208, raised the question of whether every hypercyclic operator satisfies the HCC. In 1999, Bès and Peris 90] proved that both problems are in fact equivalent. This question has been the "great open problem in hypercyclicity" for a long time, and it has served as a primary motivation for a decade-long development of the theory. Finally, de la Rosa and Read [130, settled the problem in the negative, and several examples of hypercyclic operators $T$ defined on classical spaces $X$ and not satisfying the HCC have been provided by Bayart and Matheron in [51].

For instance, if $\left(C_{\varphi_{n}}\right),\left(C_{\psi_{n}}\right),\left(\Phi_{n}(D)\right)$ are the sequences of operators considered in the fifth paragraph of this section, one has that each of the first two of them is hereditarily universal if and only if it is hereditarily densely universal, and this happens if and only if $\lim _{n \rightarrow \infty}\left|\varphi_{n}(0)\right|=1$ and $\lim _{n \rightarrow \infty} \min \left\{\left|b_{n}\right|,\left|b_{n} / a_{n}\right|\right\}=+\infty$, respectively. And $\left(\Phi_{n}(D)\right)$ is hereditarily densely universal if there are subsets $A, B$ satisfying the conditions specified above.

3.2. Hypercyclicity and lineability. After topological genericity has been analyzed, we study under what conditions the family of universal vectors enjoys algebraic genericity. It is evident that the set of universal vectors is never a vector space. 
3.2.1. Hypercyclity and dense-lineability. An extreme case of lineability is that in which $H C(T)=X \backslash\{0\}$. Observe that this happens if and only if $X$ admits no nontrivial proper closed $T$-invariant subset. If fact, C. Read [243] solved the invariant subset problem (analogous to the invariant subspace problem, connected with cyclic operators) for Banach spaces (it remains unsolved for Hilbert spaces) by exhibiting an operator $T$ on the sequence space $\ell_{1}$ for which any nonzero vector is hypercyclic. (Incidentally, in the recent paper [168] Goliński has given examples of operators $S$ without nontrivial proper invariant subspaces on classical non-Banach spaces $X$; hence the set of $S$-cyclic vectors is $X \backslash\{0\}$.)

But the last one is a very special operator. Let us go to a more general situation. In a chain of successive improvements, Herrero [185], Bourdon [114], Bès [87, and Wengenroth 270] demonstrated that if $T$ is a hypercyclic operator on an arbitrary topological vector space $X$, then $H C(T)$ is dense-lineable. In fact, they proved much more, and their results are contained in the following theorem.

Theorem 3.1. If $T$ is a hypercyclic operator on a topological vector space $X, \mathcal{P}$ is the family of all polynomials with coefficients in $\mathbb{K}$, and $x_{0}$ is a hypercyclic vector for $T$, then $M:=\left\{P(T) x_{0}: P \in \mathcal{P}\right\}$ is a dense $T$-invariant vector subspace of $X$ such that $M \backslash\{0\} \subset H C(T)$.

If $X$ is Banach, then $H C(T)$ is even maximal-dense-lineable 63, and the $T$ invariance is kept for the corresponding vector subspace. At this time, the maximaldense-lineability of $H C(T)$ for more general topological vector spaces seems to be unknown. It is also worth mentioning an important result by Grivaux [171] asserts that if $X$ is a Banach space and $\left(T_{j}\right)_{j \in \mathbb{N}}$ is a countable family of hypercyclic operators on $X$, then $\bigcap_{j=1}^{\infty} H C\left(T_{j}\right)$ is dense-lineable. She also proved in [171] that if $\left(T_{\lambda}\right)_{\lambda \in \Lambda}$ is a family of operators on a separable Fréchet space such that some $T_{\lambda_{0}}$ commutes with each $T_{\lambda}(\lambda \in \Lambda)$, then the set of common hypercyclic vectors $\bigcap_{\lambda \in \Lambda} H C\left(T_{\lambda}\right)$ is either empty or dense-lineable. As Bayart 44] showed, ven commutativity is not needed under adequate conditions.

As for sequences of linear mappings, it should be said that the mere residuality of the set of universal vectors does not entail lineability. For instance, let $\alpha=$ $\left(a_{k}\right) \in \mathbb{C}^{\mathbb{N}_{0}}$ be a sequence with $\lim \sup _{k \rightarrow \infty}\left|a_{k}\right|^{1 / k}<+\infty$, and define the associated diagonal operator $\Delta_{\alpha}$ as

$$
\Delta_{\alpha}: \sum_{k=0}^{\infty} f_{k} z^{k} \in H(\mathbb{C}) \mapsto \sum_{k=0}^{\infty} a_{k} f_{k} z^{k} \in H(\mathbb{C})
$$

Consider a sequence $\left\{\Delta_{\alpha_{n}}\right\}_{n \geq 1}$ of diagonal operators on $H(\mathbb{C})$, where $\alpha_{n}=$ $\left(a_{k, n}\right)_{k \geq 0}$. Then (see [78) $\left(\Delta_{\alpha_{n}}\right)$ is universal if and only if $\left\{\left(a_{k, n}\right)_{k \geq 0}: n \in \mathbb{N}\right\}$ is dense in $\mathbb{C}^{\mathbb{N}_{0}}$, in which case $\mathcal{U}\left(\left(\Delta_{\alpha_{n}}\right)\right)$ is residual; but no linear manifold contained in $\mathcal{U}\left(\left(\Delta_{\alpha_{n}}\right)\right) \cup\{0\}$ has dimension $\geq 2$.

Fortunately, lineability properties hold for the families of universal vectors of sequences of mappings under not too strong restrictions on the spaces and the mappings. In the following theorem a number of related results, starting from 1999, due to Bernal, Calderón, and Prado-Tendero [61,74, 85] are collected.

Theorem 3.2. Assume that $X, Y$ and $Y_{j}(j \in \mathbb{N})$ are topological vector spaces. We have the following: 
(a) If $Y$ is metrizable and $\left(T_{n}\right) \subset L(X, Y)$ is hereditarily universal, then $\mathcal{U}\left(\left(T_{n}\right)\right)$ is lineable. If, in addition, $X$ is metrizable and separable and $\left(T_{n}\right)$ is hereditarily densely universal, then $\mathcal{U}\left(\left(T_{n}\right)\right)$ is dense-lineable.

(b) Suppose that $X$ and the $Y_{j}$ 's are metrizable and separable, $X$ is Baire, $\left(T_{j, n}\right)_{n \geq 1} \subset L\left(X, Y_{j}\right)$ for each $j \in \mathbb{N}$, and each sequence $\left(T_{j, n}\right)_{n \geq 1}$ is hereditarily densely universal. Then the set $\bigcap_{j=1}^{\infty} \mathcal{U}\left(\left(T_{j, n}\right)_{n \geq 1}\right)$ of common universal vectors is dense-lineable.

Observe that the conclusions of Theorem 3.2(a) hold if there is a subsequence of $\left(T_{n}\right)$ that is hereditarily (densely) universal. Analogously, the conclusion of Theorem 3.2(b) remains valid if each $\left(T_{j, n}\right)_{n \geq 1}$ admits a subsequence (which may depend on $j$ ) being hereditarily densely universal.

For instance, the sets of functions which are respectively universal for the sequences $\left(C_{\varphi_{n}}\right),\left(C_{\psi_{n}}\right),\left(\Phi_{n}(D)\right)$ considered in Section 3.1-under the restrictions imposed there -are dense-lineable (see also 85. for combinations of composition operators with other kinds of operators). In particular, $\mathcal{U}\left(\left(\tau_{a_{n}}\right)\right)$ is dense-lineable provided that $\left(a_{n}\right)$ is an unbounded sequence in $\mathbb{C}$. With more sophisticated techniques it can be proved that for a fixed large set $A \subset \mathbb{C}$ (belonging to a wide class) the family of Birkhoff-universal entire functions presenting rapid decay along $A$ is dense-lineable; see [76] for details. In turn, the results of [76 complete some results due to Calderón [120] about universality and vanishing on strips. Hence rapid decay (see Subsection 2.7.4) is compatible with Birkhoff universality in order to generate lineability. Armitage [10] gives a direct proof of the existence of a dense vector subspace $M$ in the space $\mathcal{H}_{N}$ of all harmonic functions on $\mathbb{R}^{N}$ - endowed with the compact-open topology - such that each function $f \in M$ satisfies a prescribed growth condition and, if $f \neq 0$, it is universal with respect to the sequence of partial derivations of all orders. An explicit construction of a common dense vector space of hypercyclic vectors for a countable family of weighted backward shifts acting on a Banach space supporting a Schauder basis is given by Seoane-Sepúlveda in 254. As an application of Theorem 3.2(b) we have (see [74) that the family of holomorphic monsters created by Luh [217] in 1985 and investigated by GrosseErdmann (who proved its residuality, see [172]) is dense-lineable. If $G \subset \mathbb{C}$ is a domain, then a function $f \in H(G)$ is said to be a holomorphic monster whenever, for each derivative or antiderivative $F$ of $f$ of any order, each $g \in H(\mathbb{D})$ and each $\xi \in \partial G$ there exists a sequence $\left(\tau_{n}\right)$ of affine linear transformations with $\tau_{n}(z) \rightarrow \xi$ uniformly on $\mathbb{D}$ such that $\tau_{n}(\mathbb{D}) \subset G(n \in \mathbb{N})$ and $f\left(\tau_{n}(z)\right) \rightarrow g(z)$ compactly in $\mathbb{D}$.

Another outstanding example to which methods close to Theorem 3.2 can be applied is that of universal series. During the 1970s, Chui and Parnes [124 and Luh [216] provided a holomorphic function in the unit disc which is universal with respect to overconvergence. More precisely, they constructed a function $f(z)=$ $\sum_{n=0}^{\infty} a_{n} z^{n} \in H(\mathbb{D})$ satisfying that, given a compact set $K$ with connected complement and $K \cap \overline{\mathbb{D}}=\varnothing$, a function $g$ continuous on $K$ and holomorphic in its interior $K^{\circ}$, and $\varepsilon>0$, there is $n \in \mathbb{N}$ such that

$$
\left|\sum_{k=0}^{n} a_{k} z^{k}-g(z)\right|<\varepsilon \text { for all } z \in K .
$$

The topological generic nature of this property was shown in 1996 by Nestoridis 234] who, in fact, proved that $K$ can be allowed to meet $\partial \mathbb{D}$ (i.e., $K \cap \mathbb{D}=\varnothing$ ). In 
2005, Bayart 44 established the dense-lineability in $H(\mathbb{D})$ of the class of these functions $f$, which are known as universal Taylor series. Since 1996, many extensions of these results, for other domains and more restrictive classes of functions, have been performed (see the recent papers [30, 49, 53, 132, 228] and references therein). They can be put into a more general context. Let $X$ be a metrizable topological vector space over the field $\mathbb{K}=\mathbb{R}$ or $\mathbb{C},\left\{x_{n}\right\}_{n>0} \subset X$ be a fixed sequence, $\left\{e_{n}\right\}_{n \geq 0}$ be the canonical basis of $\mathbb{K}^{\mathbb{N}_{0}}$, and $A$ be a subspace of $\mathbb{K}^{\mathbb{N}_{0}}$ carrying a complete metrizable vector space topology. Assume that the coordinate projections $a=\left(a_{n}\right) \in A \mapsto a_{m} \in \mathbb{K}$ are continuous for any $m$ and that the set $\left\{a=\left(a_{n}\right) \in \mathbb{K}^{\mathbb{N}_{0}}:\left\{n: a_{n} \neq 0\right\}\right.$ is finite $\}$ is a dense subset of $A$. Then a sequence $a \in A$ is said to belong to the set $\mathcal{U}_{A}$ of restricted universal series in $A$ with respect to $\left(x_{n}\right)$ provided that, for every $x \in X$, there exists a sequence $\left(k_{n}\right) \subset \mathbb{N}_{0}$ such that

$$
\sum_{j=0}^{k_{n}} a_{j} x_{j} \rightarrow x \quad \text { and } \quad \sum_{j=0}^{k_{n}} a_{j} e_{j} \rightarrow a \quad \text { as } \quad n \rightarrow \infty .
$$

In [49, Bayart, Grosse-Erdmann, Nestoridis, and Papadimitropoulos characterized (and applied to a great deal of function spaces) the nonemptyness of $\mathcal{U}_{A}$, and proved that this is equivalent to its dense-lineability. Koumandos, Nestoridis, Smyrlis, and Stefanopoulos [204 have recently investigated under what conditions denselineability holds when $A=\bigcap_{p>1} \ell_{p}, \ell_{q}(1<q<\infty), c_{0}$ or $\mathbb{K}^{\mathbb{N}_{0}}$, endowed with their natural topologies; applications to trigonometric series in $\mathbb{R}^{\mathbb{N}}$ and Dirichlet series are furnished.

One can obtain dense-lineable sets of universal Taylor series satisfying, simultaneously, other universality properties. In particular, some kind of wild behavior near the boundary (see Subsection 2.7.4) is compatible with the property of being a universal Taylor series. For instance, Bernal, Bonilla, Calderón, and Prado-Bassas [73. showed in 2009 that the family of universal Taylor series $f$ having maximal (i.e., equal to $\left.\mathbb{C}_{\infty}\right)$ cluster set $C(f, \gamma, \xi)$ at each $\xi \in \partial \mathbb{D}$ along any curve $\gamma \subset \mathbb{D}$ tending to $\partial \mathbb{D}$ whose closure does not contain $\partial \mathbb{D}$ is dense-lineable in $H(\mathbb{D})$. Incidentally, in [80] it has been shown the maximal-dense-lineability in $H(G)$ of the class of functions $f \in \mathcal{U}\left(\left(C_{\varphi_{n}}\right)\right)$ satisfying that boundary property, where $G$ is a Jordan domain and $\left(C_{\varphi_{n}}\right)$, is the sequence of composition operators generated by adequate holomorphic self-mappings $\varphi_{n}: G \rightarrow G$.

3.2.2. Hypercyclicity and spaceability. Regarding large closed subspaces, the following assertion (see [83]) seems to be the first result in the setting of universality (if one disregards the aforementioned "extreme" example by Read 243]). Assume that $G \subset \mathbb{C}$ is a domain that is not conformally equivalent to $\mathbb{C} \backslash\{0\}$. Suppose also that $\left(\varphi_{n}\right)$ is a sequence of automorphisms of $G$ that is runaway, in the sense that, given a compact subset $K \subset G$, there exists $n \in \mathbb{N}$ such that $K \cap \varphi_{n}(K)=\varnothing$. Then, if the space of holomorphic functions $H(G)$ is endowed with the compact-open topology, the sequence $\left(C_{\varphi_{n}}\right)$ of composition operators defined on $H(G)$ satisfies that $H C\left(\left(C_{\varphi_{n}}\right)\right)$ is spaceable.

Montes stated the following criterium on existence of closed subspaces within the set of hypercyclic vectors. The proof is based on the construction of appropriate basic sequences. He proved his result in the setting of Banach spaces. For the universality criterion (UC), we refer the reader to the end of Section 3.1. 
Theorem 3.3 (Montes, 1996 [223]). If $X$ is a separable Banach space, $T$ is an operator on $X$ such that $\left(T^{n}\right)$ satisfies the $U C$ for some $\left(n_{k}\right) \subset \mathbb{N}$, and there is an infinite dimensional closed vector subspace $M$ of $X$ for which

$$
T^{n_{k}} x \rightarrow 0 \text { for all } x \in M,
$$

then $H C(T)$ is spaceable.

For instance, if $1 \leq p<\infty$ and $\varphi$ is an automorphism of $\mathbb{D}$ without fixed points, then $H\left(C_{\varphi}\right)$ is spaceable in $H^{p}$ [223]. Also, consider a weight, that is, a bounded sequence $w=\left(w_{n}\right) \subset \mathbb{K} \backslash\{0\}$, as well as its associated weighted backward shift $B_{w}: x=\left(x_{n}\right) \in \ell_{p} \mapsto\left(w_{n} x_{n+1}\right) \in \ell_{p}$. Then $H C\left(B_{w}\right)$ is spaceable provided that $\sup _{n \geq 1} \prod_{k=1}^{n}\left|w_{k}\right|=\infty$ and $\sup _{n \geq 1} \lim _{k \rightarrow \infty} \prod_{\nu=1}^{n}\left|w_{\nu+k}\right|<\infty$ [176. Chap. 10]. As a negative result, in 223 it is shown that the set $H C(T)$ for the Rolewicz operator $T=\lambda B(|\lambda|>1)$ is not spaceable, although $\left(T^{n}\right)$ satisfies the UC. León and Montes [207] studied the spaceability of $H C\left(B_{w}\right)$ on $\ell_{2}$, and recently Menet 222] has characterized it on $\ell_{p}$ and $c_{0}: H C\left(B_{w}\right)$ is spaceable if and only if $\sup _{n \geq 1} \inf _{k \geq 1} \prod_{\nu=1}^{n}\left|w_{\nu+k}\right|<\infty$.

González, León, and Montes characterized the spaceability of $H C(T)$ for Banach space operators satisfying the UC. Their findings can be summarized as follows. Recall that an operator $S$ is called Fredholm provided that $S$ has finite dimensional kernel and cofinite dimensional closed range.

Theorem 3.4 (González, León, and Montes, 2000 [169]). Let X be a complex separable Banach space, and let $T$ be an operator on $X$ such that $\left(T^{n}\right)$ satisfies the $U C$. Then the following are equivalent:

(a) $H C(T)$ is spaceable.

(b) There exists an increasing sequence $\left(n_{k}\right) \subset \mathbb{N}$ and an infinite dimensional closed subspace $M_{0}$ of $X$ such that $T^{n_{k}} x \rightarrow 0$ for all $x \in M_{0}$.

(c) There exists an increasing sequence $\left(m_{k}\right) \subset \mathbb{N}$ and an infinite dimensional closed subspace $M_{1}$ of $X$ such that $\sup _{k \geq 1}\left\|\left.T^{m_{k}}\right|_{M_{1}}\right\|<+\infty$.

(d) The essential spectrum $\sigma_{e}(T):=\{\lambda \in \mathbb{C}: \lambda I-T$ is not Fredholm $\}$ meets the closed unit disc $\overline{\mathbb{D}}$.

Complementary criteria of spaceability and nonspaceability of $H C(T)$ and $H C\left(\left(T_{n}\right)\right)$ were provided by León and Müller 209] in 2006 for Banach spaces, and recently by Ménet 222] in the setting of Fréchet spaces. Once it has been proved that a given topological vector space supports hypercyclic operators, the question of whether $H C(T)$ is spaceable for some $T$ among them arises naturally. In 1997, León and Montes 207] gave a positive answer for every (separable, infinite dimensional) Banach space. In 2006, Petersson [237] and Bernal [65] independently solved the question in the affirmative for Fréchet spaces admitting a continuous norm. But nonexistence of continuous norms may be allowed: Bès and Conejero [89] constructed an operator $T$ on $\omega:=\mathbb{K}^{\mathbb{N}}$ for which $H C(T)$ is spaceable. Finally, Menet 221] has been able to prove that the assertion holds for every separable infinite dimensional Fréchet space.

Theorem 3.3 has been improved and extended in several directions, for instance:

- The sequence of powers $\left(T^{n}\right)$ is replaced by a sequence of continuous linear mappings $\left(T_{n}\right)$.

- The arrival space is allowed to be different from $X$, that is, $\left(T_{n}\right) \subset L(X, Y)$.

- The space $X$ may be a Fréchet space, or even just an F-space. 
- It is possible to obtain spaceability for the set of common universal vectors of a countable family $\left\{\left(T_{j, n}\right)_{n \geq 1}: j \in \mathbb{N}\right\}$ of sequences of continuous linear mappings.

- The condition $T_{n_{k}} \rightarrow 0$ pointwise on $M$ can be replaced by the mere convergence.

These extensions can be found in the papers by Bonet, Martínez-Giménez, and Peris [98, Aron, Bès, León, and Peris [14], León and Müller [209], Petersson [237], Bernal [65], and Bonilla and Grosse-Erdmann [105]. Specifically, by combining the results and the approaches of the proofs in these papers (for this action, Sections $10.1,10.2,10.5$ and 11.4 of the book [176], as well of the mentioned equivalence for $\left(T_{n}\right)$ of satisfying the UC and of being densely hereditarily hypercyclic for some $\left(n_{k}\right) \subset \mathbb{N}$, will reveal very useful insights), we obtain the following assertion. Note that for sequences $\left(T_{n}\right)$ there is no need to introduce a subsequence $\left(n_{k}\right)$ since one may always pass to subsequences.

Theorem 3.5. Let $X$ be a separable F-space with a continuous norm, let $Y_{j}(j \in$ $\mathbb{N})$ be separable metrizable topological vector spaces, and let $\left(T_{j, n}\right)_{n \geq 1} \subset L\left(X, Y_{j}\right)$ $(j \in \mathbb{N})$. Suppose that the following holds:

(i) For every $j \in \mathbb{N},\left(T_{j, n}\right)_{n \geq 1}$ is hereditarily densely universal.

(ii) There exists an infinite dimensional closed vector subspace $M$ of $X$ such that the sequence $\left(T_{j, n} x\right)_{n \geq 1}$ converges in $Y_{j}$ for every $x \in M$ and every $j \in \mathbb{N}$.

Then $\bigcap_{j=1}^{\infty} \mathcal{U}\left(\left(T_{j, n}\right)_{n \geq 1}\right)$ is spaceable.

We furnish some examples, which may be interesting even in the case of a single operator or of one sequence of operators. In 2010, Shkarin 258] proved for the derivative operator $D$ that $H C(D)$ is spaceable in $H(\mathbb{D})$ (he also notes that the spectrum $\sigma(D)=\varnothing$, so Theorem 3.4 breaks down for Fréchet spaces). His proof does not rely on Theorem 3.5, but it can be extracted from this theorem (see [176. Example 10.13]). By the first result given in this subsection, the Birkhoff operator $\tau_{a}(a \neq 0)$ also enjoys spaceability for its family of hypercyclic functions; see [102] for a corresponding assertion in the space of harmonic functions on $\mathbb{R}^{N}$. More generally, Petersson [237] showed in 2006 the spaceability of $H C(\Phi(D))$ in $H(\mathbb{C})$, where $\Phi$ is an entire function of exponential type that is not a polynomial. Recently, Menet [222] has completed the Shkarin-Petersson results by proving that $H C(P(D))$ is also spaceable if $P$ is a nonconstant polynomial. If $\Omega$ is a domain in $\mathbb{C}$ and $\varphi, \psi$ are two automorphisms of $\Omega$ such that $C_{\varphi}, C_{\psi}$ are hypercyclic on $H(\Omega)$, then $H C\left(C_{\varphi}\right) \cap H C(\psi)$ is spaceable [176, Chap. 11]. By using Theorem 3.5, it is demonstrated in [70] the following result, which complements the corresponding "generic" one stated by Müller [227] in 2009: given a countable set $E \subset \partial \mathbb{D}$, the set of continuous functions $f$ on $\partial \mathbb{D}$ whose sequence $\left\{\left.S_{n} f\right|_{E}\right\}_{n \geq 1}$ of partial Fourier sums restricted to $E$ is dense in $\mathbb{C}^{E}$ is spaceable in the space $C(\partial \mathbb{D})$ of complex continuous functions on the unit circle; its maximal-dense-lineability is also shown.

There exist pairs of operators with spaceable sets of hypercyclic vectors, such that the set of their common hypercyclic vectors is not spaceable. For instance, consider the weights $w=\left(\frac{n+1}{n}\right)$ and $v=(2,2, \ldots)$, the associated weighted backward shifts $B_{w}, B_{v}$, and the product maps $T_{1}:=B_{w} \oplus B_{v}:(x, y) \in \ell_{2} \oplus \ell_{2} \mapsto\left(B_{w} x, B_{v} y\right) \in \ell_{2} \oplus$ $\ell_{2}, T_{2}:=B_{v} \oplus B_{w}$. In 14 it is shown that $H C\left(T_{1}\right)$ and $H C\left(T_{2}\right)$ are spaceable, but $H C\left(T_{1}\right) \cap H C\left(T_{2}\right)$ is not. In the opposite side, Bayart [45] (see also [176, Chap. 11]) 
furnished in 2005 some criteria guaranteeing the spaceability of the set of common hypercyclic vectors of an uncountable family of operators on a Fréchet space. A corresponding result for dense-lineability of an uncountable family of sequences of operators on a Banach space, under rather strong assumptions, was obtained by the same author in [44].

Turning to the universal Taylor series described at the end of the preceding subsection, Bayart 43 . established that the set of universal Taylor series (as described at the end of the preceding section) is spaceable in $H(\mathbb{D})$. A generalization of this result to simply connected domains was stated by Charpentier 122 in 2010. In fact, in [122] the Bayart et al. result [49] asserted the dense-lineability of $\mathcal{U}_{A}$ as soon as $\mathcal{U}_{A} \neq \varnothing$ is completed by showing that if $X$ is a Banach space, then $\mathcal{U}_{A}$ is spaceable as soon as $\mathcal{U}_{A} \neq \varnothing$. In 2011, Menet 220 has proved the assertion if $X$ is a Fréchet space admitting a continuous norm, and the same author [221] has recently shown the same result for Fréchet spaces admitting a continuous seminorm $p$ with $\operatorname{codim}(\operatorname{ker} p)=\infty$. In 73 and 80 , respectively, the combined properties of maximality of cluster sets in each boundary point with either universality of Taylor series or compositional universality in $H(G)$ considered there (where $G$ is a Jordan domain in $\mathbb{C}$ ) are proved to give spaceability. As a related result, in 79 . the authors provided a large family of classical operators (for instance, differential or composition operators) $T: H(G) \rightarrow H(G)$ (with $G$ a domain in $\mathbb{C}$ ) which satisfied that, for any subset $A \subset G$ that is not relatively compact in $G$, the set $\{f \in H(G): \overline{(T f)(A)}=\mathbb{C}\}$ is spaceable. Nevertheless, nothing seems to be known about the spaceability of the family of holomorphic monsters in $G$ considered in the preceding subsection.

3.2.3. Hypercyclicity and algebrability. In contrast with dense-lineability or spaceability, not much is known about algebrability in the framework of hypercyclicity. The derivative operator belongs to the short list of lucky operators.

Theorem 3.6 (Aron, Conejero, Peris, and Seoane-Sepúlveda, 2007 [16] and 17]). Consider the derivative operator $D: H(\mathbb{C}) \rightarrow H(\mathbb{C})$. Then there is a residual subset $M$ of $H(\mathbb{C})$ such that, for each $f \in M$, the algebra generated by $f$ is contained in $H C(D) \cup\{0\}$.

Nevertheless, the algebrability of $H C(D)$ is still unknown. For composition operators, the situation is even worse: the translation operator $\tau_{a}$ does not admit algebras contained, except for zero, in $H C\left(\tau_{a}\right)$ [17]: In fact, the same argument given in [17] shows that, for any domain $G \subset \mathbb{C}$ and any sequence $\left(\varphi_{n}\right)$ of holomorphic self-mappings of $G$, the set $H C\left(\left(C_{\varphi_{n}}\right)\right) \cup\{0\}$ does not contain any algebra.

On the positive side, Bayart and Matheron [52, Chap. 8] made the following observation, which is extracted from the approach of [17]: Let $X$ be an F-algebra and $T \in L(X)$. Assume that, for every pair $(U, V)$ of nonempty open sets in $X$, any open neighborhood $W$ of 0 in $X$ and any $m \in \mathbb{N}$, one can find $u \in U$ and $q \in \mathbb{N}$ such that $T^{q}\left(u^{j}\right) \in W$ for all $j<m$ and $T^{q}\left(u^{m}\right) \in V$. Then there is a residual subset $M$ of $X$ such that, for each $f \in M$, the algebra generated by $f$ is contained in $H C(T) \cup\{0\}$. This can be applied, for instance, to the Rolewicz operator $T:=2 B$ acting on $X:=\ell_{1}$ if $X$ is endowed with the convolution product $\left(a_{n}\right) *\left(b_{n}\right)=\left\{\sum_{k=0}^{n} a_{k} b_{n-k}\right\}_{n \geq 1}$.

3.3. Other kinds of linear chaos. Here we briefly focus our attention on weaker and stronger kinds of hypercyclicity, as well as on a related form of chaos. 
3.3.1. Supercyclicity and frequent hypercyclicity. In 1974, Hilden and Wallen [187] introduced the notion of supercyclicity. Let $X$ be a Hausdorff topological vector space over $\mathbb{K}=\mathbb{R}$ or $\mathbb{C}$. An operator $T \in L(X)$ is said to be supercyclic provided that there is a vector $x_{0} \in X$, called supercyclic for $T$, such that the projective orbit $\left\{\lambda T^{n} x_{0}: n \geq 0, \lambda \in \mathbb{K}\right\}$ of $x_{0}$ under $T$ is dense in $X$. Again, the separability of $X$ is necessary for supercyclicity. It is plain that every hypercyclic operator is also supercyclic. The backward shift $B$ on $\ell_{2}$ is an example of a supercyclic operator that is not hypercyclic. As in the hypercyclic case, it is immediate that the set $S C(T)$ of supercyclic vectors for $T$ is dense as soon as $T$ is supercyclic. If $X$ is a separable F-space and $\left(U_{k}\right)$ is an open basis for $X$, then

$$
S C(T)=\bigcap_{k \geq 1} \bigcup_{\substack{n \geq 1 \\ \lambda \in \mathbb{K} \backslash\{0\}}}\left(T^{n}\right)^{-1}\left(\lambda U_{k}\right),
$$

showing that if $T$ is supercyclic then $S C(T)$ is a dense $G_{\delta}$ subset. Hence we have topological genericity in this case and the question of the algebraic size of $S C(T)$ arises naturally. In contrast to the hypercyclic case, dense invariant supercyclic linear manifolds are not always available.

Theorem 3.7 (Herrero, 1991 [185]). Let $X$ be a complex Banach space, and let $T$ be a supercyclic operator on $X$. Then $S C(T) \cup\{0\}$ contains a T-invariant dense vector subspace if and only if the set of isolated points in $\sigma(T)$ that are not in $\sigma_{e}(T)$ is empty.

Moreover, with the same approach of [114] it can be proved that if $T$ is supercyclic and the adjoint $T^{*}$ lacks eigenvalues then $S C(T) \cup\{0\}$ contains a $T$-invariant dense vector subspace. As for large closed subspaces within $S C(T)$, there have been remarkable contributions due to Salas [251] and Montes and Salas 225] (see also [226]). The next two results show some similarity to Theorem 3.3 .

Theorem 3.8 (Salas, 1999 251]). Let $T$ be an operator on a complex separable Banach space $X$ satisfying the following:

(i) There exist an increasing sequence $\left(n_{k}\right) \subset \mathbb{N}$, dense subsets $Y, Z$ of $X$, and a mapping $S: Z \rightarrow Z$ such that $T S=$ identity on $Z$ in such a way that $\left\|T^{n_{k}} y\right\|\left\|S^{n_{k}} z\right\| \rightarrow 0$ for each $(y, z) \in Y \times Z$.

(ii) $0 \in \sigma_{e}(T)$.

Then $S C(T)$ is spaceable.

Theorem 3.9 (Montes and Salas, 2001 [225]). Let $T$ be an operator on a complex separable Banach space $X$ satisfying the following:

(i) There exist a strictly increasing sequence $\left(n_{k}\right) \subset \mathbb{N}$, a sequence $\left(\lambda_{k}\right) \subset$ $\mathbb{C} \backslash\{0\}$, dense subsets $Y, Z$ of $X$, and a mapping $S: Z \rightarrow Z$ such that $T S=$ identity on $Z$ in such a way that $\lambda_{k} T^{n_{k}} y \rightarrow 0$ for every $y \in Y$ and $\lambda_{k}^{-1} S^{n_{k}} z \rightarrow 0$ for every $z \in Z$.

(ii) The sequence $\left(\lambda_{k} \alpha^{n_{k}}\right)$ is bounded for some $\alpha \in \sigma_{e}(T)$.

Then $S C(T)$ is spaceable.

As a prominent example, a characterization of spaceability of $S C\left(B_{w}\right)$ in $\ell_{p}$ $(1 \leq p<\infty)$ in terms of the weights $w_{n}$ is provided in [225]: $S C\left(B_{w}\right)$ is spaceable if and only if there exist a strictly increasing sequence $\left(m_{j}\right) \subset \mathbb{N}$ and a sequence 
$(q(n)) \subset \mathbb{N}$ such that

$$
\liminf _{n \rightarrow \infty}\left(\limsup _{m_{j}>n} \frac{\prod_{i=1}^{n} w_{m_{j}-n+i}}{\min _{q \leq q(n)} \prod_{i=1}^{n} w_{q+i}}\right)=0 .
$$

In particular, for the unweighted backward shift $B$, the set $S C(B)$ is not spaceable. The last property is also true on $c_{0}$ (see the survey [226], in which one can find further examples).

In 2006, Bayart and Grivaux [4] introduced the following notion as a quantified, stronger form of hypercyclicity, connected to ergodic theory. An operator $T$ on a topological vector space $X$ is said to be frequently hypercyclic provided there exists a vector $x_{0} \in X$ such that

dens $\left\{n \in \mathbb{N}: T^{n} x_{0} \in U\right\}>0$ for every nonempty open subset $U$ of $X$.

In this case, $x_{0}$ is called a frequently hypercyclic vector for $T$, and the set of these vectors will be denoted by $F H C(T)$. Recall that if $A \subset \mathbb{N}$, then the lower density and the upper density of $A$ are defined respectively, as

$$
\underline{\operatorname{dens}}(A)=\liminf _{n \rightarrow \infty}(1 / n) \operatorname{card}(A \cap\{1, \ldots, n\})
$$

and

$$
\overline{\operatorname{dens}}(A)=\limsup _{n \rightarrow \infty}(1 / n) \operatorname{card}(A \cap\{1, \ldots, n\}),
$$

where card $(B)$ denotes the cardinality of the set $B$. It turns out that wide families of operators that were hypercyclic (including, as seen in Section 3.1 composition operators $C_{\varphi}$ on $H(\mathbb{D})$ with $\varphi$ an automorphism without fixed points, nonscalar differential operators $\Phi(D)$ on $H(\mathbb{C})$, and the Rolewicz operator $\lambda B$ with $|\lambda|>1$ on $c_{0}$ or $\ell_{p}, 1 \leq p<\infty$, among others) are also frequently hypercyclic; see [47, [48, [103], and [104]. But there are hypercyclic operators that are not frequently hyperyclic. An example of this is the weighted backward shift $B_{w}$ on $\ell_{2}$ with $w=\left(\left(1+n^{-1}\right)^{1 / 2}\right)$; see [47.

Let us consider now the topological size of $F H C(T)$. Even on F-spaces, there is not a result analogous to the hypercyclic case. In fact, the more popular operators (derivative operators, weighted backward shifts, composition operators) satisfy that their respective sets of frequent hypercyclic vectors are not residual; see [4] and 104. Nevertheless, by following the approach of Bourdon 114, we get that denselineability is kept for $F H C(T)$.

Theorem 3.10 (Bayart and Grivaux, 2006 [47]). Let $T$ be a frequently hypercyclic operator on a separable $\mathrm{F}$-space $X$. There is a dense $T$-invariant linear manifold $M$ of $X$, every nonzero vector of which is frequently hypercyclic for $T$.

In 47 the question was raised of whether-analogously to the case of simple hypercyclicity - the set $\bigcap_{k} F H C\left(T_{k}\right)$ is dense-lineable, where all $T_{k}(k \in \mathbb{N})$ are frequently hypercyclic operators on the same Banach space $X$.

Similar to mere hypercyclicity, the existence of large closed subspaces of frequently hypercyclic vectors calls for additional assumptions. According to Bayart and Grivaux [47] - and introducing a modified version due to Bonilla and GrosseErdmann [104] - we say that an operator $T$ on a separable F-space $X$ satisfies the frequent hypercyclicity criterion (FHCC) if there is a dense subset $X_{0}$ of $X$ and a map $S: X_{0} \rightarrow X_{0}$ such that, for any $x \in X_{0}, T S x=x$ and both series $\sum_{n=1}^{\infty} T^{n} x, \sum_{n=1}^{\infty} S^{n} x$ converge unconditionally. Recall that a series $\sum_{n=1}^{\infty} x_{n}$ in 
an F-space $X$ converges unconditionally if for every $\varepsilon>0$ there is some $N \in \mathbb{N}$ such that $\left\|\sum_{n \in F} x_{n}\right\|<\varepsilon$ for every finite subset $F \subset\{N+1, N+2, \ldots\}$. Here $\|\cdot\|$ is an F-norm on $X$. If $T$ satisfies the FHCC, then $T$ is frequently hypercyclic, and in fact the FHCC is a powerful tool to check the property for the main kinds of operators.

Theorem 3.11 (Bonilla and Grosse-Erdmann, 2012 [105]). Let $T \in L(X)$, where $X$ is a separable F-space with a continuous norm. Suppose that

(i) $T$ satisfies the $F H C C$, and

(ii) there exists an infinite dimensional closed subspace $M_{0}$ of $X$ such that $T^{n} x \rightarrow 0$ for all $x \in M_{0}$.

Then the set $F H C(T)$ is spaceable.

As a nice application, Bès 88 has proved that, if $G \subset \mathbb{C}$ is a simply connected domain and $\varphi: G \rightarrow G$ is a univalent holomorphic function without fixed points, then the set $F H C\left(C_{\varphi}\right)$ is spaceable.

3.3.2. Distributional chaos. Finally, we deal with a related notion of chaos presenting some similarities with that of hypercyclicity, but revealing deep differences at the same time. The notion was introduced in 1994 by Schweizer and Smítal [256]. According to [256], if $X$ is a metric space with distance $d$, a continuous map $T: X \rightarrow X$ is said to be distributionally chaotic if there exist an uncountable set $\Gamma \subset X$ and $\varepsilon>0$ such that for every $\tau>0$ and each pair of distinct points $x, y \in \Gamma$, we have that $\overline{\operatorname{dens}}\left\{n \in \mathbb{N}: d\left(T^{n} x, T^{n} y\right)<\tau\right\}=1$ and $\underline{\operatorname{dens}}\left\{n \in \mathbb{N}: d\left(T^{n} x, T^{n} y\right)<\varepsilon\right\}=0$. Inspired by this concept and by the notion of irregular vector coined by Beauzamy [56] (if $X$ is a Banach space and $x_{0} \in X$, an operator $T \in L(X)$ is called irregular, provided that the sequence $\left(T^{n} x_{0}\right)$ is unbounded but it has a subsequence tending to 0 ), the authors of [57 presented the following concept. If $X$ is a Banach space and $T \in L(X)$, then a vector $x_{0} \in X$ is said to be distributionally irregular if there are increasing sequences $A=\left(n_{k}\right), B=\left(m_{k}\right) \subset \mathbb{N}$ such that

$$
\overline{\operatorname{dens}}(A)=\overline{\operatorname{dens}}(B)=1, \lim _{k}\left\|T^{n_{k}} x_{0}\right\|=0 \text {, and } \lim _{k}\left\|T^{m_{k}} x_{0}\right\|=\infty .
$$

We denote by $D I(T)$ the set of distributionally irregular vectors for $T$. If $D I(T) \neq$ $\varnothing$, then $T$ is distributionally chaotic [57, but the truth of the converse is unknown at this time. A number of lineability results have been obtained in [57]. The next theorem gathers a selection of them.

Theorem 3.12 (Bermúdez, Bonilla, Martínez-Giménez, and Peris, 2011 [57]).

(a) Assume that $X$ is a Banach space, that $T \in L(X)$, and that there exists a dense subset $X_{0} \subset X$ such that $\lim _{n \rightarrow \infty} T^{n} x=0$. Then $D I(T)$ is denselineable if at least one of the following conditions hold:

(i) $D I(T) \neq \varnothing$.

(ii) There exist an increasing sequence $A=\left(n_{k}\right) \subset \mathbb{N}$ and a vector $y \in X$ satisfying $\overline{\operatorname{dens}}(A)=1$ and $\lim _{k \rightarrow \infty}\left\|T^{n_{k}} y\right\|=\infty$.

(iii) There exists an increasing sequence $A=\left(n_{k}\right) \subset \mathbb{N}$ with $\overline{\operatorname{dens}}(A)=1$ and $\sum_{n=1}^{\infty} \frac{1}{\left\|T_{n}\right\|}<\infty$.

(b) If $X$ is infinite dimensional and separable, there exists a hypercyclic and distributionally chaotic operator $T$ such that $D I(T)$ is dense-lineable. 
Recently, the authors of 219] have constructed a hypercyclic operator $T$ and a nonhyperyciclic operator $S$ such that every nonzero vector is distributionally irregular for each of them. Concerning Beauzamy's irregular vectors, in [86] Bernardes et al. have proved, among other results, that if $X$ is a Fréchet space, $T \in L(X)$, and $T$ admits an irregular vector, then the set of irregular vectors is dense-lineable.

Several corresponding results for strongly continuous semigroups (also called $C_{0^{-}}$ semigroups) $\left(T_{t}\right)_{t \geq 0}$ - to which the notions of distributionally irregular vectors and distributional chaos can be extended in a natural way, as well as the notions of hypercyclicity and frequent hypercyclicity - are provided in [6] by Albanese, Barrachina, Mangino, and Peris. The study of lineability for the set of hypercyclic vectors for a $C_{0}$-semigroup is virtually closed since Conejero, Müller, and Peris 129. proved in 2007 that if $X$ is an F-space, then $\left(T_{t}\right)_{t \geq 0}$ is hypercyclic if and only if each $T_{t_{0}}\left(t_{0}>0\right)$ is hypercyclic, and if and only if some $T_{t_{0}}\left(t_{0}>0\right)$ is hypercyclic. In this case, the set of hypercyclic vectors for $\left(T_{t}\right)$ equals $H C\left(T_{t_{0}}\right)$ for any $t_{0}>0$. The assertion holds if one replaces hypercyclicity by frequent hypercyclicity. Turning to distributional chaos, it seems that reasonable criteria for the spaceability of $D I(T)$ have not been furnished up to the time of this writing.

\section{Zeros of POLYNOMials in BANACH SPACES}

First of all, let us recall the definition of polynomial on a Banach space $X$. By $B_{X}$ we denote the open unit ball of $X$.

Definition 4.1. Let $n \in \mathbb{N}$. A function $P: X \rightarrow \mathbb{K}$ is said to be an $n$-homogeneous polynomial if there is a continuous $n$-linear mapping $A: X \times \cdots \times X \rightarrow \mathbb{K}$ such that $P(x)=A(x, \ldots, x)$ for all $x \in X$. A polynomial on $X$ is a finite sum of homogeneous polynomials.

Note that we will only deal with continuous $n$-homogeneous polynomials. The usual notation for the space of $n$-homogeneous polynomials on $X$ is $\mathcal{P}\left({ }^{n} X\right)$, while $\mathcal{L}\left({ }^{n} X\right)$ denotes the space of continuous $n$-linear functionals on $X$. Both spaces are Banach spaces when endowed with the respective natural norms,

$$
\|P\|=\sup _{x \in B_{X}}|P(x)|, \quad\|A\|=\sup _{x_{1}, \ldots, x_{n} \in B_{X}}\left|A\left(x_{1}, \ldots, x_{n}\right)\right| .
$$

To each $n$-homogeneous polynomial $P$ there corresponds a unique symmetric $n$ linear mapping $A$. Let $\mathcal{L}_{s}\left({ }^{n} X\right)$ denote the space of such symmetric $n$-linear functionals. By the polarization formula, given an $n$-homogeneous polynomial $P$, one can recover the associated $A \in \mathcal{L}_{s}\left({ }^{n} X\right)$,

$$
A\left(x_{1}, \ldots, x_{n}\right)=\frac{1}{n ! 2^{n}} \sum_{\varepsilon_{j}= \pm 1, j=1, \ldots, n} \varepsilon_{1} \cdots \varepsilon_{n} P\left(\varepsilon_{1} x_{1}+\cdots+\varepsilon_{n} x_{n}\right) .
$$

Moreover, $\|P\| \leq\|A\| \leq \frac{n^{n}}{n !}\|P\|$. We refer the interested reader to [12, 134, 229, 230] for a full explanation of these results, as well as many others on polynomials defined on infinite dimensional spaces.

The study of the zeros of polynomials on complex spaces, due its fundamental nature, has an old origin dating at least from the 1950s (see, e.g., 24 for some references to earlier work). Nowadays, this investigation can be approached with tools of algebraic geometry, complex analysis, and functional analysis ([177, 183, 238]). The case of polynomials on $\mathbb{C}^{n}$ has been widely investigated, but the case of polynomials on infinite dimensional Banach spaces seems to be an even richer source of 
challenging questions. The following well-known result of Plichko and Zagorodnyuk can be viewed as a starting point for in the infinite dimensional setting:

Theorem 4.2 (Plichko and Zagorodnyuk, 1998 [238]). If $X$ is an infinite dimensional complex Banach space and $P$ is an n-homogeneous polynomial on $X$, then $P^{-1}(0)$ contains an infinite dimensional subspace $Y$.

However for real scalars the situation is radically different as the polynomial $P: \ell_{2} \rightarrow \mathbb{R}$ given by

$$
P(x)=\sum_{j=1}^{\infty} x_{j}^{2}
$$

shows. Even in the finite dimensional case, the field, $\mathbb{R}$ or $\mathbb{C}$, makes a big difference. Indeed, for the 2-homogeneous polynomial $P: \mathbb{C}^{n} \rightarrow \mathbb{C}, P(z)=z_{1}^{2}+\cdots+z_{n}^{2}$, we have that $P^{-1}(0)$ contains a vector space of dimension $\left[\frac{n}{2}\right]$, since the span of $\left\{e_{1}+i e_{2}, e_{3}+i e_{4}, \ldots\right\} \subset P^{-1}(0)$, where $e_{1}=(1,0,0, \ldots, 0), e_{2}=(0,1,0,0, \ldots, 0)$ and so on. The reader can observe that nothing interesting can be said for $P^{-1}(0)$ when $\mathbb{K}=\mathbb{R}$. As the following theorem reveals, this example is, in fact, illustrative of the general situation in the case $\mathbb{K}=\mathbb{C}$. For works related to the study of zeros of real polynomials, we refer to [15,21, 142, 143] and the references therein.

Theorem 4.3 (Plichko and Zagorodnyuk, 1998 [238]). Let X be a complex Banach space. Given positive integers $n$ and $k$, there is an integer $m(n, k) \in \mathbb{N}$ such that, whenever $\operatorname{dim}(X)=k$ and $P: X \rightarrow \mathbb{C}$ is an n-homogeneous polynomial, the set $P^{-1}(0)$ contains a subspace of dimension at least $m(n, k)$. Moreover, $m(n, k) \rightarrow \infty$ as $k \rightarrow \infty$.

To give an idea of how things work in the subject of polynomials in Banach spaces, we shall now provide a proof (obtained from [26] and from its generalization by Lourenço and Tocha in [215]) that follows exactly the same lines as that of [238, and which is done by induction on the homogeneity $n$. We shall exhibit the situation for $n=1,2$, and 3. Clearly, $m(1, k)=k-1$. The general case, on the other hand, will require the following well-known fact from the theory of several complex variables: if $f: \mathbb{C}^{k} \rightarrow \mathbb{C}$ is a holomorphic (complex analytic) function in $k \geq 2$ variables, then $f^{-1}(0)$ is either empty or an unbounded set.

$\underline{n=2}$ Let us now focus on 2-homogeneous polynomials. Let $P: X \rightarrow \mathbb{C}$ be a 2-homogeneous polynomial, with corresponding symmetric bilinear form $A: X \times X \rightarrow \mathbb{C}$. As we saw previously, there exists $x_{1} \neq 0$ such that $P\left(x_{1}\right)=0$. Consider $S=\left\{x \in X: A\left(x, x_{1}\right)=0\right\}$. Then $S$ is a subspace of $X$ having dimension at least $k-1$. Since $x_{1} \in S$, we can write $S=S_{1} \bigoplus\left[x_{1}\right]$. Provided that $\operatorname{dim}\left(S_{1}\right) \geq 2$, we can find $x_{2} \in S_{1} \backslash\{0\}$ with $P\left(x_{2}\right)=0$. For any $x \in \operatorname{span}\left\{x_{1}, x_{2}\right\}$,

$$
\begin{aligned}
P(x) & =P\left(a_{1} x_{1}+a_{2} x_{2}\right)=A\left(a_{1} x_{1}+a_{2} x_{2}, a_{1} x_{1}+a_{2} x_{2}\right) \\
& =a_{1}^{2} P\left(x_{1}\right)+2 a_{1} a_{2} A\left(x_{1}, x_{2}\right)+a_{2}^{2} P\left(x_{2}\right)=0,
\end{aligned}
$$

using the fact that $A$ is symmetric and that $x_{2} \in S_{1} \subset S$. Thus, provided $\operatorname{dim}(X) \geq 4$, every complex-valued 2-homogeneous polynomial on $X$ vanishes on a 2 dimensional subspace. It is clear that the argument continues, giving that every 2-homogeneous polynomial vanishes on a $\left\lfloor\frac{k}{2}\right\rfloor$ dimensional subspace of $X$. 
$\underline{n=3}$ For the case of 3 -homogeneous polynomials $P$, first take $x_{1} \in X \backslash\{0\}$ such that $P\left(x_{1}\right)=0$. Let $A: X \times X \times X \rightarrow \mathbb{C}$ be the associated symmetric 3 -linear form. Thus, we can write $X=\left[x_{1}\right] \bigoplus Y_{k-1}$, where $\operatorname{dim}\left(Y_{k-1}\right)=$ $k-1$. Now, consider the subspace $S\left(x_{1}, x_{1}\right)=\left\{x \in Y_{k-1}: A\left(x_{1}, x_{1}, x\right)=\right.$ $0\}$, which is a $(k-2)$ dimensional subspace. We can now write $X=$ $\left[x_{1}\right] \bigoplus S\left(x_{1}, x_{1}\right) \bigoplus\left[y_{1}\right]$, where $y_{1} \in Y_{k-1} \backslash S\left(x_{1}, x_{1}\right)$. By the above $n=2$ case, there is an $\left\lfloor\frac{k-2}{2}\right\rfloor$ dimensional subspace $S\left(x_{1}\right)$ of $S\left(x_{1}, x_{1}\right)$ on which the 2-homogeneous polynomial $x \mapsto A\left(x_{1}, x, x\right)$ vanishes. If it happens that $\left\lfloor\frac{k-2}{2}\right\rfloor \geq 2$ (i.e., if $k \geq 6$ ), then $\operatorname{dim}\left(S\left(x_{1}\right)\right) \geq 2$, and so there is a nonzero vector $x_{2} \in S\left(x_{1}\right)$ such that $P\left(x_{2}\right)=0$. It is a routine verification that $\left[x_{1}, x_{2}\right]$ is then contained in $P^{-1}(0)$. By now, the method of proof should be reasonably evident, and in fact one gets $m(3, k) \geq \max \{n: k \geq$ $\left.2^{n-1}(n+1)\right\}$.

$\underline{n>3}$ Clearly, the same argument can be applied in order to extend the above constructions to all homogeneities $n$ of $P$.

Corollary 4.4. Let $P: \mathbb{C}^{k} \rightarrow \mathbb{C}$ be an arbitrary (not necessarily homogeneous) polynomial of degree $n$. Then there is a subspace $V \subset \mathbb{C}^{k}$ whose dimension depends only on $k$, such that $\operatorname{dim}(V) \rightarrow \infty$ as $k \rightarrow \infty$, satisfying the condition $\left.P\right|_{V} \equiv P(0)$.

Having in mind the polynomials of the form $\sum x_{j}^{2}$, the case of real polynomials needs a special approach where odd homogeneous polynomials and evenhomogeneous polynomials are investigated by different fronts.

For the case of odd homogeneous polynomials we present here a result by R. Aron and P. Hájek.

Theorem 4.5 (Aron and Hájek 2006 24]). Let $P: \mathbb{R}^{N} \rightarrow \mathbb{R}$ be an $n$-homogeneous polynomial, where $n$ is odd. Let $k \in \mathbb{N}$. Then there is a subspace $X \subset \mathbb{R}^{N}, \operatorname{dim} X=$ $k$, such that $\left.P\right|_{X} \equiv 0$ provided $N$ is so big that

$$
N>k !\left(\log _{2} N\right)^{k}\left(\begin{array}{c}
k+n-1 \\
k-1
\end{array}\right) .
$$

Regarding the case of 2-homogeneous polynomial on a real Banach space $X$, let us recall that a 2 -homogeneous polynomial $P: X \rightarrow \mathbb{R}$ is said to be positive definite if $P(x) \geq 0$ for every $x \in X$ and $P(x)=0$ only for $x=0$. It is known (see [139]) that the following statements are equivalent:

(i) $X$ admits a positive definite 2-homogeneous polynomial.

(ii) There is a continuous injection of $X$ into a Hilbert space.

(iii) There is a 2-homogeneous polynomial $P$ on $X$ whose set of zeros is contained in a finite dimensional subspace of $X$.

In particular, there are real positive definite 2-homogeneous polynomials on $X$ whenever $X$ is separable or a $\mathcal{C}(K)$ space for some compact, separable $K$. On the other hand, $c_{0}(\Gamma)$ and $\ell_{p}(\Gamma), p>2$, do not admit positive definite 2-homogeneous polynomials. The following result is proved via an interesting use of Zorn's Lemma:

Theorem 4.6 (Aron, Boyd, Ryan, and Zalduendo, 2003 [15]). Let $X$ be a real Banach space which does not admit a positive definite 2-homogeneous polynomial. Then, for every 2-homogeneous polynomial $P: X \rightarrow \mathbb{R}$, there is an infinite dimensional subspace of $X$ on which it is identically zero. 
Theorem 4.5] shows that given any $k \in \mathbb{N}$ and any odd positive integer $n$, there is some $N \in \mathbb{N}$ such that for any $n$-homogeneous polynomial $P: \mathbb{R}^{N} \rightarrow \mathbb{R}$, there is a $k$-dimensional subspace of $\mathbb{R}^{N}$ on which $P$ is identically 0 . From this, it seems a reasonable question to wonder whether something "better" happens if we replace $\mathbb{R}^{N}$ by an infinite dimensional real Banach space $X$ :

Given any odd $n$ and any $n$-homogeneous polynomial $P: X \rightarrow \mathbb{R}$, is there an infinite dimensional subspace $Y \subset X$ such that $\left.P\right|_{Y} \equiv 0$ ?

The answer is actually no. In fact, one has the following:

Theorem 4.7 (Aron and Hájek, 2007 [23]). Given any real, separable, infinite dimensional Banach space $X$ and any odd $n \in \mathbb{N}$, there is an $n$-homogeneous polynomial $P: X \rightarrow \mathbb{R}$ such that $P^{-1}(0)$ does not contain an infinite dimensional subspace.

From Theorem 4.2 a natural step forward is, How "big" is $Y$ ? In view of the previous results, the following questions arise naturally:

(Q1) Suppose that the complex Banach space $X$ is nonseparable. Does it follow that there is a nonseparable subspace contained in $P^{-1}(0)$ ?

(Q2) For a real Banach space $X$ and an $n$-homogeneous polynomial $P: X \rightarrow$ $\mathbb{R}$, are there situations where $P^{-1}(0)$ does contain an infinite dimensional subspace?

(Q3) Let $P: X \rightarrow \mathbb{K}$ be an $n$-homogeneous polynomial, where $\mathbb{K}=\mathbb{R}$ or $\mathbb{C}$, and let $M, N \subset X$ be two maximal subspaces of $P^{-1}(0)$. What can be said about the relation between $M$ and $N$ ? ( $M \subset X$ is a maximal subspace of $P^{-1}(0)$ means that $M$ is a vector subspace of $P^{-1}(0)$ that is not strictly contained in any larger subspace $P^{-1}(0)$.)

Let us now provide some answers to each of these questions. Below, $(\mathrm{A} j)$ denotes the answer for the $(\mathrm{Q} j)$ above.

(A1) The answer is affirmative for $\ell_{\infty}$. To be more precise, we have:

Theorem 4.8 (Fernández-Unzueta, 2006 [140]). Let E be a complex Banach space containing $\ell_{\infty}$. For every $n$, every $n$-homogeneous polynomial $P: E \rightarrow \mathbb{C}$ vanishes on a nonseparable subspace of $E$.

In addition, in [140] it is also shown that in the case of real $\ell_{\infty}$, if $P: \ell_{\infty} \rightarrow \mathbb{R}$ vanishes on a copy of $c_{0}$, then $P \equiv 0$ on a nonseparable subspace.

On the other hand, and responding to a question of [34] (see also [15]), Avilés and Todorcevic [28] have shown that the general answer to question (Q1) is no.

Theorem 4.9 (Avilés and Todorcevic, 2009 [28]). There is a 2-homogeneous polynomial $P: \ell_{1}\left(\aleph_{1}\right) \rightarrow \mathbb{C}$ such that $P^{-1}(0)$ contains no nonseparable subspace.

(A2) Concerning the second question, partial information has already been noted in Theorems 4.5. 4.6. and 4.7. Let us assume that $P: X \rightarrow \mathbb{R}$ is a 2homogeneous polynomial on a nonseparable Banach space $X$. In this case, there is some additional, albeit partial, information available. 
Theorem 4.10 (Aron, Boyd, Ryan, and Zalduendo, 2003 [15]). (a) Let $X$ be a real Banach space which does not admit a positive definite 4homogeneous polynomial. Then for every 2 -homogeneous polynomial $P$ on $X$, there is a nonseparable subspace of $X$ on which $P$ is identically zero.

(b) Let $X$ be a real Banach space of type 2. Then either $X$ admits a positive definite 2-homogeneous polynomial or every $P \in \mathcal{P}\left({ }^{2} X\right)$ has an nonseparable subspace on which it is identically zero.

It is worth noticing the connection with the geometry of the domain $X$. The requirement of type 2 in the above result is necessary for the use of the Extension Theorem of Maurey.

Still regarding results related to positive definite polynomials, for the case of a compact topological Hausdorff space $K$, the following dichotomy holds:

Theorem 4.11 (Férrer, 2007 [141]). The space $C(K)$ satisfies the following dichotomy. Either

(i) it admits a positive definite continuous 2-homogeneous real-valued polynomial, or

(ii) every continuous 2-homogeneous real-valued polynomial vanishes in a nonseparable closed linear subspace.

When $X=c_{0}(\Gamma)$ the following result holds for general polynomials (not necessarily homogeneous):

Theorem 4.12 (Férrer, 2007 [141]). Let $\Gamma$ be an uncountable set. If $P$ : $c_{0}(\Gamma) \rightarrow \mathbb{R}$ is a continuous polynomial, then there is a closed linear subspace $E$ of $c_{0}(\Gamma)$ such that $E \subset P^{-1}(0)$ and $E$ is isometric to $c_{0}(\Gamma)$.

(A3) For the last question we actually have a negative answer, as the following result shows.

Theorem 4.13 (Avilés and Todorcevic 2009, 28]). There exists a 2-homogeneous polynomial $P: \ell_{1}(\mathfrak{c}) \rightarrow \mathbb{C}$ such that $P^{-1}(0)$ contains both separable and nonseparable maximal subspaces.

Also [28] provides new perspectives on the investigation of zeros of complex polynomials, including new techniques and, especially, connections with results from [265] related to the existence of certain partitions with special properties.

As a final remark to this section, we would like to emphasize that this particular topic of study (subsets and subspaces of zeros of polynomials) has just recently started to develop. Thus, although it has rapidly caught the eye of many researchers in the field, there is still plenty of ongoing work on it. We believe that, in the years to come, this topic will certainly experience a large growth.

\section{Some remarks and conclusions. General techniques}

This last section will be devoted to providing several remarks on very particular results (not treated earlier) about lineability, operator theory, and the classical problem of the lineability of the set of norm-attaining functionals. Later, a number of heuristic comments will be given and, finally, some general techniques will be considered. 
Remark 5.1. Besides all the examples shown in the preceding sections, together with the different areas in which the topic of lineability has had its influence, the theory of absolutely - and nonabsolutely - summing operators has also attracted the attention of some authors when working on lineability. We refer the interested reader to [110, 112, 113, 201,242. Also, and more particularly, Pellegrino and Teixeira 236] provided some results within the framework of norm-attaining operators, results that were later generalized in 2011 by Botelho, Diniz, Fávaro, and Pellegrino [109.

Remark 5.2. Regarding a classical problem on norm-attaining functionals, a wellknown theorem by E. Bishop and R. Phelps (1961 [93]) states that for any Banach space $X$, the set $\mathcal{N} \mathcal{A}(X):=\left\{\varphi \in X^{*}\right.$ : attains the norm $\}$ is a dense subset of $X^{*}$. It is natural to ask about the lineability of the set $\mathcal{N} \mathcal{A}(X)$. For many specific spaces $X$, the answer is routine. For example,

$$
\mathcal{N} \mathcal{A}\left(c_{0}\right)=\left\{x=\left(x_{n}\right) \in \ell_{1}: x_{n}=0 \text { for all sufficiently large } n\right\} .
$$

In this case, $\mathcal{N} \mathcal{A}\left(c_{0}\right)$ is itself a dense vector subspace of $c_{0}^{*}=\ell_{1}$. However, note that $\mathcal{N} \mathcal{A}\left(c_{0}\right)$ contains no infinite dimensional closed subspace, so here we have lineability without spaceability. On the other hand,

$$
\mathcal{N} \mathcal{A}\left(\ell_{1}\right)=\left\{y=\left(y_{n}\right) \in \ell_{\infty}:\|y\|_{\infty}=\max _{n}\left|y_{n}\right|\right\}
$$

is not a subspace. Indeed, both $y=(-1,0,0, \ldots)$ and $y^{\prime}=\left(1, \frac{1}{2}, \frac{2}{3}, \ldots, \frac{n}{n+1}, \ldots\right)$ are in $\mathcal{N} \mathcal{A}\left(\ell_{1}\right)$ although $y+y^{\prime} \notin \mathcal{N} \mathcal{A}\left(\ell_{1}\right)$. Furthermore, $\mathcal{N} \mathcal{A}\left(\ell_{1}\right)$ contains both dense vector subspaces and infinite dimensional closed subspaces. This raises two questions:

When is $\mathcal{N} \mathcal{A}(X)$ a vector space? When is $\mathcal{N} \mathcal{A}(X)$ spaceable?

These questions have been deeply studied in [35] by Bandyopadhyay and Godefroy, who provided, among other results, conditions that ensure that $\mathcal{N} \mathcal{A}(X)$ is not spaceable; see also the recent works [1,153 for more results on the linear structure of $\mathcal{N} \mathcal{A}(X)$. Very recently, García-Pacheco and Puglisi [158] showed that every Banach space admitting an infinite dimensional separable quotient can be equivalently renormed in such a way that the set of its norm attaining functionals contains an infinite dimensional linear subspace. Nevertheless, the general problem of the 2-lineability of the set of norm-attaining functional in any Banach space is still open.

After the previous remarks, let us raise some considerations related to the Baire category theorem and lineability. Let $(X, d)$ be a complete metric space. A subset $E \subset X$ is said to be porous if there exist a constant $a \in(0,1)$ and a radius $r_{0}>0$ such that for every $x \in X$ and every $r \in\left(0, r_{0}\right)$ there is $y \in X$ with $B(y, a r) \subset B(x, r) \backslash E$. The set $E$ is said to be $\sigma$-porous if $E$ can be written as a countable union of porous subsets of $X$. If $E$ is porous, then it is nowhere dense. Hence $\sigma$-porous sets are of first category. The converse is false. Thus, it is in general a stronger statement to say that a set has $\sigma$-porous complement than to say that it is residual. There is some recent work on this topic. For instance, J. Borwein and X. Wang [106] show that if $X$ is a separable Banach space, then the set of all nonexpansive Lipschitz functions with maximal Clarke subdifferentials is not only residual but has $\sigma$-porous complement. In [106] the authors do not mention lineability, but our guess is that this property is lineable. Let us point out that, just recently, more examples of cases relating porosity and strong algebrability (in the case of continuous functions on locally compact groups) were provided in [5]. 
So far, we have seen many cases in which a set of functions having an exotic property is residual, so that its complement is of first category. We wonder in how many cases it is true that the complement is $\sigma$-porous. For instance, Bayart [46] demonstrated that the set $H C\left(C_{\varphi}\right)$ of hypercyclic functions with respect to the composition operator $C_{\varphi}$ generated by a nonelliptic automorphism $\varphi$ (recall that this set is residual, dense-lineable and spaceable, see Section 3 ) has no $\sigma$-porous complement. Also, and as we saw in Theorem 2.8, although being a residual set is "symptomatic" of containing large subspaces, there is clearly no immediate implication: recall, for instance, the example of the set $\mathcal{U}\left(\left(\Delta_{\alpha_{n}}\right)\right)$ given in Subsection 3.2.1. Also, in 33, there is another example in this direction: for any $x \in \ell_{1}$ define $E(x)=\left\{a \in \mathbb{R}: \exists A \subset \mathbb{N}\right.$ such that $\left.\sum_{n \in A} x(n)=a\right\}$, and let $\mathcal{C}$ be the set of all $x \in \ell_{1}$ for which $E(x)$ is homeomorphic to the Cantor set. Then $\mathcal{C}$ is residual, strongly $\mathfrak{c}$-algebrable, but not spaceable.

Certainly, and after what we have been seeing throughout this paper, one could think that almost everything is lineable, dense-lineable, or spaceable. This is certainly not the case, as we have already seen in, for instance, Theorems 2.8 and 2.17. In spite of this, and in a much more general frame, the following has been established.

Theorem 5.3 (Aron, García-Pacheco, Pérez-García, and Seoane-Sepúlveda, 2009 20]).

(a) Let $X$ be an infinite dimensional Banach space. There exists a subset $M \subset$ $X$ such that $M$ is spaceable and dense, although it is not dense-lineable.

(b) Let $X$ be an infinite dimensional Banach space. There exists a subset $M \subset$ $X$ which is lineable and dense, but which is not spaceable. If $X$ is separable, then $M$ can also be chosen to be dense-lineable.

One conclusion that we can infer from what we have been presenting in this survey is that a very common (and possibly the only!) constructive strategy to obtain lineability is the following: once a mathematical object enjoying a particular property is found, one tries to modify it in order to construct a basis for the potential candidate to linear space. Thus, almost all techniques are, at this time, constructive. Only a handful of existence techniques (although not very general ones) have been obtained in recent years. Here, and to conclude this survey, we will give a brief account of them. It was not until very recently that some existence results to guarantee the lineability of certain sets were given. These mentioned results, although not giving the specific linear space, supply the positive answer we are looking for in this area. Due to the lack of existence results in this topic, we believe that it is interesting to have this kind of machine-proving result that, right away, guarantees the lineability of a given family of functions. In order to do that, we relate the notion of lineability with that of additivity, introduced by T. Natkaniec in [232, 233] and thoroughly studied by F. E. Jordan in his Ph.D. dissertation [193].

Definition 5.4. Let $\mathcal{F} \subset \mathbb{R}^{\mathbb{R}}$. The additivity of $\mathcal{F}$ is defined as the following cardinal number:

$$
\mathcal{A}(\mathcal{F})=\min \left(\left\{\operatorname{card} F: F \subset \mathbb{R}^{\mathbb{R}}, \varphi+F \not \subset \mathcal{F}, \forall \varphi \in \mathbb{R}^{\mathbb{R}}\right\} \cup\left\{\left(2^{\mathfrak{c}}\right)^{+}\right\}\right),
$$

where $\left(2^{\mathfrak{c}}\right)^{+}$stands for the successor cardinal of $2^{\mathfrak{c}}$.

The above definition gives us, roughly, the biggest cardinal number $\kappa$ for which every family $\mathcal{G}$, with card $\mathcal{G}<\kappa$, can be translated into $\mathcal{F}$. Let us remark that for 
some families the additivity is relatively "easy" to compute, whereas lineability is, in general, "hard" to calculate.

Additivity and lineability have not been related until very recently. Although it may seem like this concept has nothing to do with the concept of lineability, it actually has a lot to do with it:

Theorem 5.5 (Gámez-Merino, Muñoz, Seoane-Sepúlveda, $2010[150]$ ). Let $\mathcal{F} \subset \mathbb{R}^{\mathbb{R}}$ be star-like, that is, $\alpha \mathcal{F} \subset \mathcal{F}$ for all $\alpha \in \mathbb{R}$. If $\mathfrak{c} \leq \mathcal{A}(\mathcal{F}) \leq 2^{\mathfrak{c}}$, then $\mathcal{F}$ is $\mathcal{A}(\mathcal{F})$ lineable.

Theorem 5.5 can be applied to many classes of functions, such as those presented in Definition 2.15. The result provides a technique that can only be used or applied within the framework of $\mathbb{R}^{\mathbb{R}}$. It would be very interesting if a dual result of Theorem 5.5 above could also be found for algebrability; that is, is there any cardinal invariant that relates to the concept of algebrability as the additivity does with lineability?

On the other hand, a few criteria have appeared recently that tell us how to get dense-lineability from the mere lineability. The idea that is in the core of these results is to obtain the desired dense subspace by adding small vectors coming from a known lineable set to the vectors of a dense subset. Such criteria are contained in the next two theorems. According to [20], if $A$ and $B$ are subsets of a vector space $X$, then $A$ is said to be stronger than $B$ provided that $A+B \subset A$.

Theorem 5.6 (Aron, García, Pérez, and Seoane-Sepúlveda 2009, [20]). Assume that $X$ is a metrizable separable topological vector space. If $A$ and $B$ are subsets of $X$ such that $A$ is lineable, $B$ is dense-lineable, and $A$ is stronger than $B$, then $A$ is dense-lineable.

Theorem 5.7 (Bernal, 2008, 2010 [67,69]). Assume that $X$ is a metrizable separable topological vector space. Suppose that $\Gamma$ is a family of vector subspaces of $X$ such that $\bigcap_{S \in \Gamma} S$ is dense in $X$. We have:

(a) If $\mu$ is an infinite cardinal number such that $\bigcap_{S \in \Gamma}(X \backslash S)$ is $\mu$-lineable, then it contains a dense vector subspace of dimension $\mu$.

(b) In particular, if $\bigcap_{S \in \Gamma}(X \backslash S)$ is lineable, then it is dense-lineable. And if $\bigcap_{S \in \Gamma}(X \backslash S)$ is maximal-lineable then it is maximal-dense-lineable.

Some of the lineability results mentioned in the present paper-concerning nowhere differentiable functions, nowhere analytic functions, dense hypercyclic manifolds, strict-order integrable functions, nowhere monotone functions, functions with everywhere unbounded derivatives, and strongly annular functions, among others, and which are contained in [20,67,69,71] — can be deduced by using Theorems 5.6 or 5.7

Concerning closed subspaces, it should be said that there are not many explicit spaceability general criteria in the related literature. A nice one in a rather abstract context is due to Wilansky and Kalton (see [201,272]): if $\mathrm{X}$ is a Fréchet space and $Y \subset X$ is a closed linear subspace, then the complement $X \backslash Y$ is spaceable if and only if $Y$ has infinite codimension. Kitson and Timoney exploited it to obtain the following theorem.

Theorem 5.8 (Kitson and Timoney, 2011 [201]). Let $Z_{n}(n \in \mathbb{N})$ be Banach spaces and $X$ a Fréchet space. Let $T_{n}: Z_{n} \rightarrow X$ be continuous linear mappings and $Y$ the linear span of $\bigcup_{n} T_{n}\left(Z_{n}\right)$. If $Y$ is not closed in $X$, then the complement $X \backslash Y$ is spaceable. 
This result can be used, for instance, to prove spaceability of the nonabsolutely convergent power series in the disc algebra $A(\mathbb{D})$ and of the nonabsolutely $p$-summing operators between certain pairs of Banach spaces. Also, some assertions in Subsection 2.4.2 may be inferred of Theorem 5.8. As the authors of [201] suggest, it would be interesting to know if this theorem can be extended to allow $Z_{n}$ to be a Fréchet space. In the more restrictive setting of function spaces, we have the following statement. Recall that the support of a function $f: \Omega \rightarrow \mathbb{K}$ is the set $\sigma(f)=\{x \in \Omega: f(x) \neq 0\}$.

Theorem 5.9 (Bernal-González and Ordóñez-Cobrera, 2012 [84]). Let $\Omega$ be a nonempty set. Assume that $(X,\|\cdot\|)$ is a Banach space of $\mathbb{K}$-valued functions on $\Omega$ and that $S$ is a nonempty subset of $X$ satisfying the following properties:

(i) If $\left(g_{n}\right) \subset X$ satisfies $g_{n} \rightarrow g$ in $X$, then there is a subsequence $\left(n_{k}\right) \subset \mathbb{N}$ such that, for every $x \in \Omega, g_{n_{k}}(x) \rightarrow g(x)$.

(ii) There is a constant $C \in(0,+\infty)$ such that $\|f+g\| \geq C\|f\|$ for all $f, g \in X$ with $\sigma(f) \cap \sigma(g)=\varnothing$.

(iii) $\alpha f \in S$ for all $\alpha \in \mathbb{K}$ and all $f \in S$.

(iv) If $f, g \in X$ are such that $f+g \in S$ and $\sigma(f) \cap \sigma(g)=\varnothing$, then $f, g \in S$.

(v) There is a sequence of functions $f_{n}(n \in \mathbb{N})$ with pairwise disjoint supports such that, for all $n \in \mathbb{N}, f_{n} \in X \backslash S$.

Then $X \backslash S$ is spaceable.

With this statement in hand, one can derive, for instance, some assertions about Lebesgue spaces; see Subsection 2.4.2. Theorem [5.9 also applies (see 84]) to the space $X=C B V[0,1]$ of bounded variation continuous functions on $[0,1]$, endowed with the norm $\|f\|=\sup _{[0,1]}+\operatorname{Variation}_{[0,1]} f$, yielding that the set $\{f \in$ $C B V[0,1]: f$ is not absolutely continuous in $[0,1]\}$ is spaceable.

Just recently, a new technique has been developed that can be applied to the framework of sequence spaces; see [107. The authors actually obtain maximalspaceability by providing a series of conditions that, once fulfilled, guarantee the existence of a closed infinite dimensional subspace inside sets of certain topological vector sequence spaces. Their general result [107, Theorem 3.1], which is obtained by making use of the notion of "sequence functor" (see [107, Definition 2.1]), applies to a large amount of cases, most of them never studied before, such as the sets $c_{0}(X) \backslash \bigcup_{p>0} \ell_{p}^{w}(X), \ell_{p}(X) \backslash \bigcup_{0<q<p} \ell_{q}^{w}(X)$, or $\ell_{p}^{u}(X) \backslash \bigcup_{0<q<p} \ell_{q}^{w}(X)$ (among many others), where $X$ is any infinite dimensional Banach space, $\ell_{p}^{w}(X)$ denotes the Banach ( $p$-Banach if $0<p<\infty)$ space of all $X$-valued weakly $p$-summable sequences, and $\ell_{p}^{u}(X)$ stands for the closed subspace of $\ell_{p}^{w}(X)$ formed by all unconditionally $p$-summable sequences; see [107, Corollaries 3.2-3.7].

There is no need in saying that due to the complexity of many of these lineability problems, a Baire category theorem-type result - or any general existence technique for that matter - would be extremely valuable in this recently coined theory.

\section{ABOUT THE AUTHORS}

Luis Bernal-González graduated in 1980 from the Universidad de Sevilla (Spain) where he earned his Ph.D. in 1984. He is currently a professor at the same university. He was an invited speaker at the International Congress on Hypercyclicity and Chaos for Linear Operators and Semigroups in Valencia (Spain) in 2009. His main interests are complex analysis and operator theory. 
Daniel Pellegrino is a professor in the Department of Mathematics at the Universidade Federal da Paraíba (Brazil). He earned his Ph.D. at the Universidade Estadual de Campinas (Brazil) in 2002. He is an affiliate member of the Brazilian Academy of Sciences and a CNPq researcher.

Juan B. Seoane-Sepúlveda received his Ph.D. at the Universidad de Cádiz (Spain) jointly with Universität Karlsruhe (Germany) in 2005. His second Ph.D. was earned at Kent State University (Kent, Ohio, USA) in 2006. His main interests include real analysis, operator theory, and lineability and spaceability. He is currently a professor at Universidad Complutense de Madrid (Spain).

\section{ACKNOWLEDGMENTS}

The authors wish to thank Professor Richard M. Aron for his help in the elaboration of this survey and Professor Antonio Bonilla for some suggestions concerning Section 3. The authors express their gratitude to the anonymous referees, whose thorough analysis and insightful remarks improved the text.

\section{REFERENCES}

[1] M. D. Acosta, A. Aizpuru, R. M. Aron, and F. J. García-Pacheco, Functionals that do not attain their norm, Bull. Belg. Math. Soc. Simon Stevin 14 (2007), no. 3, 407-418. MR.2387038 (2009i:46021)

[2] A. Aizpuru, C. Pérez-Eslava, F. J. García-Pacheco, and J. B. Seoane-Sepúlveda, Lineability and coneability of discontinuous functions on $\mathbb{R}$, Publ. Math. Debrecen 72 (2008), no. 1-2, 129-139. MR2376864 (2008m:26002)

[3] A. Aizpuru, C. Pérez-Eslava, and J. B. Seoane-Sepúlveda, Linear structure of sets of divergent sequences and series, Linear Algebra Appl. 418 (2006), no. 2-3, 595-598, DOI 10.1016/j.laa.2006.02.041. MR.2260214 (2008h:40001)

[4] I. Akbarbaglu and S. Maghsoudi, Large structures in certain subsets of Orlicz spaces, Linear Algebra Appl. 438 (2013), no. 11, 4363-4373, DOI 10.1016/j.laa.2013.01.038. MR3034537

[5] I. Akbarbaglu, S. Maghsoudi, and J. B. Seoane-Sepúlveda, Porous sets and lineability of continuous functions on locally compact groups, J. Math. Anal. Appl. 406 (2013), no. 1, $211-218$.

[6] A. Albanese, X. Barrachina, E. M. Mangino, and A. Peris, Distributional chaos for strongly continuous semigroups of operators, Comm. Pure Appl. Analysis 12 (2013), no. 5, 20692082.

[7] P. C. Allaart and K. Kawamura, The improper infinite derivatives of Takagi's nowhere-differentiable function, J. Math. Anal. Appl. 372 (2010), no. 2, 656-665, DOI 10.1016/j.jmaa.2010.06.059. MR2678891 (2011k:26004)

[8] S. I. Ansari, Existence of hypercyclic operators on topological vector spaces, J. Funct. Anal. 148 (1997), no. 2, 384-390, DOI 10.1006/jfan.1996.3093. MR.1469346 (98h:47028a)

[9] D. H. Armitage, A non-constant continuous function on the plane whose integral on every line is zero, Amer. Math. Monthly 101 (1994), no. 9, 892-894, DOI 10.2307/2975138. MR 1300495 (95m:30053)

[10] D. H. Armitage, Dense vector spaces of universal harmonic functions, Advances in multivariate approximation (Witten-Bommerholz, 1998), Math. Res., vol. 107, Wiley-VCH, Berlin, 1999, pp. 33-42. MR1797219 (2001k:31004)

[11] D. H. Armitage, Entire functions decaying rapidly on strips, Quaest. Math. 23 (2000), no. 4, 417-424, DOI 10.2989/16073600009485988. MR.1810291 (2001i:30024)

[12] R. Aron, An introduction to polynomials on Banach spaces, Extracta Math. 17 (2002), no. 3, 303-329. IV Course on Banach Spaces and Operators (Spanish) (Laredo, 2001). MR.1995411 (2004f:46053)

[13] R. M. Aron, Linearity in non-linear situations, Advanced courses of mathematical analysis. II, World Sci. Publ., Hackensack, NJ, 2007, pp. 1-15, DOI 10.1142/9789812708441_0001. MR2334322(2008f:46001) 
[14] R. Aron, J. Bès, F. León, and A. Peris, Operators with common hypercyclic subspaces, J. Operator Theory 54 (2005), no. 2, 251-260. MR2186352(2006h:47010)

[15] R. M. Aron, C. Boyd, R. A. Ryan, and I. Zalduendo, Zeros of polynomials on Banach spaces: the real story, Positivity 7 (2003), no. 4, 285-295, DOI 10.1023/A:1026278115574. MR.2017308(2004j:46066)

[16] R. M. Aron, J. A. Conejero, A. Peris, and J. B. Seoane-Sepúlveda, Sums and products of bad functions, Function spaces, Contemp. Math., vol. 435, Amer. Math. Soc., Providence, RI, 2007, pp. 47-52, DOI 10.1090/conm/435/08365. MR2359417 (2008m:46062)

[17] R. M. Aron, J. A. Conejero, A. Peris, and J. B. Seoane-Sepúlveda, Powers of hypercyclic functions for some classical hypercyclic operators, Integral Equations Operator Theory $\mathbf{5 8}$ (2007), no. 4, 591-596, DOI 10.1007/s00020-007-1490-4. MR2329137(2008e:47017)

[18] R. M. Aron, J. A. Conejero, A. Peris, and J. B. Seoane-Sepúlveda, Uncountably generated algebras of everywhere surjective functions, Bull. Belg. Math. Soc. Simon Stevin 17 (2010), no. 3, 571-575. MR2731374 (2011g:46041)

[19] R. Aron, D. García, and M. Maestre, Linearity in non-linear problems, RACSAM. Rev. R. Acad. Cienc. Exactas Fís. Nat. Ser. A Mat. 95 (2001), no. 1, 7-12 (English, with English and Spanish summaries). MR1899348(2003b:46062)

[20] R. M. Aron, F. J. García-Pacheco, D. Pérez-García, and J. B. Seoane-Sepúlveda, On dense-lineability of sets of functions on $\mathbb{R}$, Topology 48 (2009), no. 2-4, 149-156, DOI 10.1016/j.top.2009.11.013. MR.2596209 (2011c:26011)

[21] R. Aron, R. Gonzalo, and A. Zagorodnyuk, Zeros of real polynomials, Linear and Multilinear Algebra 48 (2000), no. 2, 107-115, DOI 10.1080/03081080008818662. MR.1813438 (2001k:12002)

[22] R. Aron, V. I. Gurariy, and J. B. Seoane-Sepúlveda, Lineability and spaceability of sets of functions on $\mathbb{R}$, Proc. Amer. Math. Soc. 133 (2005), no. 3, 795-803 (electronic), DOI 10.1090/S0002-9939-04-07533-1. MR2113929(2006i:26004)

[23] R. M. Aron and P. Hájek, Odd degree polynomials on real Banach spaces, Positivity 11 (2007), no. 1, 143-153, DOI 10.1007/s11117-006-2035-9. MR2297328(2007m:46064)

[24] R. M. Aron and P. Hájek, Zero sets of polynomials in several variables, Arch. Math. (Basel) 86 (2006), no. 6, 561-568, DOI 10.1007/s00013-006-1314-9. MR2241604 (2008c:46063)

[25] R. M. Aron, D. Pérez-García, and J. B. Seoane-Sepúlveda, Algebrability of the set of nonconvergent Fourier series, Studia Math. 175 (2006), no. 1, 83-90, DOI 10.4064/sm175-1-5. MR 2261701 (2007k:42007)

[26] R. M. Aron and M. P. Rueda, A problem concerning zero-subspaces of homogeneous polynomials, Linear Topol. Spaces Complex Anal. 3 (1997), 20-23. Dedicated to Professor Vyacheslav Pavlovich Zahariuta. MR1632480 (99e:13032)

[27] R. M. Aron and J. B. Seoane-Sepúlveda, Algebrability of the set of everywhere surjective functions on $\mathbb{C}$, Bull. Belg. Math. Soc. Simon Stevin 14 (2007), no. 1, 25-31. MR2327324 (2008d:26016)

[28] A. Avilés and S. Todorcevic, Zero subspaces of polynomials on $l_{1}(\Gamma)$, J. Math. Anal. Appl. 350 (2009), no. 2, 427-435, DOI 10.1016/j.jmaa.2007.08.020. MR2474778 (2009k:46077)

[29] D. Azagra, G. A. Muñoz-Fernández, V. M. Sánchez, and J. B. Seoane-Sepúlveda, Riemann integrability and Lebesgue measurability of the composite function, J. Math. Anal. Appl. 354 (2009), no. 1, 229-233, DOI 10.1016/j.jmaa.2008.12.033. MR2510434 (2010i:26016)

[30] A. G. Bacharoglu, Universal Taylor series on doubly connected domains, Results in Maths. 53 (2009), no. 1-2, 9-18.

[31] M. Balcerzak, A. Bartoszewicz, and M. Filipczac, Nonseparable spaceability and strong algebrability of sets of continuous singular functions, J. Math. Anal. Appl. (to appear).

[32] M. Balcerzak, K. Ciesielski, and T. Natkaniec, Sierpinski-Zygmund functions that are Darboux, almost continuous, or have a perfect road, Arch. Math. Logic 37 (1997), no. 1, 29-35, DOI 10.1007/s001530050080. MR1485861 (98k:26005)

[33] T. Banakh, A. Bartoszewicz, S. Głąb, and E. Szymonik, Algebrability, lineability and the subsums of series, Colloq. Math. 129 (2012), no. 3, 75-85.

[34] T. Banakh, A. Plichko, and A. Zagorodnyuk, Zeros of quadratic functionals on non-separable spaces, Colloq. Math. 100 (2004), no. 1, 141-147, DOI 10.4064/cm100-1-13. MR2079354 (2005g:46047) 
[35] P. Bandyopadhyay and G. Godefroy, Linear structures in the set of norm-attaining functionals on a Banach space, J. Convex Anal. 13 (2006), no. 3-4, 489-497. MR.2291549 (2007k:46020)

[36] C. S. Barroso, G. Botelho, V. V. Fávaro, and D. Pellegrino, Lineability and spaceability for the weak form of Peano's theorem and vector-valued sequence spaces, Proc. Amer. Math. Soc. 141 (2013), no. 6, 1913-1923, DOI 10.1090/S0002-9939-2012-11466-2. MR3034418

[37] A. Bartoszewicz, M. Bienias, and S. Głąb, Independent Bernstein sets and algebraic constructions, J. Math. Anal. Appl. 393 (2012), no. 1, 138-143, DOI 10.1016/j.jmaa.2012.03.007. MR2921655

[38] A. Bartoszewicz and S. Głąb, Algebrability of conditionally convergent series with Cauchy product, J. Math. Anal. Appl. 385 (2012), no. 2, 693-697, DOI 10.1016/j.jmaa.2011.07.008. MR.2834845 (2012g:40001)

[39] A. Bartoszewicz and S. Głąb, Strong algebrability of sets of sequences and functions, Proc. Amer. Math. Soc. 141 (2013), no. 3, 827-835, DOI 10.1090/S0002-9939-2012-11377-2. MR.3003676

[40] A. Bartoszewicz, S. Głąb, D. Pellegrino, and J. B. Seoane-Sepúlveda, Algebrability, nonlinear properties, and special functions, Proc. Amer. Math. Soc., in press.

[41] A. Bartoszewicz, S. Głąb, and T. Poreda, On algebrability of nonabsolutely convergent series, Linear Algebra Appl. 435 (2011), no. 5, 1025-1028, DOI 10.1016/j.laa.2011.02.008. MR2807216 (2012e:40001)

[42] F. Bastin, C. Esser, and S. Nicolay, Prevalence of "nowhere analyticity", Studia Math. 210 (2012), no. 3, 239-246, DOI 10.4064/sm210-3-4. MR2983462

[43] F. Bayart, Linearity of sets of strange functions, Michigan Math. J. 53 (2005), no. 2, 291303, DOI 10.1307/mmj/1123090769. MR2152701(2006b:46048)

[44] F. Bayart, Topological and algebraic genericity of divergence and universality, Studia Math. 167 (2005), no. 2, 161-181, DOI 10.4064/sm167-2-4. MR2134382(2006b:46024)

[45] F. Bayart, Common hypercyclic subspaces, Integral Equations Operator Theory 53 (2005), no. 4, 467-476, DOI 10.1007/s00020-004-1316-6. MR2187432 (2006k:47016)

[46] F. Bayart, Porosity and hypercyclic operators, Proc. Amer. Math. Soc. 133 (2005), no. 11, 3309-3316 (electronic), DOI 10.1090/S0002-9939-05-07842-1. MR2161154 (2006f:47008)

[47] F. Bayart and S. Grivaux, Frequently hypercyclic operators, Trans. Amer. Math. Soc. 358 (2006), no. 11, 5083-5117 (electronic), DOI 10.1090/S0002-9947-06-04019-0. MR2231886 (2007e:47013)

[48] F. Bayart and S. Grivaux, Invariant Gaussian measures for operators on Banach spaces and linear dynamics, Proc. Lond. Math. Soc. (3) 94 (2007), no. 1, 181-210, DOI 10.1112/plms/pdl013. MR2294994(2008i:47019)

[49] F. Bayart, K.-G. Grosse-Erdmann, V. Nestoridis, and C. Papadimitropoulos, Abstract theory of universal series and applications, Proc. Lond. Math. Soc. (3) 96 (2008), no. 2, 417-463, DOI 10.1112/plms/pdm043. MR2396846(2009j:30006)

[50] F. Bayart, S. V. Konyagin, and H. Queffélec, Convergence almost everywhere and divergence everywhere of Taylor and Dirichlet series, Real Anal. Exchange 29 (2003/04), no. 2, 557586. MR2083797(2005e:42026)

[51] F. Bayart and É. Matheron, Hypercyclic operators failing the hypercyclicity criterion on classical Banach spaces, J. Funct. Anal. 250 (2007), no. 2, 426-441, DOI 10.1016/j.jfa.2007.05.001. MR2352487 (2008k:47016)

[52] F. Bayart and É. Matheron, Dynamics of linear operators, Cambridge Tracts in Mathematics, vol. 179, Cambridge University Press, Cambridge, 2009. MR 2533318 (2010m:47001)

[53] F. Bayart and V. Nestoridis, Universal Taylor series have a strong form of universality, J. Anal. Math. 104 (2008), 69-82, DOI 10.1007/s11854-008-0017-5. MR2403430(2009e:30003)

[54] F. Bayart and L. Quarta, Algebras in sets of queer functions, Israel J. Math. 158 (2007), 285-296, DOI 10.1007/s11856-007-0014-x. MR2342549 (2008g:26006)

[55] B. Beauzamy, Un opérateur, sur l'espace de Hilbert, dont tous les polynômes sont hypercycliques, C. R. Acad. Sci. Paris Sér. I Math. 303 (1986), no. 18, 923-925 (French, with English summary). MR 873395 (88g:47010)

[56] B. Beauzamy, Introduction to operator theory and invariant subspaces, North-Holland Mathematical Library, vol. 42, North-Holland Publishing Co., Amsterdam, 1988. MR967989 (90d:47001) 
[57] T. Bermúdez, A. Bonilla, F. Martínez-Giménez, and A. Peris, Li-Yorke and distributionally chaotic operators, J. Math. Anal. Appl. 373 (2011), no. 1, 83-93, DOI 10.1016/j.jmaa.2010.06.011. MR2684459(2011j:47031)

[58] L. Bernal-González, A lot of “counterexamples" to Liouville's theorem, J. Math. Anal. Appl. 201 (1996), no. 3, 1002-1009, DOI 10.1006/jmaa.1996.0298. MR.1400577 (97d:30036)

[59] L. Bernal-González, Small entire functions with extremely fast growth, J. Math. Anal. Appl. 207 (1997), no. 2, 541-548, DOI 10.1006/jmaa.1997.5312. MR.1438930 (98b:30028)

[60] L. Bernal-González, On hypercyclic operators on Banach spaces, Proc. Amer. Math. Soc. 127 (1999), no. 4, 1003-1010, DOI 10.1090/S0002-9939-99-04657-2. MR1476119 (99f:47010)

[61] L. Bernal-González, Densely hereditarily hypercyclic sequences and large hypercyclic manifolds, Proc. Amer. Math. Soc. 127 (1999), no. 11, 3279-3285, DOI 10.1090/S0002-9939-9905185-0. MR 1646318 (2000b:47047)

[62] L. Bernal-González, Hypercyclic sequences of differential and antidifferential operators, J. Approx. Theory 96 (1999), no. 2, 323-337, DOI 10.1006/jath.1998.3237. MR.1671201 (2000b:47081)

[63] L. Bernal-González, Universal images of universal elements, Studia Math. 138 (2000), no. 3, 241-250. MR1758857 (2001f:47002)

[64] L. Bernal-González, Linear Kierst-Szpilrajn theorems, Studia Math. 166 (2005), no. 1, 5569, DOI 10.4064/sm166-1-4. MR2108318 (2006m:30100)

[65] L. Bernal-González, Hypercyclic subspaces in Fréchet spaces, Proc. Amer. Math. Soc. 134 (2006), no. 7, 1955-1961, DOI 10.1090/S0002-9939-05-08242-0. MR2215764 (2007a:47008)

[66] L. Bernal-González, Linear structure of weighted holomorphic non-extendibility, Bull. Austral. Math. Soc. 73 (2006), no. 3, 335-344, DOI 10.1017/S0004972700035371. MR2230644 (2007b:30068)

[67] L. Bernal-González, Dense-lineability in spaces of continuous functions, Proc. Amer. Math. Soc. 136 (2008), no. 9, 3163-3169, DOI 10.1090/S0002-9939-08-09495-1. MR2407080 (2009c:46038)

[68] L. Bernal-González, Lineability of sets of nowhere analytic functions, J. Math. Anal. Appl. 340 (2008), no. 2, 1284-1295, DOI 10.1016/j.jmaa.2007.09.048. MR2390929(2009d:46047)

[69] L. Bernal-González, Algebraic genericity of strict-order integrability, Studia Math. 199 (2010), no. 3, 279-293, DOI 10.4064/sm199-3-5. MR2678906 (2011g:46049)

[70] L. Bernal-González, Lineability of universal divergence of Fourier series, Integral Equations Operator Theory 74 (2012), no. 2, 271-279, DOI 10.1007/s00020-012-1984-6. MR2983066

[71] L. Bernal-González and A. Bonilla, Families of strongly annular functions: linear structure, Rev. Mat. Complut. 26 (2013), no. 1, 283-297, DOI 10.1007/s13163-011-0080-9. MR3016629

[72] L. Bernal-González, A. Bonilla, M. C. Calderón-Moreno, and J. A. Prado-Bassas, Maximal cluster sets of L-analytic functions along arbitrary curves, Constr. Approx. 25 (2007), no. 2, 211-219, DOI 10.1007/s00365-006-0636-5. MR2283498 (2007k:30055)

[73] L. Bernal-González, A. Bonilla, M. C. Calderón-Moreno, and J. A. Prado-Bassas, Universal Taylor series with maximal cluster sets, Rev. Mat. Iberoam. 25 (2009), no. 2, 757-780, DOI 10.4171/RMI/582. MR2569553(2011a:30004)

[74] L. Bernal-González and M. D. C. Calderón-Moreno, Dense linear manifolds of monsters, J. Approx. Theory 119 (2002), no. 2, 156-180, DOI 10.1006/jath.2002.3712. MR.1939280 (2003h:30042)

[75] L. Bernal-González, M. C. Calderón-Moreno, and W. Luh, Large linear manifolds of noncontinuable boundary-regular holomorphic functions, J. Math. Anal. Appl. 341 (2008), no. 1, 337-345, DOI 10.1016/j.jmaa.2007.10.014. MR2394088 (2009b:30068)

[76] L. Bernal-González, M. C. Calderón-Moreno, and W. Luh, Dense-lineability of sets of Birkhoff-universal functions with rapid decay, J. Math. Anal. Appl. 363 (2010), no. 1, 327335, DOI 10.1016/j.jmaa.2009.08.049. MR2559068(2010k:30029)

[77] L. Bernal-González, M. C. Calderón-Moreno, and J. A. Prado-Bassas, Maximal cluster sets along arbitrary curves, J. Approx. Theory 129 (2004), no. 2, 207-216, DOI 10.1016/j.jat.2004.06.003. MR2078649(2005f:30067)

[78] L. Bernal-González, M. C. Calderón-Moreno, and J. A. Prado-Bassas, Cyclicity of coefficient multipliers: linear structure, Acta Math. Hungar. 114 (2007), no. 4, 287-300, DOI 10.1007/s10474-007-5125-7. MR2305820(2008d:47017) 
[79] L. Bernal-González, M. C. Calderón-Moreno, and J. A. Prado-Bassas, Holomorphic operators generating dense images, Integral Equations Operator Theory 60 (2008), no. 1, 1-11, DOI 10.1007/s00020-007-1547-4. MR2380312(2009e:47022)

[80] L. Bernal-González, M. C. Calderón-Moreno, and J. A. Prado-Bassas, Large subspaces of compositionally universal functions with maximal cluster sets, J. Approx. Theory 164 (2012), no. 2, 253-267, DOI 10.1016/j.jat.2011.10.005. MR2864645

[81] L. Bernal-González and K.-G. Grosse-Erdmann, The hypercyclicity criterion for sequences of operators, Studia Math. 157 (2003), no. 1, 17-32, DOI 10.4064/sm157-1-2. MR.1980114 (2003m:47013)

[82] L. Bernal González and A. Montes-Rodríguez, Universal functions for composition operators, Complex Variables Theory Appl. 27 (1995), no. 1, 47-56. MR1316270 (96a:30041)

[83] L. B. González and A. M. Rodríguez, Non-finite-dimensional closed vector spaces of universal functions for composition operators, J. Approx. Theory 82 (1995), no. 3, 375-391, DOI 10.1006/jath.1995.1086. MR.1348728 (96f:30034)

[84] L. Bernal-González and M. Ordóñez Cabrera, Spaceability of strict order integrability, J. Math. Anal. Appl. 385 (2012), no. 1, 303-309, DOI 10.1016/j.jmaa.2011.06.043. MR.2834257 (2012g:46025)

[85] L. Bernal-González and J. A. Prado-Tendero, U-operators, J. Aust. Math. Soc. 78 (2005), no. 1, 59-89, DOI 10.1017/S1446788700015561. MR2129489(2006c:30039)

[86] N. Bernardes, A. Bonilla, V. Müller, and A. Peris, Li-Yorke chaos in Linear Dynamics, Preprint (2012).

[87] J. P. Bès, Invariant manifolds of hypercyclic vectors for the real scalar case, Proc. Amer. Math. Soc. 127 (1999), no. 6, 1801-1804, DOI 10.1090/S0002-9939-99-04720-6. MR 1485460 (99i:47002)

[88] J. P. Bès, Dynamics of composition operators with holomorphic symbol, Rev. Real Acad. Cien. Ser. A Mat., in press.

[89] J. Bès and J. A. Conejero, Hypercyclic subspaces in omega, J. Math. Anal. Appl. 316 (2006), no. 1, 16-23, DOI 10.1016/j.jmaa.2005.04.083. MR2201746 (2007b:47016)

[90] J. Bès and A. Peris, Hereditarily hypercyclic operators, J. Funct. Anal. 167 (1999), no. 1, 94-112, DOI 10.1006/jfan.1999.3437. MR.1710637 (2000f:47012)

[91] C. Bessaga and A. Pełczyński, On bases and unconditional convergence of series in Banach spaces, Studia Math. 17 (1958), 151-164. MR0115069 (22 \#5872)

[92] G. D. Birkhoff, Démonstration d'un théorème élémentaire sur les fonctions entières, C. R. Acad. Sci. Paris 189 (1929), 473-475.

[93] E. Bishop and R. R. Phelps, A proof that every Banach space is subreflexive, Bull. Amer. Math. Soc. 67 (1961), 97-98. MR0123174 (23 \#A503)

[94] H. Blumberg, New properties of all real functions, Trans. Amer. Math. Soc. 82 (1922), 53-61.

[95] P. du Bois-Reymond, Über den Gültigkeitsbereich der Taylorschen Reihenentwicklung, Math. Ann. 21 (1876), 109-117.

[96] D. D. Bonar and F. W. Carroll, Annular functions form a residual set, J. Reine Angew. Math. 272 (1975), 23-24. MR0417428 (54 \#5478)

[97] J. Bonet, F. Martínez-Giménez, and A. Peris, Linear chaos on Fréchet spaces, Internat. J. Bifur. Chaos Appl. Sci. Engrg. 13 (2003), no. 7, 1649-1655, DOI 10.1142/S0218127403007497. Dynamical systems and functional equations (Murcia, 2000). MR 2015614 (2004i:47016)

[98] J. Bonet, F. Martínez-Giménez, and A. Peris, Universal and chaotic multipliers on spaces of operators, J. Math. Anal. Appl. 297 (2004), no. 2, 599-611, DOI 10.1016/j.jmaa.2004.03.073. Special issue dedicated to John Horváth. MR2088683 (2005g:47006)

[99] J. Bonet and A. Peris, Hypercyclic operators on non-normable Fréchet spaces, J. Funct. Anal. 159 (1998), no. 2, 587-595, DOI 10.1006/jfan.1998.3315. MR.1658096 (99k:47044)

[100] A. Bonilla, "Counterexamples" to the harmonic Liouville theorem and harmonic functions with zero nontangential limits, Colloq. Math. 83 (2000), no. 2, 155-160. MR1758311 (2001d:31003)

[101] A. Bonilla, Small entire functions with infinite growth index, J. Math. Anal. Appl. 267 (2002), no. 1, 400-404, DOI 10.1006/jmaa.2001.7767. MR1886838 (2003d:30031)

[102] A. Bonilla, Universal harmonic functions, Quaest. Math. 25 (2002), 527-530. 
[103] A. Bonilla and K.-G. Grosse-Erdmann, On a theorem of Godefroy and Shapiro, Integral Equations Operator Theory 56 (2006), no. 2, 151-162, DOI 10.1007/s00020-006-1423-7. MR 2264513 (2007h:47020)

[104] A. Bonilla and K.-G. Grosse-Erdmann, Frequently hypercyclic operators and vectors, Ergodic Theory Dynam. Systems 27 (2007), no. 2, 383-404, DOI 10.1017/S014338570600085X. MR.2308137 (2008c:47016)

[105] A. Bonilla and K.-G. Grosse-Erdmann, Frequently hypercyclic subspaces, Monatsh. Math. 168 (2012), no. 3-4, 305-320, DOI 10.1007/s00605-011-0369-2. MR.2993952

[106] J. M. Borwein and X. Wang, Lipschitz functions with maximal Clarke subdifferentials are staunch, Bull. Austral. Math. Soc. 72 (2005), no. 3, 491-496, DOI 10.1017/S0004972700035322. MR2199651(2007a:49024)

[107] G. Botelho, D. Cariello, V. V. Fávaro, and D. Pellegrino, Maximal spaceability in sequence spaces, Linear Algebra Appl. 437 (2012), no. 12, 2978-2985, DOI 10.1016/j.laa.2012.06.043. MR2966612

[108] G. Botelho, D. Cariello, V. V. Fávaro, D. Pellegrino, and J. B. Seoane-Sepúlveda, Distinguished subspaces of $L_{p}$ of maximal dimension, Studia Math. (to appear).

[109] G. Botelho, D. Diniz, V. V. Fávaro, and D. Pellegrino, Spaceability in Banach and quasi-Banach sequence spaces, Linear Algebra Appl. 434 (2011), no. 5, 1255-1260, DOI 10.1016/j.laa.2010.11.012. MR.2763584(2011m:46006)

[110] G. Botelho, D. Diniz, and D. Pellegrino, Lineability of the set of bounded linear nonabsolutely summing operators, J. Math. Anal. Appl. 357 (2009), no. 1, 171-175, DOI 10.1016/j.jmaa.2009.03.062. MR2526816(2010d:47025)

[111] G. Botelho, V. V. Fávaro, D. Pellegrino, and J. B. Seoane-Sepúlveda, $L_{p}[0,1] \backslash \bigcup_{q>p} L_{q}[0,1]$ is spaceable for every $p>0$, Linear Algebra Appl. 436 (2012), no. 9, 2963-2965, DOI 10.1016/j.laa.2011.12.028. MR.2900689

[112] G. Botelho, M. C. Matos, and D. Pellegrino, Lineability of summing sets of homogeneous polynomials, Linear Multilinear Algebra 58 (2010), no. 1-2, 61-74, DOI 10.1080/03081080802095446. MR2641522(2011b:46072)

[113] G. Botelho, D. Pellegrino, and P. Rueda, Cotype and absolutely summing linear operators, Math. Z. 267 (2011), no. 1-2, 1-7, DOI 10.1007/s00209-009-0591-y. MR2772238 (2012e:46022)

[114] P. S. Bourdon, Invariant manifolds of hypercyclic vectors, Proc. Amer. Math. Soc. 118 (1993), no. 3, 845-847, DOI 10.2307/2160131. MR1148021 (93i:47002)

[115] P. S. Bourdon and J. H. Shapiro, The role of the spectrum in the cyclic behavior of composition operators, Memoirs Amer. Math. Soc. 596, AMS, Providence, Rhode Island, 1997.

[116] J. Bourgain, On the distribution of Dirichlet sums, J. Anal. Math. 60 (1993), 21-32.

[117] A. M. Bruckner, Differentiation of real functions, Lecture Notes in Mathematics, vol. 659, Springer, Berlin, 1978. MR507448 (80h:26002)

[118] D. Burdick and F. D. Lesley, Some uniqueness theorems for analytic functions, Amer. Math. Monthly 82 (1975), 152-155. MR0357766 (50 \#10234)

[119] Z. Buczolich, Solution to the gradient problem of C. E. Weil, Rev. Mat. Iberoamericana 21 (2005), no. 3, 889-910, DOI 10.4171/RMI/439. MR2231014(2007c:26012)

[120] M. C. Calderón-Moreno, Universal functions with small derivatives and extremely fast growth, Analysis (Munich) 22 (2002), no. 1, 57-66. MR1899914 (2003c:30023)

[121] F. S. Cater, Differentiable, nowhere analytic functions, Amer. Math. Monthly 91 (1984), no. 10, 618-624, DOI 10.2307/2323363. MR769526 (86b:26034)

[122] S. Charpentier, On the closed subspaces of universal series in Banach spaces and Fréchet spaces, Studia Math. 198 (2010), no. 2, 121-145, DOI 10.4064/sm198-2-2. MR2640073 (2011f:30101)

[123] J. Chmielowski, Domains of holomorphy of type $A^{k}$, Proc. Roy. Irish Acad. Sect. A 80 (1980), no. 1, 97-101. MR.581672 (81m:32014)

[124] C. K. Chui and M. N. Parnes, Approximation by overconvergence of a power series, J. Math. Anal. Appl. 36 (1971), 693-696. MR0291472 (45 \#563)

[125] K. Ciesielski and T. Natkaniec, Algebraic properties of the class of Sierpinski-Zygmund functions, Topology Appl. 79 (1997), no. 1, 75-99, DOI 10.1016/S0166-8641(96)00128-9. MR 1462608 (99c:04003)

[126] K. Ciesielski and T. Natkaniec, On Sierpiński-Zygmund bijections and their inverses, Topology Proc. 22 (1997), no. Spring, 155-164. MR1657922 (99k:26001) 
[127] K. Ciesielski and J. Pawlikowski, The covering property axiom, CPA, Cambridge Tracts in Mathematics, vol. 164, Cambridge University Press, Cambridge, 2004. A combinatorial core of the iterated perfect set model. MR2176267 (2006f:03002)

[128] J. A. Conejero, P. Jiménez-Rodríguez, G. A. Muñoz-Fernández, and J. B. Seoane-Sepúlveda, When the Identity Theorem "seems" to fail, Amer. Math. Monthly (to appear).

[129] J. A. Conejero, V. Müller, and A. Peris, Hypercyclic behaviour of operators in a hypercyclic $C_{0}$-semigroup, J. Funct. Anal. 244 (2007), no. 1, 342-348, DOI 10.1016/j.jfa.2006.12.008. MR 2294487(2007m:47013)

[130] M. de la Rosa and C. Read, A hypercyclic operator whose direct sum $T \oplus T$ is not hypercyclic, J. Operator Theory 61 (2009), no. 2, 369-380. MR2501011(2010e:47023)

[131] R. Deville and É. Matheron, Infinite games, Banach space geometry and the eikonal equation, Proc. Lond. Math. Soc. (3) 95 (2007), no. 1, 49-68, DOI 10.1112/plms/pdm005. MR2329548 (2008f:91007)

[132] E. Diamantopoulos, Ch. Kariofillis, and Ch. Mouratides, Universal Laurent series in finitely connected domains, Arch. Math. (Basel) 91 (2008), no. 2, 145-154, DOI 10.1007/s00013008-2470-x. MR2430798 (2009m:30002)

[133] J. Diestel, Sequences and series in Banach spaces, Graduate Texts in Mathematics, vol. 92, Springer-Verlag, New York, 1984. MR737004 (85i:46020)

[134] S. Dineen, Complex analysis on infinite-dimensional spaces, Springer Monographs in Mathematics, Springer-Verlag London Ltd., London, 1999. MR1705327 (2001a:46043)

[135] V. Drobot and M. Morayne, Continuous functions with a dense set of proper local maxima, Amer. Math. Monthly 92 (1985), no. 3, 209-211, DOI 10.2307/2322877. MR786345 (86e:26003)

[136] A. Dvoretzky and C. A. Rogers, Absolute and unconditional convergence in normed linear spaces, Proc. Nat. Acad. Sci. U. S. A. 36 (1950), 192-197. MR0033975 (11,525a)

[137] P. H. Enflo, V. I. Gurariy, and J. B. Seoane-Sepúlveda, Some results and open questions on spaceability in function spaces, Trans. Amer. Math. Soc. (to appear).

[138] P. H. Enflo, V. I. Gurariy, and J. B. Seoane-Sepúlveda, On Montgomery's conjecture and the distribution of Dirichlet sums, Preprint (2012).

[139] M. Fabián, D. Preiss, J. H. M. Whitfield, and V. E. Zizler, Separating polynomials on Banach spaces, Quart. J. Math. Oxford Ser. (2) 40 (1989), no. 160, 409-422, DOI 10.1093/qmath/40.4.409. MR.1033216 (91f:46021)

[140] M. Fernández-Unzueta, Zeroes of polynomials on $l_{\infty}$, J. Math. Anal. Appl. 324 (2006), no. 2, 1115-1124, DOI 10.1016/j.jmaa.2006.01.021. MR2266546 (2007g:46068)

[141] J. Ferrer, Zeroes of real polynomials on $C(K)$ spaces, J. Math. Anal. Appl. 336 (2007), no. 2, 788-796, DOI 10.1016/j.jmaa.2007.02.083. MR2352980 (2008m:46092)

[142] J. Ferrer, On the zero-set of real polynomials in non-separable Banach spaces, Publ. Res. Inst. Math. Sci. 43 (2007), no. 3, 685-697. MR2361792(2008h:46019)

[143] J. Ferrer, A note on zeroes of real polynomials in $C(K)$ spaces, Proc. Amer. Math. Soc. 137 (2009), no. 2, 573-577, DOI 10.1090/S0002-9939-08-09574-9. MR2448577 (2010a:46105)

[144] V. P. Fonf, V. I. Gurariy, and M. I. Kadets, An infinite dimensional subspace of C $[0,1]$ consisting of nowhere differentiable functions, C. R. Acad. Bulgare Sci. 52 (1999), no. 1112, 13-16. MR1738120 (2000j:26006)

[145] J. Foran, Fundamentals of real analysis, Monographs and Textbooks in Pure and Applied Mathematics, vol. 144, Marcel Dekker Inc., New York, 1991. MR.1201817 (94e:00002)

[146] D. Gaier, Lectures on complex approximation, Birkhäuser Boston Inc., Boston, MA, 1987. Translated from the German by Renate McLaughlin. MR894920 (88i:30059b)

[147] E. A. Gallardo-Gutiérrez and A. Montes-Rodríguez, The role of the spectrum in the cyclic behavior of composition operators, Memoirs Amer. Math. Soc. 791, AMS, Providence, Rhode Island, 2004.

[148] J. L. Gámez-Merino, Large algebraic structures inside the set of surjective functions, Bull. Belg. Math. Soc. Simon Stevin 18 (2011), no. 2, 297-300. MR2848805 (2012e:26005)

[149] J. L. Gámez-Merino, G. A. Muñoz-Fernández, V. M. Sánchez, and J. B. Seoane-Sepúlveda, Sierpiński-Zygmund functions and other problems on lineability, Proc. Amer. Math. Soc. 138 (2010), no. 11, 3863-3876, DOI 10.1090/S0002-9939-2010-10420-3. MR2679609 (2011g:46042) 
[150] J. L. Gámez-Merino, G. A. Muñoz-Fernández, and J. B. Seoane-Sepúlveda, Lineability and additivity in $\mathbb{R}^{\mathbb{R}}$, J. Math. Anal. Appl. 369 (2010), no. 1, 265-272, DOI 10.1016/j.jmaa.2010.03.036. MR2643865 (2011h:46011)

[151] J. L. Gámez-Merino, G. A. Muñoz-Fernández, and J. B. Seoane-Sepúlveda, A characterization of continuity revisited, Amer. Math. Monthly 118 (2011), no. 2, 167-170, DOI 10.4169/amer.math.monthly.118.02.167. MR2795585 (2012c:26008)

[152] J. L. Gámez-Merino and J. B. Seoane-Sepúlveda, An undecidable case of lineability in $\mathbb{R}^{\mathbb{R}}, \mathrm{J}$. Math. Anal. Appl. 401 (2013), no. 2, 959-962, DOI 10.1016/j.jmaa.2012.10.067. MR3018041

[153] F. J. García-Pacheco, Vector subspaces of the set of non-norm-attaining functionals, Bull. Aust. Math. Soc. 77 (2008), no. 3, 425-432, DOI 10.1017/S0004972708000348. MR2454973 (2009i:46026)

[154] D. García, B. C. Grecu, M. Maestre, and J. B. Seoane-Sepúlveda, Infinite dimensional Banach spaces of functions with nonlinear properties, Math. Nachr. 283 (2010), no. 5, 712720, DOI 10.1002/mana.200610833. MR2666300 (2011e:46041)

[155] F. J. García-Pacheco, M. Martín, and J. B. Seoane-Sepúlveda, Lineability, spaceability, and algebrability of certain subsets of function spaces, Taiwanese J. Math. 13 (2009), no. 4, 1257-1269. MR2543741(2010h:46072)

[156] F. J. García-Pacheco, N. Palmberg, and J. B. Seoane-Sepúlveda, Lineability and algebrability of pathological phenomena in analysis, J. Math. Anal. Appl. 326 (2007), no. 2, 929-939, DOI 10.1016/j.jmaa.2006.03.025. MR2280953 (2007i:26003)

[157] F. J. García-Pacheco, C. Pérez-Eslava, and J. B. Seoane-Sepúlveda, Moduleability, algebraic structures, and nonlinear properties, J. Math. Anal. Appl. 370 (2010), no. 1, 159-167, DOI 10.1016/j.jmaa.2010.05.016. MR2651137(2011h:46012)

[158] F. J. García-Pacheco and D. Puglisi, Lineability of functionals and operators, Studia Math. 201 (2010), no. 1, 37-47, DOI 10.4064/sm201-1-3. MR2733273 (2011j:46010)

[159] F. J. García-Pacheco, F. Rambla-Barreno, and J. B. Seoane-Sepúlveda, Q-linear functions, functions with dense graph, and everywhere surjectivity, Math. Scand. 102 (2008), no. 1, 156-160. MR2420685 (2009c:46002)

[160] F. J. García-Pacheco and J. B. Seoane-Sepúlveda, Vector spaces of non-measurable functions, Acta Math. Sin. (Engl. Ser.) 22 (2006), no. 6, 1805-1808, DOI 10.1007/s10114-0050726-y. MR2262440 (2007i:28006)

[161] B. R. Gelbaum and J. M. H. Olmsted, Counterexamples in analysis, The Mathesis Series, Holden-Day Inc., San Francisco, Calif., 1964. MR0169961 (30 \#204)

[162] B. R. Gelbaum and J. M. H. Olmsted, Theorems and counterexamples in mathematics, Problem Books in Mathematics, Springer-Verlag, New York, 1990. MR1066872 (92b:00003)

[163] B. R. Gelbaum and J. M. H. Olmsted, Counterexamples in analysis, Dover Publications Inc., Mineola, NY, 2003. Corrected reprint of the second (1965) edition. MR 1996162

[164] S. Głąb, P. Kaufmann, and L. Pellegrini, Large structures made of nowhere $L^{p}$ functions, arXiv:1207.3818v1 [math.FA].

[165] S. Głab, P. L. Kaufmann, and L. Pellegrini, Spaceability and algebrability of sets of nowhere integrable functions, Proc. Amer. Math. Soc. 141 (2013), no. 6, 2025-2037, DOI 10.1090/S0002-9939-2012-11574-6. MR3034428

[166] J. Glovebnik, The range of vector-valued analytic functions, Ark. Mat. 14 (1976), 113-118.

[167] G. Godefroy and J. H. Shapiro, Operators with dense, invariant, cyclic vector manifolds, J. Funct. Anal. 98 (1991), no. 2, 229-269, DOI 10.1016/0022-1236(91)90078-J. MR1111569 (92d:47029)

[168] M. Goliński, Invariant subspace problem for classical spaces of functions, J. Funct. Anal. 262 (2012), no. 3, 1251-1273.

[169] M. González, F. León-Saavedra, and A. Montes-Rodríguez, Semi-Fredholm theory: hypercyclic and supercyclic subspaces, Proc. London Math. Soc. (3) 81 (2000), no. 1, 169-189, DOI 10.1112/S0024611500012454. MR.1757050 (2001g:47013)

[170] K. Grandjot, Über Grenzwerte ganzer transzendenter Funktionen, Math. Ann. 91 (1924), no. 3-4, 316-320, DOI 10.1007/BF01556086 (German). MR 1512196

[171] S. Grivaux, Construction of operators with prescribed behaviour, Arch. Math. (Basel) 81 (2003), no. 3, 291-299, DOI 10.1007/s00013-003-0544-3. MR2013260 (2004g:47011)

[172] K.-G. Große-Erdmann, Holomorphe Monster und universelle Funktionen, Mitt. Math. Sem. Giessen 176 (1987), iv+84 (German). Dissertation, University of Trier, Trier, 1987. MR.877464(88i:30060) 
[173] K.-G. Grosse-Erdmann, Universal families and hypercyclic operators, Bull. Amer. Math. Soc. (N.S.) 36 (1999), no. 3, 345-381, DOI 10.1090/S0273-0979-99-00788-0. MR.1685272 (2000c:47001)

[174] K.-G. Grosse-Erdmann, Recent developments in hypercyclicity, RACSAM. Rev. R. Acad. Cienc. Exactas Fís. Nat. Ser. A Mat. 97 (2003), no. 2, 273-286 (English, with English and Spanish summaries). MR2068180 (2005c:47010)

[175] K.-G. Grosse-Erdmann and R. Mortini, Universal functions for composition operators with non-automorphic symbol, J. Anal. Math. 107 (2009), 355-376, DOI 10.1007/s11854-0090013-4. MR2496409 (2010c:30073)

[176] K.-G. Grosse-Erdmann and A. Peris Manguillot, Linear chaos, Universitext, Springer, London, 2011. MR2919812

[177] R. C. Gunning and H. Rossi, Analytic functions of several complex variables, AMS Chelsea Publishing, Providence, RI, 2009. Reprint of the 1965 original. MR2568219 (2010j:32001)

[178] V. I. Gurariŭ, Subspaces and bases in spaces of continuous functions, Dokl. Akad. Nauk SSSR 167 (1966), 971-973 (Russian).

[179] V. I. Gurariǔ, Linear spaces composed of everywhere nondifferentiable functions, C. R. Acad. Bulgare Sci. 44 (1991), no. 5, 13-16 (Russian).

[180] V. I. Gurariy and W. Lusky, Geometry of Müntz spaces and related questions, Lecture Notes in Mathematics, vol. 1870, Springer-Verlag, Berlin, 2005. MR2190706 (2007g:46027)

[181] V. I. Gurariy and L. Quarta, On lineability of sets of continuous functions, J. Math. Anal. Appl. 294 (2004), no. 1, 62-72, DOI 10.1016/j.jmaa.2004.01.036. MR.2059788(2005c:46026)

[182] T. R. Hamlett, Compact maps, connected maps and continuity, J. London Math. Soc. (2) 10 (1975), 25-26. MR0365460 (51 \#1712)

[183] J. Harris, Algebraic geometry, Graduate Texts in Mathematics, vol. 133, Springer-Verlag, New York, 1992. A first course. MR1182558 (93j:14001)

[184] S. Hencl, Isometrical embeddings of separable Banach spaces into the set of nowhere approximatively differentiable and nowhere Hölder functions, Proc. Amer. Math. Soc. 128 (2000), no. 12, 3505-3511, DOI 10.1090/S0002-9939-00-05595-7. MR1707147(2001b:46026)

[185] D. A. Herrero, Limits of hypercyclic and supercyclic operators, J. Funct. Anal. 99 (1991), no. 1, 179-190, DOI 10.1016/0022-1236(91)90058-D. MR.1120920 (92g:47026)

[186] D. Herrero, Hypercyclic operators and chaos, J. Operator Theory 28 (1992), 93-103.

[187] H. M. Hilden and L. J. Wallen, Some cyclic and non-cyclic vectors of certain operators, Indiana Univ. Math. J. 23 (1973/74), 557-565. MR0326452 (48 \#4796)

[188] R. A. Horn, Editor's Endnotes, Amer. Math. Monthly 107 (2000), no. 10, 968-969. MR. 1543790

[189] P. Jiménez-Rodríguez, $c_{0}$ is isometrically isomorphic to a subspace of Cantor-Lebesgue functions, J. Math. Anal. Appl. (to appear).

[190] P. Jiménez-Rodríguez, G. A. Muñoz-Fernández, and J. B. Seoane-Sepúlveda, Non-Lipschitz functions with bounded gradient and related problems, Linear Algebra Appl. 437 (2012), no. 4, 1174-1181, DOI 10.1016/j.laa.2012.04.010. MR2926163

[191] P. Jiménez-Rodríguez, G. A. Muñoz-Fernández, and J. B. Seoane-Sepúlveda, On Weierstrass' Monsters and lineability, Bull. Belg. Math. Soc. Simon Stevin (to appear).

[192] F. B. Jones, Connected and disconnected plane sets and the functional equation $f(x)+f(y)=$ $f(x+y)$, Bull. Amer. Math. Soc. 48 (1942), 115-120. MR0005906 (3,229e)

[193] F. E. Jordan, Cardinal numbers connected with adding Darboux-like functions, ProQuest LLC, Ann Arbor, MI, 1998. Thesis (Ph.D.)-West Virginia University. MR2697132

[194] J. P. Kahane, Baire's category theorem and trigonometric series, J. Analyse Math. 80 (2000), no. 1, 143-182.

[195] Y. Katznelson, An introduction to harmonic analysis, John Wiley \& Sons Inc., New York, 1968. MR0248482(40\#1734)

[196] Y. Katznelson and K. Stromberg, Everywhere differentiable, nowhere monotone, functions, Amer. Math. Monthly 81 (1974), 349-354. MR0335701 (49 \#481)

[197] L. Kaup and B. Kaup, Holomorphic functions of several variables, de Gruyter Studies in Mathematics, vol. 3, Walter de Gruyter \& Co., Berlin, 1983. An introduction to the fundamental theory; With the assistance of Gottfried Barthel; Translated from the German by Michael Bridgland. MR716497 (85k:32001) 
[198] A. B. Kharazishvili, Strange functions in real analysis, 2nd ed., Pure and Applied Mathematics (Boca Raton), vol. 272, Chapman \& Hall/CRC, Boca Raton, FL, 2006. MR2193523 (2006g:26003)

[199] St. Kierst and D. Szpilrajn, Sur certaines singularités des fonctions analytiques uniformes, Fundamenta Math. 21 (1933), 276-294.

[200] S. S. Kim and K. H. Kwon, Smooth $\left(C^{\infty}\right)$ but nowhere analytic functions, Amer. Math. Monthly 107 (2000), no. 3, 264-266, DOI 10.2307/2589322. MR 1742128

[201] D. Kitson and R. M. Timoney, Operator ranges and spaceability, J. Math. Anal. Appl. 378 (2011), no. 2, 680-686, DOI 10.1016/j.jmaa.2010.12.061. MR2773276 (2012b:46018)

[202] S. Kolyada and L. Snoha, Some aspects of topological transitivity - a survey, Iteration theory (ECIT 94) (Opava), Grazer Math. Ber., vol. 334, Karl-Franzens-Univ. Graz, Graz, 1997, pp. 3-35. MR 1644768

[203] T. W. Körner, Fourier analysis, Cambridge University Press, Cambridge, 1988.

[204] S. Koumandos, V. Nestoridis, Y.-S. Smyrlis, and V. Stefanopoulos, Universal series in $\bigcap_{p>1} \ell^{p}$, Bull. Lond. Math. Soc. 42 (2010), no. 1, 119-129, DOI 10.1112/blms/bdp102. MR2586972 (2011b:30139)

[205] K. Kunen, Set theory. An introduction to independence proofs, Studies in Logic and the Foundations of Mathematics, vol. 102, North-Holland Publishing Co., Amsterdam, 1980. MR597342(82f:03001)

[206] H. Lebesgue, Leçons sur l'intégration et la recherche des fonctions primitives, GauthierWillars, 1904.

[207] F. León-Saavedra and A. Montes-Rodríguez, Linear structure of hypercyclic vectors, J. Funct. Anal. 148 (1997), no. 2, 524-545, DOI 10.1006/jfan.1996.3084. MR.1469352 (98h:47028b)

[208] F. León-Saavedra and A. Montes-Rodríguez, Spectral theory and hypercyclic subspaces, Trans. Amer. Math. Soc. 353 (2001), no. 1, 247-267, DOI 10.1090/S0002-9947-00-02743-4. MR $1783790(2001 \mathrm{~h}: 47008)$

[209] F. León-Saavedra and V. Müller, Hypercyclic sequences of operators, Studia Math. 175 (2006), no. 1, 1-18, DOI 10.4064/sm175-1-1. MR2261697 (2007g:47012)

[210] M. Lerch, Ueber die Nichtdifferentiirbarkeit bewisser Funktionen, J. Reine Angew. Math. 103 (1888), 126-138.

[211] B. Levine and D. Milman, On linear sets in space $C$ consisting of functions of bounded variation, Comm. Inst. Sci. Math. Méc. Univ. Kharkoff [Zapiski Inst. Mat. Mech.] (4) 16 (1940), 102-105 (Russian, with English summary). MR0004712 (3,49g)

[212] J. Lindenstrauss, On subspaces of Banach spaces without quasicomplements, Israel J. Math. 6 (1968), 36-38. MR0240599(39 \#1946)

[213] J. López-Salazar Codes, Vector spaces of entire functions of unbounded type, Proc. Amer. Math. Soc. 139 (2011), no. 4, 1347-1360, DOI 10.1090/S0002-9939-2010-10817-1. MR $2748427(2011 \mathrm{~m}: 46069)$

[214] J. López-Salazar, Lineability of the set of holomorphic mappings with dense range, Studia Math. 210 (2012), no. 2, 177-188, DOI 10.4064/sm210-2-5. MR2980006

[215] M. L. Lourenço and N. N. Tocha, Zeros of complex homogeneous polynomials, Linear Multilinear Algebra 55 (2007), no. 5, 463-469, DOI 10.1080/03081080600628273. MR2363547 (2008k:32017)

[216] W. Luh, Approximation analytischer Funktionen durch überkonvergente Potenzreihen und deren Matrix-Transformierten, Mitt. Math. Sem. Giessen Heft 88 (1970), i+56 (German). MR 0280692 (43 \#6411)

[217] W. Luh, Holomorphic monsters, J. Approx. Theory 53 (1988), no. 2, 128-144, DOI 10.1016/0021-9045(88)90060-3. MR.945866 (90a:30091)

[218] G. R. MacLane, Sequences of derivatives and normal families, J. Anal. Math. 2 (1952), no. $1,72-87$.

[219] F. Martínez-Giménez, P. Oprocha, and A. Peris, Distributional chaos for operators with full scrambled sets, Math. Z. (to appear).

[220] Q. Menet, Sous-espaces fermés de séries universelles sur un espace de Fréchet, Studia Math. 207 (2011), no. 2, 181-195, DOI 10.4064/sm207-2-5 (French, with English summary). $\operatorname{MR} 2864388$

[221] Q. Menet, Hypercyclic subspaces on Fréchet spaces without continuous norm, arXiv:1302.6447v1 [math.DS], Preprint (2013). 
[222] Q. Menet, Hypercyclic subspaces and weighted shifts, arXiv:1208.4963v1 [math.DS], Preprint (2013).

[223] A. Montes-Rodríguez, Banach spaces of hypercyclic vectors, Michigan Math. J. 43 (1996), no. 3, 419-436, DOI 10.1307/mmj/1029005536. MR1420585(98g:47027)

[224] A. Montes-Rodríguez, A Birkhoff theorem for Riemann surfaces, Rocky Mountain J. Math. 28 (1998), no. 2, 663-693, DOI 10.1216/rmjm/1181071794. MR/1651593 (99h:30047)

[225] A. Montes-Rodríguez and H. N. Salas, Supercyclic subspaces: spectral theory and weighted shifts, Adv. Math. 163 (2001), no. 1, 74-134, DOI 10.1006/aima.2001.2001. MR.1867204 (2002i:47010)

[226] A. Montes-Rodríguez and H. N. Salas, Supercyclic subspaces, Bull. London Math. Soc. 35 (2003), no. 6, 721-737, DOI 10.1112/S002460930300242X. MR2000019 (2004d:47022)

[227] J. Müller, Continuous functions with universally divergent Fourier series on small subsets of the circle, C. R. Math. Acad. Sci. Paris 348 (2010), no. 21-22, 1155-1158, DOI 10.1016/j.crma.2010.10.026 (English, with English and French summaries). MR2738918 (2011m:42010)

[228] J. Müller, V. Vlachou, and A. Yavrian, Overconvergent series of rational functions and universal Laurent series, J. Anal. Math. 104 (2008), 235-245, DOI 10.1007/s11854-0080023-7. MR2403436 (2009e:30007)

[229] J. Mujica, Complex analysis in Banach spaces, North-Holland Mathematics Studies, vol. 120, North-Holland Publishing Co., Amsterdam, 1986. Holomorphic functions and domains of holomorphy in finite and infinite dimensions; Notas de Matemática [Mathematical Notes], 107. MR842435 (88d:46084)

[230] J. Mujica, Complex analysis in Banach spaces, North-Holland Mathematics Studies, vol. 120, North-Holland Publishing Co., Amsterdam, 1986. Holomorphic functions and domains of holomorphy in finite and infinite dimensions; Notas de Matemática [Mathematical Notes], 107. MR842435(88d:46084)

[231] G. A. Muñoz-Fernández, N. Palmberg, D. Puglisi, and J. B. Seoane-Sepúlveda, Lineability in subsets of measure and function spaces, Linear Algebra Appl. 428 (2008), no. 11-12, 2805-2812, DOI 10.1016/j.laa.2008.01.008. MR2416590 (2009g:46051)

[232] T. Natkaniec, Almost continuity, Real Anal. Exchange 17 (1991/92), no. 2, $462-520$. MR 1171393 (93e:54009)

[233] T. Natkaniec, New cardinal invariants in real analysis, Bull. Polish Acad. Sci. Math. 44 (1996), no. 2, 251-256. MR 1416428(98a:26007)

[234] V. Nestoridis, Universal Taylor series, Ann. Inst. Fourier (Grenoble) 46 (1996), no. 5, 12931306 (English, with English and French summaries). MR1427126 (97k:30001)

[235] J. C. Oxtoby, Measure and category, 2nd ed., Graduate Texts in Mathematics, vol. 2, Springer-Verlag, New York, 1980. A survey of the analogies between topological and measure spaces. MR584443 (81j:28003)

[236] D. Pellegrino and E. V. Teixeira, Norm optimization problem for linear operators in classical Banach spaces, Bull. Braz. Math. Soc. (N.S.) 40 (2009), no. 3, 417-431, DOI 10.1007/s00574009-0019-7. MR2540517 (2010i:47066)

[237] H. Petersson, Hypercyclic subspaces for Fréchet space operators, J. Math. Anal. Appl. 319 (2006), no. 2, 764-782, DOI 10.1016/j.jmaa.2005.06.042. MR2227937(2007b:47019)

[238] A. Plichko and A. Zagorodnyuk, On automatic continuity and three problems of The Scottish book concerning the boundedness of polynomial functionals, J. Math. Anal. Appl. 220 (1998), no. 2, 477-494, DOI 10.1006/jmaa.1997.5826. MR1614947(99c:46050)

[239] K. Płotka, Sum of Sierpiński-Zygmund and Darboux like functions, Topology Appl. 122 (2002), no. 3, 547-564, DOI 10.1016/S0166-8641(01)00184-5. MR1911699(2003e:26002)

[240] E. E. Posey and J. E. Vaughan, Functions with a proper local maximum in each interval, Amer. Math. Monthly 90 (1983), no. 4, 281-282, DOI 10.2307/2975762. MR700268 (85a:26012)

[241] A. Pringsheim, Ueber die Multiplication bedingt convergenter Reihen, Math. Ann. 21 (1883), no. 3, 327-378, DOI 10.1007/BF01443879 (German). MR 1510201

[242] D. Puglisi and J. B. Seoane-Sepúlveda, Bounded linear non-absolutely summing operators, J. Math. Anal. Appl. 338 (2008), no. 1, 292-298, DOI 10.1016/j.jmaa.2007.05.029. MR2386416 (2009e:47038) 
[243] C. J. Read, The invariant subspace problem for a class of Banach spaces. II. Hypercyclic operators, Israel J. Math. 63 (1988), no. 1, 1-40, DOI 10.1007/BF02765019. MR959046 (90b:47013)

[244] D. A. Redett, Strongly annular functions in Bergman space, Comput. Methods Funct. Theory 7 (2007), no. 2, 429-432. MR2376682 (2008j:46020)

[245] L. Rodríguez-Piazza, Every separable Banach space is isometric to a space of continuous nowhere differentiable functions, Proc. Amer. Math. Soc. 123 (1995), no. 12, 3649-3654, DOI 10.2307/2161889. MR1328375 (96d:46007)

[246] S. Rolewicz, On orbits of elements, Studia Math. 32 (1969), 17-22.

[247] H. P. Rosenthal, On quasi-complemented subspaces of Banach spaces, Proc. Nat. Acad. Sci. U.S.A. 59 (1968), 361-364. MR0227739 (37 \#3323)

[248] W. Rudin, Holomorphic maps of discs into F-spaces, Complex analysis (Proc. Conf., Univ. Kentucky, Lexington, Ky., 1976), Springer, Berlin, 1977, pp. 104-108. Lecture Notes in Math., Vol. 599. MR0499229 (58 \#17142)

[249] W. Rudin, Real and complex analysis, 3rd ed., McGraw-Hill Book Co., New York, 1987. MR.924157 (88k:00002)

[250] W. Rudin, Functional analysis, 2nd ed., International Series in Pure and Applied Mathematics, McGraw-Hill Inc., New York, 1991. MR.1157815 (92k:46001)

[251] H. N. Salas, Supercyclicity and weighted shifts, Studia Math. 135 (1999), no. 1, 55-74. MR 1686371 (2000b:47020)

[252] H. Salzmann and K. Zeller, Singularitäten unendlich oft differenzierbarer Funktionen, Math. Z. 62 (1955), 354-367 (German). MR0071479 (17,134b)

[253] J. B. Seoane-Sepúlveda, Chaos and lineability of pathological phenomena in analysis, ProQuest LLC, Ann Arbor, MI, 2006. Thesis (Ph.D.)-Kent State University. MR2709064

[254] J. B. Seoane-Sepúlveda, Explicit constructions of dense common hypercyclic subspaces, Publ. Res. Inst. Math. Sci. 43 (2007), no. 2, 373-384. MR2341015 (2008g:47021)

[255] J. H. Shapiro, Composition operators and classical function theory, Universitext: Tracts in Mathematics, Springer-Verlag, New York, 1993. MR.1237406 (94k:47049)

[256] B. Schweizer and J. Smítal, Measures of chaos and a spectral decomposition of dynamical systems on the interval, Trans. Amer. Math. Soc. 344 (1994), no. 2, 737-754, DOI 10.2307/2154504. MR1227094 (94k:58091)

[257] J. Shinoda, Some consequences of Martin's axiom and the negation of the continuum hypothesis, Nagoya Math. J. 49 (1973), 117-125. MR0319754(47 \#8296)

[258] S. Shkarin, On the set of hypercyclic vectors for the differentiation operator, Israel J. Math. 180 (2010), 271-283, DOI 10.1007/s11856-010-0104-z. MR2735066 (2012b:47022)

[259] S. Shkarin, Hypercyclic operators on topological vector spaces, J. London Math. Soc. (2) 86 (2012), no. 1, 195-213.

[260] J. Siciak, Highly noncontinuable functions on polynomially convex sets, Complex analysis (Toulouse, 1983), Lecture Notes in Math., vol. 1094, Springer, Berlin, 1984, pp. 173-178, DOI 10.1007/BFb0099161. MR773109 (86e:32009)

[261] W. Sierpiński and A. Zygmund, Sur une fonction qui est discontinue sur tout ensemble de puissance du continu, Fund. Math. 4 (1923), 316-318.

[262] J. Stallings, Fixed point theorems for connectivity maps, Fund. Math. 47 (1959), 249-263. MR0117710 (22 \#8485)

[263] L. A. Steen and J. A. Seebach Jr., Counterexamples in topology, 2nd ed., Springer-Verlag, New York, 1978. MR507446 (80a:54001)

[264] J. Thim, Continuous nowhere differentiable functions, Luleå University of Technology, 2003. Masters Thesis.

[265] S. Todorčević, Partitioning pairs of countable ordinals, Acta Math. 159 (1987), no. 3-4, 261-294, DOI 10.1007/BF02392561. MR908147 (88i:04002)

[266] M. Valdivia, The space $\mathcal{H}\left(\Omega,\left(z_{j}\right)\right)$ of holomorphic functions, J. Math. Anal. Appl. 337 (2008), no. 2, 821-839.

[267] M. Valdivia, Spaces of holomorphic functions in regular domains, J. Math. Anal. Appl. 350 (2009), no. 2, 651-662.

[268] D. J. Velleman, Characterizing continuity, Amer. Math. Monthly 104 (1997), no. 4, 318-322, DOI 10.2307/2974580. MR 1450665 (98e:26002)

[269] C. E. Weil, On properties of derivatives, Trans. Amer. Math. Soc. 114 (1965), 363-376. MR0176007 (31 \#283) 
[270] J. Wengenroth, Hypercyclic operators on non-locally convex spaces, Proc. Amer. Math. Soc. 131 (2003), no. 6, 1759-1761 (electronic), DOI 10.1090/S0002-9939-03-07003-5. MR.1955262 (2003j:47007)

[271] D. J. White, Functions preserving compactness and connectedness are continuous, J. London Math. Soc. 43 (1968), 714-716. MR0229211 (37 \#4785)

[272] A. Wilansky, Semi-Fredholm maps in FK spaces, Math. Z. 144 (1975), 9-12.

[273] G. L. Wise and E. B. Hall, Counterexamples in probability and real analysis, The Clarendon Press Oxford University Press, New York, 1993. MR.1256489 (95c:60002)

[274] Z. Zahorski, Supplément au mémoire "Sur l'ensemble des points singuliers d'une fonction d'une variable réelle admettant les dérivées de tous les order", Fund. Math. 36 (1949), 319-320 (French). MR0035329 (11,718a)

[275] L. Zalcman, Uniqueness and nonuniqueness for the Radon transform, Bull. London Math. Soc. 14 (1982), no. 3, 241-245, DOI 10.1112/blms/14.3.241. MR656606 (83h:42020)

Departamento de Análisis Matemático, Facultad de Matemáticas, Universidad de Sevilla, Apdo. 1160, Avenida Reina Mercedes, Sevilla, 41080, Spain

E-mail address: lbernal@us.es

Departamento de Matemática, Universidade Federal da Paraíba, 58.051-900 - João PESSOA, BraziL

E-mail address: pellegrino@pq.cnpq.br; dmpellegrino@gmail.com

Departamento de Análisis Matemático, Facultad de Ciencias Matemáticas, Plaza de Ciencias 3, Universidad Complutense de Madrid, Madrid, 28040, Spain

E-mail address: jseoane@mat.ucm.es 